Pontifícia Universidade $C_{\text {atólica }}$

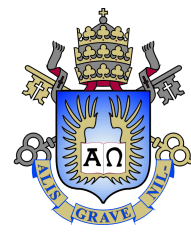

Renan Vieira Bela

\title{
Pressure Transient Analysis for Injectivity Tests in Multilayer Reservoirs
}

Tese de Doutorado

Thesis presented to the Programa de Pós-graduação em Matemática, do Departamento de Matemática da PUC-Rio in partial fulfillment of the requirements for the degree of Doutor em Matemática.

Advisor

Prof. Sinesio Pesco

Co-advisor: Prof. Abelardo Borges Barreto Jr. 


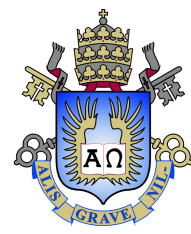

Renan Vieira Bela

\section{Pressure Transient Analysis for Injectivity Tests in Multilayer Reservoirs}

Thesis presented to the Programa de Pós-graduação em Matemática da PUC-Rio in partial fulfillment of the requirements for the degree of Doutor em Matemática. Approved by the Examination Committee:

Prof. Sinesio Pesco

Advisor

Departamento de Matemática - PUC-Rio

Prof. Abelardo Borges Barreto Jr.

Co-advisor

Departamento de Engenharia Mecânica - PUC-Rio

Prof. Adolfo Puime Pires

Laboratório de Engenharia e Exploração de Petróleo - LENEP -

UENF

Prof. Boyan Slavchev Sirakov

Departamento de Matemática - PUC-Rio

Prof. Marcio da Silveira Carvalho

Departamento de Engenharia Mecânica - PUC-Rio

Prof. Mustafa Onur

McDougall School of Petroleum Engineering - The University of

Tulsa - TU

Rio de Janeiro, December the 3rd, 2021 
All rights reserved.

\section{Renan Vieira Bela}

Holds a bachelor major degree in Chemical Engineering and minor degree in Mathematical Studies, both at PUC-Rio. Master of Science in Applied Mathematics at PUC-Rio.

Bibliographic data

Bela, Renan Vieira

Pressure Transient Analysis for Injectivity Tests in Multilayer Reservoirs / Renan Vieira Bela; advisor: Sinesio Pesco; co-advisor: Abelardo Borges Barreto Jr.. - 2021.

83 f: il. color. ; $30 \mathrm{~cm}$

Tese (doutorado) - Pontifícia Universidade Católica do Rio de Janeiro, Departamento de Matemática, 2021.

Inclui bibliografia

1. Matemática - Teses. 2. Testes de Injetividade. 3. Análise de Transiente de Pressão. 4. Reservatórios Estratificados. 5. Formulação Analítica. 6. Funções de Green. I. Pesco, Sinesio. II. Barreto Jr., Abelardo Borges. III. Pontifícia Universidade Católica do Rio de Janeiro. Departamento de Matemática. IV. Título. 


\section{Acknowledgments}

First I would like to thank my advisor Sinesio and my co-advisor Abelardo for the opportunity, for believing that I could deliver a decent $\mathrm{PhD}$ thesis and for the lessons that go way beyond the technical skills.

To my colleagues and co-workers who somehow contributed to the development of this work, specially prof Onur, who helped me to grow as researcher despite the little time I spent in the University of Tulsa. To my friend Thiago, who consistently improved my work with the many tips, suggestions and discussions.

To my parents and my siblings and to my grandmother, that supported me every time.

I would also like to thank PUC-Rio, a place that has been my second home for the last 11 years, as well as every professor, employee or staff member that assisted me along this journey (and who are too many to be individually mentioned).

I am grateful to Petrobras for partially sponsoring this research as part of the project TCBR 485 .

This study was financed in part by the Coordenação de Aperfeiçoamento de Pessoal de Nível Superior - Brasil (CAPES) - Finance Code 001. 


\section{Abstract}

Bela, Renan Vieira; Pesco, Sinesio (Advisor); Barreto Jr., Abelardo Borges (Co-Advisor). Pressure Transient Analysis for Injectivity Tests in Multilayer Reservoirs. Rio de Janeiro, 2021. 83p. Tese de Doutorado - Departamento de Matemática, Pontifícia Universidade Católica do Rio de Janeiro.

Analytical models that describe the pressure behavior are extremely useful for pressure transient analysis and reservoir characterization as they provide estimates of reservoir parameters. This work has two main goals: first, to extend the existing solutions for injectivity/falloff tests in single-layer formations with horizontal wells so that they can be applied to multilayer stratified reservoirs with multilateral horizontal wells. Furthermore, this work applies impulse functions to obtain an alternative formulation for injectivity tests in multilayer commingled formations with vertical wells and single-layer reservoirs with horizontal wells.

\section{Keywords}

Injectivity Tests; Pressure Transient Analysis; Stratified Reservoirs; Analytical Modeling; Green's Functions. 


\section{Resumo}

Bela, Renan Vieira; Pesco, Sinesio; Barreto Jr., Abelardo Borges. Análise dos Dados Transientes de Pressão durante Testes de Injetividade em Reservatórios Multicamadas. Rio de Janeiro, 2021. 83p. Tese de Doutorado - Departamento de Matemática, Pontifícia Universidade Católica do Rio de Janeiro.

Modelos analíticos que descrevam o comportamento da pressão são de extrema utilidade na área de avaliação de formações e caracterização de reservatório, pois eles fornecem estimativas sobre diversos parâmetros do reservatório. Este trabalho tem dois objetivos principais: primeiro, estender a solução existente para testes de injetividade e falloff em reservatórios com uma camada e poços horizontais de modo que ela possa ser aplicada também em formações multicamadas com poços horizontais multirramificados. Além disso, este trabalho aplica funções impulso para obter uma formulação alternativa para testes de injetividade em reservatórios estratificados com poços verticais e formações com uma camada e poços horizontais.

\section{Palavras-chave}

Testes de Injetividade; Análise de Transiente de Pressão; Reservatórios Estratificados; Formulação Analítica; Funções de Green. 


\section{Table of contents}

1 Introduction $\quad 13$

2 Analytical Solution for Injectivity and Falloff Tests in Stratified Reservoirs with Multilateral Horizontal Wells $\quad 16$

2.1 Introduction $\quad 16$

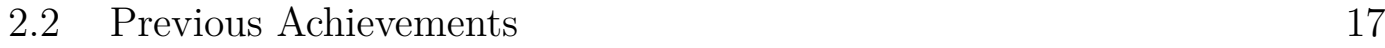

2.2.1 Formulations for Single-Phase Flow in Single-Lateral Wells 17

2.2.2 Formulations for Single-Phase Flow in Multilateral Wells 20

2.2.3 Formulations for Injectivity Tests in Horizontal Wells 21

2.3 Injectivity Tests with Multilateral Horizontal Wells 22

2.3.1 Injectivity Tests in Single-Layer Reservoirs 23

2.3.2 Injectivity Tests in Multilayer Reservoirs 25

$\begin{array}{lll}2.3 .3 & \text { Formulation for Falloff Tests } & 29\end{array}$

2.3.4 Pressure Derivative Behavior 32

2.3.5 Discussion on the Main Assumptions 34

$\begin{array}{lll}2.4 & \text { Model Validation } & 36\end{array}$

2.5 Summary and Conclusions 46

3 Impulse Functions Applied to Compute Pressure Change During Injectivity Tests 48

$\begin{array}{lll}3.1 & \text { Introduction } & 48\end{array}$

3.2 Proposed Formulation $\quad 51$

3.2.1 Impulse Function for Single-Layer Reservoirs with Vertical Wells 51

3.2.2 Extension to Multilayer Reservoirs 56

3.2.3 Application to Single-Layer Reservoirs with Horizontal Wells 58

$\begin{array}{lll}3.3 & \text { Results and Discussion } & 60\end{array}$

3.3.1 Results for Multilayer Reservoirs with Vertical Wells 61

3.3.2 Results for Single-Layer Reservoirs with Horizontal Wells 67

$\begin{array}{lll}3.4 & \text { Summary and Conclusions } & 70\end{array}$

4 Summary and Suggestions for Future Work $\quad 71$

$\begin{array}{ll}\text { Bibliography } & 73\end{array}$

A Single-Phase Flow Through Multilateral Horizontal Wells 79

A.1 Single-Phase Flow in Single-Layer Reservoirs 79

A.2 Single-Phase Flow in Multilayer Reservoirs 80

B Sketch Proof to Justify the Application of Newman's Product 82 


\section{List of figures}

Figure 1.1 General pressure behavior during a conventional well testing

Figure 1.2 General pressure behavior during an injectivity test

Figure 2.1 Multilayer reservoir model with a multilateral horizontal wellbore

Figure 2.2 Linear flood front propagation from a non centered horizontal well

$\begin{array}{lll}\text { Figure 2.3 } & \text { Relative permeability curves } & 37\end{array}$

Figure 2.4 Pressure data for Case A during injection 38

Figure 2.5 Pressure data for Case A during falloff 38

Figure 2.6 Comparison to single-phase responses for Case A during injection

Figure 2.7 Comparison to single-phase responses for Case A during falloff

Figure 2.8 Pressure data for Case B during injection

Figure 2.9 Pressure data for Case B during falloff

Figure 2.10 Comparison to single-phase responses for Case B during injection

Figure 2.11 Comparison to single-phase responses for Case B during falloff

Figure 2.12 Pressure data for Case C during injection 44

Figure 2.13 Pressure data for Case C during falloff

Figure 2.14 Comparison to single-phase responses for Case C during injection

Figure 2.15 Comparison to single-phase responses for Case C during falloff

Figure 3.1 Schematics of rate transient front and water saturation front in a single-layer reservoir

Figure 3.2 Layer flow-rate profiles at the wellbore (on the left) and rate and saturation fronts in each layer (on the right)

Figure 3.3 Reservoir top (on the left) and lateral views for $r_{F}<r_{\text {skin }} .51$

Figure 3.4 Reservoir top (on the left) and lateral views for $r_{F}>r_{\text {skin }} .52$

Figure 3.5 Schematics of a multilayer reservoir with vertical injector well

Figure 3.6 Flowchart to implement the proposed formulation $\quad 57$

Figure 3.7 Horizontal injector well model (lateral view) 58

Figure 3.8 Horizontal injector well model (view of the $z x$-plane) 59

$\begin{array}{lll}\text { Figure 3.9 Relative permeability data } & 61\end{array}$

Figure 3.10 Pressure and pressure derivative curves for Case A 62

Figure 3.11 Layer flow-rate profiles for Case A (layer 1 in black; layer 2 in blue; layer 3 in red)

Figure 3.12 Pressure and pressure derivative curves for Case B 64 
Figure 3.13 Layer flow-rate profiles for Case B (layer 1 in black; layer 2 in blue; layer 3 in red)

Figure 3.14 Pressure and pressure derivative curves for case $\mathrm{C}$

Figure 3.15 Layer flow-rate profiles for case C (layer 1 in black; layer 2 in blue)

Figure 3.16 Pressure and pressure derivative curves for case D

65

Figure 3.17 Pressure and pressure derivative curves for case E

68

69

Figure B.1 Schematics of the one-dimensional flow in $y$-direction 82

Figure B.2 Schematics of the one-dimensional flow in $r$-direction 


\section{List of tables}

$\begin{array}{lll}\text { Table 2.1 } & \text { Reservoir parameters } & 37\end{array}$

Table 2.2 Estimated equivalent permeabilities 46

Table 3.1 Reservoir properties for the multilayer cases 61

Table 3.2 Estimated layer permeabilities (values in $\mathrm{mD}$, errors in percentage) 67

Table 3.3 Reservoir properties for the cases with horizontal well 68 


\section{List of Symbols}

$A=$ Weighting variable for the injection period, defined in Eq. (2-11)

$a=$ Multiplicative coefficient in Eq. (3-10)

$b=$ Multiplicative coefficient in Eq. (3-10)

$C=$ Wellbore storage coefficient, $\left[\mathrm{m}^{3} / \mathrm{Pa}, \mathrm{bbl} / \mathrm{psi}, \mathrm{m}^{3} /\left(\mathrm{kgf} / \mathrm{cm}^{2}\right)\right]$

$c=$ Compressibility, $\left[(\mathrm{Pa})^{-1},(\mathrm{psia})^{-1}\left(\mathrm{kgf} / \mathrm{cm}^{2}\right)^{-1}\right]$

$d z=$ Smallest distance between the wellbore and a vertical boundary, $[\mathrm{m}, \mathrm{ft}]$

erf $=$ Error function

$\exp =$ Exponential function

$f_{w}^{\prime}=$ Fractional flow derivative [dimensionless]

$G=$ Impulse function

$h=$ Thickness, $[\mathrm{m}, \mathrm{ft}]$

$I_{i}=$ Modified Bessel's function of first kind and order $i$

$K_{i}=$ Modified Bessel's function of second kind and order $i$

$k=$ Permeability, $\left[\mathrm{m}^{2}, \mathrm{mD}\right]$

$k_{r f}=$ Relative permeability to phase $f$, [dimensionless]

$L=$ Wellbore length, $[\mathrm{m}, \mathrm{ft}]$

$\ln =$ Natural logarithm function

$M=$ Matrix defined in Eq. 3-17 $\hat{M}=$ Endpoint mobility ratio, [dimensionless]

$n=$ Total number of layers, [dimensionless]

$p=$ Pressure, $\left[\mathrm{Pa}, \mathrm{psi}, \mathrm{kgf} / \mathrm{cm}^{2}\right]$

$q=$ Flow-rate, $\left[\mathrm{m}^{3} / \mathrm{s}, \mathrm{STB} / \mathrm{d}, \mathrm{m}^{3} / \mathrm{d}\right]$

$R=$ Weighting variable for the falloff period, defined in Eq. (2-24)

$r=$ Radius, $[\mathrm{m}, \mathrm{ft}]$

$r_{w}=$ Wellbore radius, $[\mathrm{m}, \mathrm{ft}]$

$S=$ Skin factor, [dimensionless] 
$S_{w}=$ Water saturation, [dimensionless]

$\sin =$ Sine function

$t=$ Time, $[\mathrm{s}, \mathrm{h}]$

$u=$ Laplace variable

$\beta=$ Upper integral limit in Eqs. (2-1) and (2-7), defined in Eq. (2-2) [m, ft]

$\eta=$ Hydraulic diffusivity coefficient $\left[\mathrm{m}^{2} / \mathrm{s}, \mathrm{mD} \cdot \mathrm{kgf} / \mathrm{cm}^{2} / \mathrm{cP}\right]$

$\gamma=$ Multiplicative constant defined in Eq. (3-18), [dimensionless]

$\mathcal{L}=$ Laplace transform operator

$\mathcal{L}^{-1}=$ Inverse Laplace transform operator

$\lambda=$ Fluid mobility, $\left[(\mathrm{Pa} \cdot \mathrm{s})^{-1},(\mathrm{cP})^{-1}\right]$

$\mu=$ Viscosity, $[\mathrm{Pa} \cdot \mathrm{s}, \mathrm{cP}]$

$\phi=$ Porosity [dimensionless]

$\tau=$ Silent time integration variable $[\mathrm{s}, \mathrm{h}]$

\section{Subscripts}

$e q=$ Equivalent

$F=$ Related to the flood front

$i=$ Related to region $i$

$i n j=$ Related to the injected fluid

$j=$ Related to layer $j$

$o=$ Related to oil

skin $=$ Related to the damaged region

$t=$ Total

$x=$ With respect to $x$-direction

$x y=$ With respect to $x y$-plane

$y=$ With respect to $y$-direction

$z=$ With respect to $z$-direction

$z x=$ With respect to $z x$-plane 


\section{Introduction}

Pressure transient analysis is crucial in reservoir engineering, as it provides valuable estimates about reservoir features and parameters. Adequate reservoir resources management relies on knowledge regarding its parameters, such as permeability and extension.

One conventional way to estimate those parameters consists of well testing, during which fluid is removed from the reservoir and pressure data are measured. At a first stage, the wellbore is open, fluid is produced (preferably at a specified constant production rate) and, thus, pressure decreases. Afterwards, the wellbore is shut in and pressure rises [1, 2, 3, 4]. Those flow periods are called drawdown and buildup, respectively [2, 5, 6]. Fig. 1.1 portrays a qualitative representation of the pressure behavior that is observed during a conventional well testing.

Injectivity tests are an alternative to conventional well testing $[1,7,8]$. This procedure is based on pressure transient analysis due to the injection of an external fluid into the reservoir [9, 10]. Injectivity tests have gained more attention recently, as they are operationally safer and avoid dealing with the produced fluid disposal or flaring - hence, presenting lower environmental impact than conventional well testing $[7,8]$. The reduced carbon footprint compared to conventional well testing becomes particularly important considering that many oil field companies committed to substantially decrease greenhouse gas emissions in a foreseeable future.

Analogously to well testing, two distinct stages are also observed at an injectivity test: injection and falloff. During the former, a fluid is injected into the reservoir and pressure rises. The latter is identified by a pressure drop, after the well is shut $[11,12]$. In Fig. 1.2, a qualitative representation of the pressure behavior measured during an injectivity test may be found.

Although flow simulators may be employed to model multiphase flow, the numerical simulation may be computationally expensive. Moreover, finding appropriate spatial gridding schemes and time step sizes to obtain accurate pressure transient data under such conditions is not an easy task. Besides, flow simulators may not be easily coupled with nonlinear regression methods.

To get around those setbacks, analytical models that accurately describe 


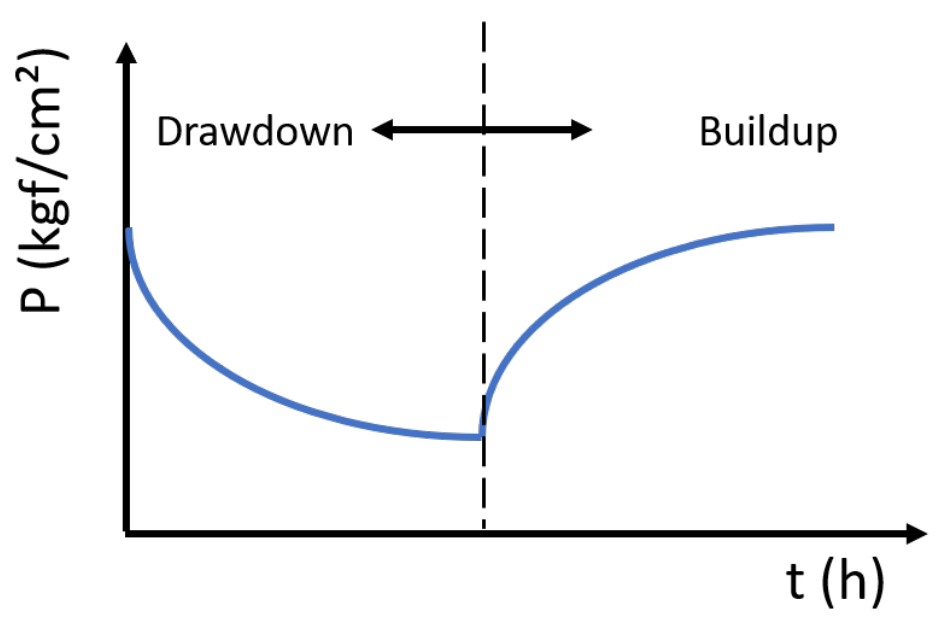

Figure 1.1: General pressure behavior during a conventional well testing

the pressure behavior may be employed to obtain relevant insights regarding reservoir features and estimate its parameters. Besides, analytical solutions are effortlessly coupled with nonlinear regression techniques and optimization methods $[13,14]$.

The Thompson and Reynolds' [15] steady-state theory has been used as basis for the development of analytical solutions for injectivity tests in single$[9,11]$ and multilayer reservoirs $[12,16]$ with vertical wells. In reservoirs with horizontal wells, on the other hand, currently existing analytical solutions can only describe the pressure behavior in single-layer reservoirs $[10,11,17]$.

Furthermore, formulations based on the Thompson and Reynolds' [15] theory consider that the rate transient front is ahead of the water saturation front $[9,16]$. This assumption is valid for most practical cases in single-layer formations [10]. Nonetheless, in multilayer reservoirs, layer flow-rates at the

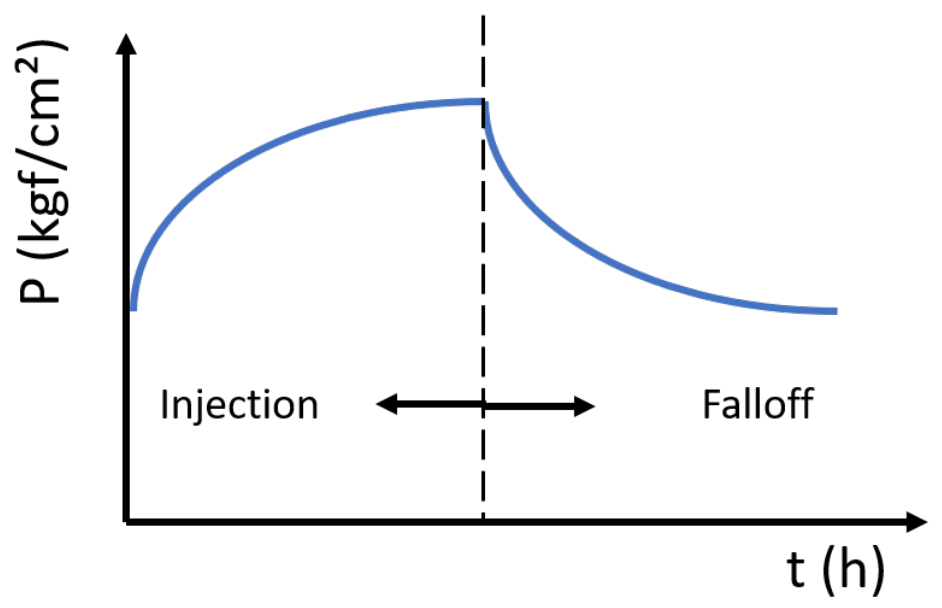

Figure 1.2: General pressure behavior during an injectivity test 
wellbore may change in time $[14,16]$. Thus, in a strict sense, the Thompson and Reynolds' [15] steady-state theory is not applicable for multilayer systems, as the rate transient at the wellbore implies that flow-rate within the flooded region is not necessarily constant.

Therefore, this work has two main goals: first, a formulation for injection/falloff tests in multilayer reservoirs with multilateral horizontal wells is proposed. This model, which is presented in Chapter 2, is based on the existing single-layer solutions $[10,11,17]$. Next, in Chapter 3, Green's functions are employed to develop an alternative formulation for injectivity tests in multilayer reservoirs with vertical wells. An application of Green's functions to obtain the pressure response in single-layer reservoirs with horizontal wells is also presented. Finally, Chapter 4 contains the main conclusions of this work. 


\section{Analytical Solution for Injectivity and Falloff Tests in Strati- fied Reservoirs with Multilateral Horizontal Wells}

This Chapter introduces the proposed model for injection/falloff tests in multilayer reservoirs with multilateral horizontal wells. The formulation is based on the solutions for single-layer reservoirs developed by Peres and Reynolds [10] and Peres et al. [11].

This Chapter is organized as follows: Section 2.1 presents the motivation to obtain a model for injection/falloff tests in multilayer reservoirs with multilateral wells. An overview about previous works regarding analytical solutions for horizontal wells is given in Section 2.2. Section 2.3 details the proposed analytical solutions. The results and discussion are presented in Section 2.4. Lastly, the main conclusions of this Chapter are reported in Section 2.5 .

\section{1 \\ Introduction}

Injectivity tests may be performed either in vertical or horizontal wells. Horizontal wells present some advantages compared to vertical wells, such as reduced fluid coning and better performance in reservoirs with high vertical permeability [18]. Moreover, horizontal wells also enable drilling operations underneath a permanent structure (an airport or ecologically sensitive areas, for instance) and a single horizontal wellbore can replace several vertical wells [19].

The solutions for single-phase $[3,5,20]$ and two-phase flow $[8,11,12,16]$ in multilayer reservoirs with vertical wells are already known. Formulations for well testing in single-layer reservoirs with horizontal wells are also available $[4,18,21,22,23,24,25,26]$. Moreover, analytical models for drawdown and buildup considering multilateral horizontal wells in single-layer [27, 28] and multilayer formations $[29,30,31]$ were developed. However, the currently available solutions for injectivity and falloff tests with horizontal wells are limited to single-layer reservoirs with single-lateral wells [10, 11]. Section 2.2 provides a more detailed literature review regarding analytical models for horizontal wells. 
This Chapter presents the extension of the analytical solutions developed by Peres and Reynolds [10] and Peres et al. [11] for injectivity tests in single-layer reservoirs so that they can be applied in multilayer reservoirs with multilateral horizontal wellbores. It should be noted that models for predicting pressure transient responses during the injection and falloff periods in multilayer reservoirs with a vertical well have already been developed $[12,16,32]$.

In this Chapter, a new model is proposed for predicting pressure transient responses during injection and falloff tests for multilateral horizontal wells completed in multilayer commingled systems. The proposed solutions were validated via comparison with a general purpose finite difference-based commercial flow simulator. Besides, approximations for early- and late-time pressure derivative are provided. These approximations for pressure derivative can be used to estimate the reservoir equivalent permeability. These solutions could be useful for interpreting and analyzing injectivity pressure data sets by nonlinear regression and designing such tests.

\section{2}

\section{Previous Achievements}

This Section reviews the works that provided relevant improvements in understanding flow through horizontal wellbores. As disclosed by this literature review, the currently available models are unable to describe injectivity tests in multilayer systems.

\subsection{1}

\section{Formulations for Single-Phase Flow in Single-Lateral Wells}

The first analytical models for single-phase flow through horizontal wellbores were presented in the late 80's. Goode and Thambynayagam [4] presented an analytical solution for pressure behavior through horizontal wells by applying successive Laplace and Fourier transforms. They also proposed approximate expressions for each observed flow regime. Their formulation uses the superposition principle to compute buildup pressure.

Daviau et al. [21] proposed a solution for single-layer reservoirs based on Green's functions and the sources/sinks method. Their formulation assumes a uniform flux wellbore condition, that is, flux along the wellbore is constant. They also made brief comments regarding the different flow regimes observed and the influence of wellbore hydraulics and positioning on pressure response.

Following their work, Ozkan et al. [18] provided further insights over the existence of two distinct radial flow regimes that may be detected at well 
testing with horizontal wells. Anisotropy was included and they suggested a technique to estimate the reservoir permeability and skin factor from the pressure derivative with respect to the natural logarithm of time (hereafter referred to as pressure derivative). Besides, it was stated that the pressure response at a wellbore with infinite conductivity may be estimated by the equivalent pressure point of an analytical model that assumes uniform flux.

An analytical model for the infinite conductivity wellbore was developed by Rosa and Carvalho [33]. Their hypothesis considers that pressure (rather than fluid influx) in the wellbore interior is uniform. To solve the problem, they proposed that the well may be divided into $n$ segments, each of them presenting uniform influx. Pressure equality in all segments, combined with the constant total producing flow-rate, yields a linear system that allows the determination of flow-rate in each segment.

Abbaszadeh and Hegeman [34] presented a more general solution for slanted wells in reservoirs with a pressure support system. Their formulation accounts for no-flow vertical boundaries, constant pressure vertical boundaries and mixed boundaries. The wellbore is described as a source strip, and pressure change is obtained using Green's functions. Moreover, vertical and horizontal wells may be understood as limiting cases of their solution, by setting the wellbore inclination angle as $0^{\circ}$ and $90^{\circ}$, respectively. They applied the average pressure technique to obtain the pressure response from an infinite conductivity well.

Approximations for each flow period and the required conditions for them to develop were summarized by Odeh and Babu [6], as well as estimates for their starting and ending times. Once again, buildup pressure is computed through the superposition principle.

The solution for horizontal wells in Laplace domain was presented by Kuchuk et al. [22]. Their model is flexible when it comes to the reservoir vertical boundary condition, since it is applicable in cases with constant pressure maintenance systems, such as gas caps, acting at one of the reservoir vertical boundaries.

Jelmert and Thompson [35] proposed that the linear system associated with the infinite conductivity condition is easier to compute in Laplace domain than in the real field. They studied the influence of assuming a piecewise linear flow-rate distribution, when compared to a stepwise constant flow-rate distribution, as suggested by Rosa and Carvalho [33]. Their results supported that it is possible to apply the equivalent pressure point of an uniform influx model to compute the pressure response of an infinite conductivity well.

Spivey and Lee [36] presented a model for wells at an arbitrary azimuth 
in anisotropic formations. Horizontal wells are represented in their model as limiting cases where the deviation angle is zero. The proposed formulation consists of transforming the anisotropic system into an equivalent isotropic reservoir. The model is also applicable at hydraulically fractured wells.

A generalized solution for slanted wells was reached by Zhan and Zlotnik [19]. Their solution consists of dividing the wellbore into several sink points. Then, the solution for those points is determined in Laplace domain. Horizontal and vertical wells may be expressed as particular cases of their formulation.

Nie et al. [24] provided an analytical model for single-phase flow in radially composite single-layer reservoirs with horizontal wells. Their formulation is flexible when it comes to the reservoir boundary conditions: vertical boundaries may be closed or at constant pressure, as well as the lateral boundaries. Type curves were developed considering this composite reservoir scheme. They also showed how pressure derivative gets progressively influenced by the radial zones further from the wellbore as time goes on.

Both the infinite conductivity model and the uniform flux assumption fail to adequately represent the pressure drop inside the wellbore. This pressure change must occur so that fluid moves the well toe to the heel (or from heel to toe, in the case of fluid injection). Hence, a more thorough model should account for frictional, fluid acceleration and directional flow effects. In slanted wells, gravitational effects should also be considered. Chen et al. [25] presented a finite conductivity solution by including the first and second factors above mentioned, considering a multiple-fractured horizontal well. Their results show that the relevance of wellbore hydraulics on pressure change increases until a peak and then declines.

Wang and Zhan [26] have also evaluated the influence of wellbore hydraulics into the pressure response. They compared the equipotential curves in a given reservoir assuming three distinct wellbore conditions: uniform influx, infinite conductivity and mixed inner boundary condition, which describes the wellbore hydraulics in a more realistic way. They showed that, even though pressure change behaves as a linear function of flow-rate for both uniform influx and infinite conductivity, the same does not hold for a mixed boundary condition.

A formulation in Laplace domain for a single-lateral well in a multilayer reservoir with crossflow was developed by Kuchuk and Habashy [23]. Their solution assumes that the wellbore perforates only one layer and, as the test goes on, pressure behavior gets progressively influenced by the other layers, due to formation crossflow. In stratified reservoirs, their solution yields the same pressure behavior that would be obtained from a single-layer reservoir, 
since they considered a single-lateral well.

\subsection{2}

\section{Formulations for Single-Phase Flow in Multilateral Wells}

In reservoirs where areal anisotropy is significant (that is, permeabilities in $x$ - and $y$-directions are remarkably different), productivity of a single lateral horizontal well is influenced by the wellbore drilling direction. Since the main permeability direction is usually unknown a priori, this issue may be mitigated by the use of multilateral wells $[28,30,31]$.

Vo and Madden [29] stated that the algorithm developed by Spath et al. [37] for pressure change in commingled reservoirs with vertical wells is also applicable to the multilateral horizontal well problem. For vertical wells, the algorithm consists of adding the Laplace domain solutions for individual layers. Vo and Madden [29] built a similar procedure for multibranched wells, and presented field example applications. Pressure behavior in each branch is determined using the single-lateral Laplace solution presented by Kuchuk et al. [22]. This method can also determine pressure behavior in multilayer systems, provided that each layer is perforated by at least one wellbore ramification.

An analytical solution for multibranched horizontal wells was proposed by Larsen [30]. This solution, which was also reached using Laplace transform, approximates each wellbore ramification by a uniform influx fracture and is applicable in multilayer reservoirs, with or without formation crossflow.

Using Fourier and Laplace transforms, Yildiz [27] reached a solution in Laplace domain for multilateral wells in single-layer reservoirs. Unlike the formulation proposed by Larsen [30], Yildiz [27] represented each lateral not as a fracture, but using the more realistic approach of infinite conductivity instead. Yildiz [27] discussed the effects of total wellbore length, areal anisotropy and uneven skin factor distribution. In a subsequent work, Yildiz [28] analyzed how other factors such as branches configuration affects well productivity.

Pan et al. [31] developed a real space solution for multilateral wells, based on the solution achieved by Daviau et al. [21] and Ozkan et al. [18]. The formulation consists of applying the solution for each individual branch and then using a suitable wellbore condition (uniform influx or infinite conductivity) to couple the equations for all laterals. Their work presented a comparison between several wellbore configurations and discussed the effects of areal anisotropy on well productivity. Although Pan et al. [31] stated that their formulation applies to multilayer reservoirs (and even showed a field case example), no validation was presented for the multilayer case. 


\subsection{3}

\section{Formulations for Injectivity Tests in Horizontal Wells}

Only a few papers regarding injectivity tests in horizontal wells have been published. Numerical studies have assessed the efficiency of water flooding projects, where the reservoir is drilled by injector and producer horizontal wells $[38,39]$. When it comes to analytical models, the existing solutions can only describe pressure behavior in single-layer reservoirs.

Peres and Reynolds [10] developed a formulation that describes the pressure response during the injection period in single-layer reservoirs. They proposed that the mobility differences between the fluids may be condensed into a term that adjusts the expected single-phase flow pressure change. This additional term is computed after the flood front in each direction has been determined. Their results also showed that pressure response from fluid injection through a horizontal wellbore without formation damage might be estimated by the single-phase solution, unlike what happens in vertical wells.

Boughara and Reynolds [17] developed a formulation for injectivity tests that accounts for non-isothermal effects. Their solution considers that the injected water is at a different temperature than the reservoir and the temperature profile may be approximated by a Heaviside function. Temperature front is determined from the thermal balance and the flood front is computed using non-isothermal Buckley-Leverett [40] theory. Solutions for both vertical and horizontal wells in single-layer reservoirs are presented.

The falloff solution in single-layer reservoirs was reached by Peres et al. [11], applying the rate superposition principle to depict flow-rate history after the well is shut in. Both injection and falloff solutions assume uniform flux along the well.

To the best of the author's knowledge, the formulations achieved by Vo and Madden [29], Larsen [30] and Pan et al. [31] are the only solutions for single-phase flow in multilayer systems with horizontal wells, and there is currently no analytical solution available for the pressure behavior in multilayer reservoirs under two-phase flow through multibranched horizontal wells. Hence, the main purpose of this Chapter is to develop new analytical solutions for analyzing injection/falloff tests under two-phase oil-water flow conditions in stratified reservoirs with multilateral horizontal wells. The proposed solutions extend the already known analytical formulations for injection/falloff tests in single-layer formations $[10,11]$ combined with the model proposed by Pan et al. [31] for single-phase flow in multibranched wells, considering a slightly compressible fluid with constant viscosity and compressibility. 


\section{3}

\section{Injectivity Tests with Multilateral Horizontal Wells}

A reservoir with an arbitrary number of layers was considered. Each layer may present distinct properties from one another. It was assumed no crossflow between layers. Therefore, pressure behavior will be influenced by all layers only if the wellbore presents one ramification per layer. A schematic of the model considered in this study is shown in Fig. 2.1. All computations assume that a consistent set of units is used and that the following simplifying hypotheses are valid:

- Reservoir initially in equilibrium;

- Homogeneous, laterally infinite reservoir, with impermeable vertical boundaries;

- Multilayer reservoir without crossflow, that is, the vertical section of the wellbore is the only flow path between layers;

- Apart from the hydrostatic column, pressure is initially the same in all layers;

- Each wellbore branch is parallel to the horizontal plane, located at an arbitrary distance from the formation bottom

- Wellbore injects at a constant flow-rate of $q_{i n j}$ (flow-rate in each individual horizontal branch, on the other hand, may change in time)

- Infinite conductivity: pressure is uniform along each wellbore branch;

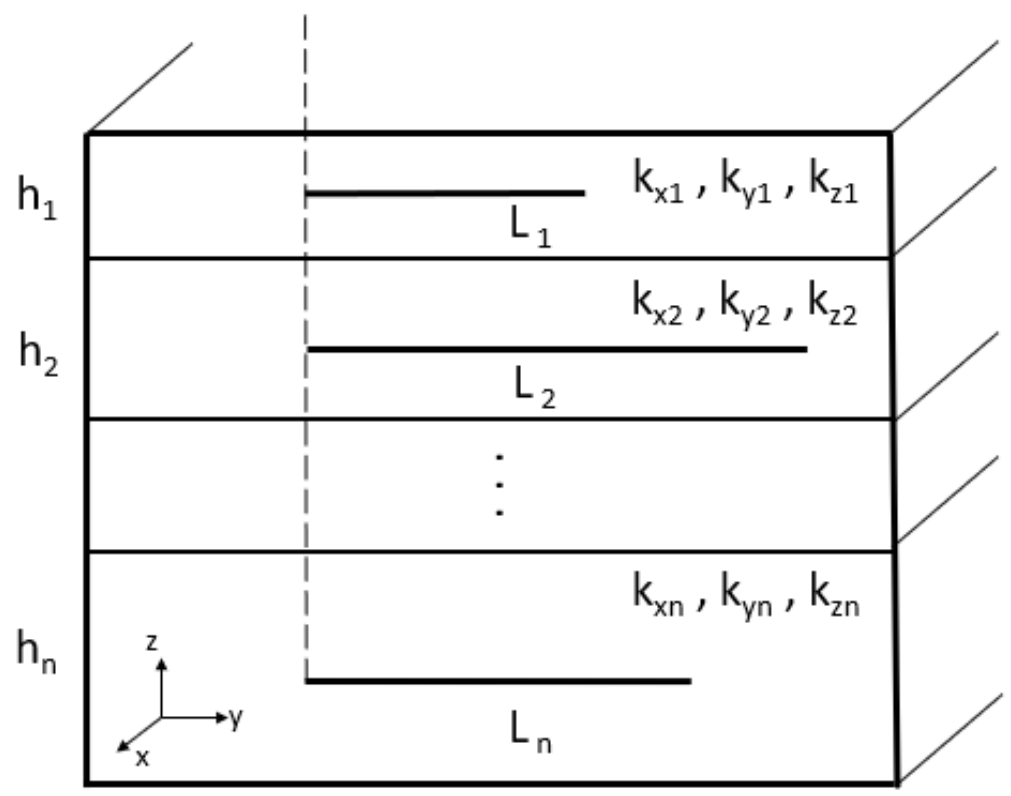

Figure 2.1: Multilayer reservoir model with a multilateral horizontal wellbore 
- Fluids are slightly compressible, having constant viscosity and isothermal compressibility;

- Isothermal flow of oil and water;

- Gravitational, capillary and wellbore storage effects are neglected;

The solutions for two-phase flow through horizontal wells in singlelayer formations were presented by Peres and Reynolds [10] and Peres et al. [11]. In this Section, their solutions will be introduced and, then, extended to multilayer stratified reservoirs. The main insight is to split the bottom hole pressure in two terms: one that encompasses the single-phase oil flow and another that derives from the mobility contrast between oil and water $[12,16,32]$.

\subsection{1}

\section{Injectivity Tests in Single-Layer Reservoirs}

As proposed by Peres and Reynolds [10], pressure change at the wellbore $\left(\Delta p_{w f}\right)$ during injectivity tests in a single-layer reservoir is computed as:

$$
\begin{array}{r}
\Delta p_{w f}(t)=\Delta p_{o}(t)+\frac{q_{\text {inj }}}{\hat{\lambda}_{o} h}\left\{\frac{\pi h}{k_{x} L} \int_{\frac{\pi h}{8}}^{\beta}\left(\frac{\hat{\lambda}_{o}}{\lambda_{t}(x, t)}-1\right) \frac{d x}{h(x)}+\right. \\
\frac{1}{k_{x y}} \int_{L / 2}^{\max \left(\frac{L}{2}, r_{F x y}\right)}\left(\frac{\hat{\lambda}_{o}}{\lambda_{t}(r, t)}-1\right) \frac{d r}{r}+ \\
+\frac{h}{k_{z x} L}\left[\left(\frac{k_{z x}}{k_{\text {skin }}}-1\right) \int_{r_{w}}^{\min \left(r_{s k i n}, r_{F z x}\right)}\left(\frac{\hat{\lambda}_{o}}{\lambda_{t}(r, t)}-1\right) \frac{d r}{r}+\right. \\
\left.\left.\int_{r_{w}}^{\min \left(d z, r_{F z x}\right)}\left(\frac{\hat{\lambda}_{o}}{\lambda_{t}(r, t)}-1\right) \frac{d r}{r}\right]\right\},
\end{array}
$$

where $\hat{\lambda}_{o}$ and $\lambda_{t}$ denote, respectively, the endpoint oil mobility and total mobility (which represent the relative easiness from a given fluid to flow throughout the porous media); $k_{x}, k_{z x}=\sqrt{k_{z} k_{x}}$ and $k_{x y}=\sqrt{k_{x} k_{y}}$ are, respectively, the permeabilities in the $x$-direction, in the $z x$ - and $x y$-planes (the permeability indicates the area within the porous media that is available for fluid to flow); $q_{i n j}$ is the injection flow-rate; $h$ is the reservoir thickness; $L$ is the wellbore length; $r_{w}$ is the wellbore radius; $k_{\text {skin }}$ and $r_{\text {skin }}$ are, respectively, the skin zone permeability and radius (the skin zone consists of a region concentric with the wellbore where permeability is changed due to wellbore perforation and completion); $d z$ is the smallest distance between the reservoir 
and a vertical boundary; $r_{F z x}$ and $r_{F x y}$ represent the flood front radii in the $z x$ - and $x y$-planes, respectively.

The $\Delta p_{o}(t)$ term is determined as depicted in Appendix A.1, and it represents the pressure change that would be obtained if the injected fluid presented the same properties as the reservoir underlying oil. The terms between braces in Eq. (2-1) represent the mobility contrast in the $x$-direction, the $x y$-plane, inside the damaged region and in the $z x$-plane, respectively. Therefore, they may be understood as correction terms that are required due to the differences between water and oil. Integration limits in Eq. (2-1) come from the flooding patterns proposed by Deppe [41]. The first integral upper limit is defined as:

$$
\beta=\min \left[\max \left(\frac{\pi h}{8}, x_{F}(t)\right), \frac{\pi L}{8}\right],
$$

where $x_{F}(t)$ indicates the flood front propagation in the $x$-direction at a given time $t$. The flood fronts in each plane are determined as depicted by Buckley and Leverett [40], using the proper equations for each geometry [10]. Hence, the water front radius in the $z x$-plane is given by:

$$
r_{F z x}(t)=\sqrt{r_{w}^{2}+\frac{q_{i n j} t}{\pi \phi L} f_{w}^{\prime}\left(S_{w}\right)},
$$

where $\phi$ is the reservoir porosity (which represents the fraction of porous media that is not filled by rock) and $f_{w}^{\prime}$ is the fractional flow derivative, which is a function of water saturation $S_{w}$.

In the $x$-direction, the flood front is computed as:

$$
x_{F}(t)=\frac{q_{i n j} t}{2 \pi \phi L h} f_{w}^{\prime}\left(S_{w}\right) .
$$

Finally, in the $x y$-plane:

$$
r_{F x y}(t)=\sqrt{\frac{q_{i n j} t}{\pi \phi h} f_{w}^{\prime}\left(S_{w}\right)} .
$$

Flood front propagation in each direction should be determined from Eqs. (2-3) to (2-5) at all times. However, as the flood front develops, the integrals in Eq. (2-1) become less or more significant, since the integral limits change in time $[10,41]$. Therefore, the flood front propagation in the $x$-direction only starts to influence the pressure change after $x_{F}(t)>\pi h / 8$. On the other hand, the second integral term of Eq. (2-1) will be numerically equal to zero while $r_{F x y}(t) \leq L / 2$. One should also notice that no restriction was made regarding the fractional flow derivative. This means that water saturation may vary inside the swept region, and the saturation profile will fundamentally depend on the relative permeability curves. 


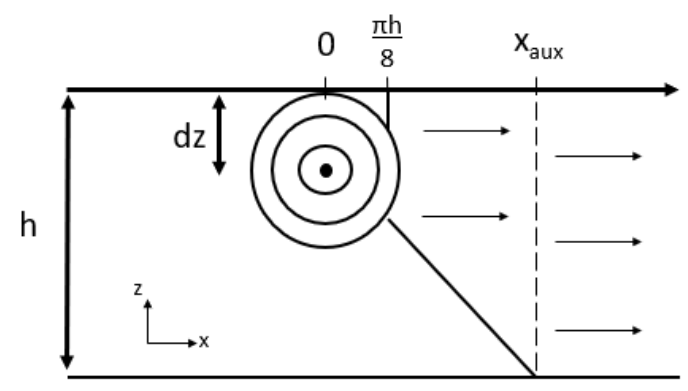

Figure 2.2: Linear flood front propagation from a non centered horizontal well

Eq. (2-1) is derived under the assumption that flow-rate is constant within the flooded region. Peres and Reynolds [10] stated the criteria required for this hypothesis to hold and showed that, in most practical conditions, the flood front is indeed within the steady-state region.

For non-centered wells, the effective flooded thickness varies in the $x$ direction. That is the reason why thickness is expressed as a function of $x$ in the first integral of Eq. (2-1). Fig. 2.2 displays the flood front propagation model that was considered in this work, which was suggested by Peres et al. [11]. For values of $x$ such that $\frac{\pi h}{8} \leq x \leq x_{a u x}$, the effective flooded zone thickness is estimated by a linear interpolation between the reservoir thickness and the smallest distance between the well and a vertical boundary $d z$. The coordinate $x_{\text {aux }}$ is defined as [11]:

$$
x_{\text {aux }}=\frac{\frac{\pi^{2}}{8\left(\frac{h}{2 d z}-1\right)} \ln \left(\frac{h}{2 d z}\right)+\ln \left(\frac{h}{2 \pi d z \sin (\pi d z / h)}\right)}{\frac{\pi}{h}\left[\frac{\frac{h}{2 d z}}{\frac{h}{2 d z}-1} \ln \left(\frac{h}{2 d z}\right)-1\right]} .
$$

\subsection{2}

\section{Injectivity Tests in Multilayer Reservoirs}

The first contribution of this Chapter is to extend the analytical model presented in Section 2.3.1 for commingled reservoirs. The multilayer formulation begins by applying Eq. (2-1) at a given layer $j$ : 


$$
\begin{array}{r}
\Delta p_{w f j}(t)=\Delta p_{o j}(t)+\frac{q_{j}}{\hat{\lambda}_{o_{j}} h_{j}}\left\{\frac{\pi h_{j}}{k_{x j} L_{j}} \int_{\frac{\pi h_{j}}{8}}^{\beta_{j}}\left(\frac{\hat{\lambda}_{o_{j}}}{\lambda_{t_{j}}}-1\right) \frac{d x}{h_{j}(x)}+\right. \\
\frac{1}{k_{x y j}} \int_{L_{j} / 2}^{\max \left(\frac{L_{j}}{2}, r_{F x y_{j}}\right)}\left(\frac{\hat{\lambda}_{o_{j}}}{\lambda_{t_{j}}}-1\right) \frac{d r}{r}+ \\
+\frac{h_{j}}{k_{z x j} L_{j}}\left[\left(\frac{k_{z x j}}{k_{s k i n j}}-1\right) \int_{r_{w}}^{\min \left(r_{s k i n j}, r_{F z x_{j}}\right)}\left(\frac{\hat{\lambda}_{o_{j}}}{\lambda_{t_{j}}}-1\right) \frac{d r}{r}+\right. \\
\left.\left.\int_{r_{w}}^{\min \left(d z, r_{F z x_{j}}\right)}\left(\frac{\hat{\lambda}_{o_{j}}}{\lambda_{t_{j}}}-1\right) \frac{d r}{r}\right]\right\} .
\end{array}
$$

In Eq. (2-7), the $\Delta p_{o j}(t)$ term represents the pressure change due to single-phase oil flow. Considering a stratified reservoir is critical to the development of the proposed formulation. If there were formation crossflow, once the flood front at a given layer had reached a vertical boundary, it would possibly invade adjacent layers. Flood front propagation, then, would be much more complicate to foresee. Since formation crossflow was not accounted for, flood front in each layer may be computed independently and analogously to the single-layer model.

It is important to notice, though, that flow-rate in each wellbore lateral (and, hence, in each layer) may change in time [27, 31]. Thus, the flow-rateelapsed time product in Eqs. (2-3) to (2-5) should be replaced by the time integral of flow-rate. Hence, the water front in the $z x$-plane in layer $j$ is computed as:

$$
r_{F z x_{j}}(t)=\sqrt{r_{w}^{2}+\frac{\int_{0}^{t} q_{j}(\tau) d \tau}{\pi \phi L_{j}} f_{w j}^{\prime}\left(S_{w}\right) .}
$$

The flood front in the $x$-direction in layer $j$ is determined as:

$$
x_{F_{j}}(t)=\frac{\int_{0}^{t} q_{j}(\tau) d \tau}{2 \pi \phi L_{j} h_{j}} f_{w j}^{\prime}\left(S_{w}\right) .
$$

In the $x y$-plane, flood front is evaluated as :

$$
r_{F x y_{j}}(t)=\sqrt{\frac{\int_{0}^{t} q_{j}(\tau) d \tau}{\pi \phi h_{j}} f_{w j}^{\prime}\left(S_{w}\right) .}
$$

Eqs (2-8) to (2-10) evidence that the water front propagation depends 
on the fractional flow derivative. Thus, the flood front shape is intrinsically related to the fractional flow derivative curve which, in its turn, is obtained from the relative permeability data. Thereby, water saturation inside the swept region is not constant, and depends on the relative permeability curves.

Inspired by the analytical solution for vertical wells presented by Barreto et al. [16], a weighting variable $A_{j}(t)$ is now introduced:

$$
\begin{array}{r}
A_{j}(t)=\frac{1}{\hat{\lambda}_{o_{j}} h_{j}}\left\{\frac{\pi h_{j}}{k_{x j} L_{j}} \int_{\frac{\pi h_{j}}{8}}^{\beta_{j}}\left(\frac{\hat{\lambda}_{o_{j}}}{\lambda_{t_{j}}(x, t)}-1\right) \frac{d x}{h_{j}(x)}+\right. \\
\frac{1}{k_{x y j}} \int_{L_{j} / 2}^{\max \left(\frac{L_{j}}{2}, r_{F x y_{j}}\right)}\left(\frac{\hat{\lambda}_{o_{j}}}{\lambda_{t_{j}}(r, t)}-1\right) \frac{d r}{r}+ \\
+\frac{h_{j}}{k_{z x j} L_{j}}\left[\left(\frac{k_{z x j}}{k_{s k i n j}}-1\right) \int_{r_{w}}^{\min \left(r_{s k i n j}, r_{F z x_{j}}\right)}\left(\frac{\hat{\lambda}_{o_{j}}}{\lambda_{t_{j}}(r, t)}-1\right) \frac{d r}{r}+\right. \\
\left.\left.\int_{r_{w}}^{\min \left(d z, r_{F z x_{j}}\right)}\left(\frac{\hat{\lambda}_{o_{j}}}{\lambda_{t_{j}}(r, t)}-1\right) \frac{d r}{r}\right]\right\} .
\end{array}
$$

The weighting variable $A_{j}(t)$ groups the three integral terms that represent the flood front propagation in Eq. (2-7) into a single coefficient, simplifying the notation on subsequent computations. Replacing Eq. (2-11) in Eq. (2-7):

$$
\Delta p_{w f j}(t)=\Delta p_{o j}(t)+q_{j} A_{j}(t)
$$

As demonstrated in Appendix A.2, for an infinite conductivity wellbore, the $\Delta p_{o j}(t)$ term associated with the single-phase oil displacement is, in fact, the same for all layers [31]:

$$
\Delta p_{o_{1}}(t)=\Delta p_{o_{2}}(t)=\cdots=\Delta p_{o_{n}}(t)=\Delta p_{o}(t), \quad \forall j=1, \ldots, n .
$$

For this reason, it will be hereafter denoted simply as $\Delta p_{o}(t)$. Besides, the considered model states that pressure change is also equal in all layers, except for the hydrostatic column:

$$
\Delta p_{w f_{1}}(t)=\Delta p_{w f_{2}}(t)=\cdots=\Delta p_{w f_{n}}(t)=\Delta p_{w f}(t), \quad \forall j=1, \ldots, n .
$$

Thus, rearranging Eq. (2-12), layer flow-rate may be computed as: 


$$
q_{j}=\frac{\Delta p_{w f}(t)-\Delta p_{o}(t)}{A_{j}(t)}
$$

Summing up all layer flow-rates:

$$
q_{i n j}=\sum_{j=1}^{n} q_{j}=\left(\Delta p_{w f}(t)-\Delta p_{o}(t)\right) \sum_{j=1}^{n} \frac{1}{A_{j}(t)} .
$$

Rearranging Eq. (2-16):

$$
\Delta p_{w f}(t)=\Delta p_{o}(t)+\frac{q_{i n j}}{\sum_{j=1}^{n} A_{j}^{-1}(t)} .
$$

Eq. (2-17) depicts the pressure behavior in multilayer systems under water injection. In single-layer reservoirs, it yields the same result as equation $(2-1)$, which assures that the suggested formulation is applicable to reservoirs with any number of layers. Furthermore, Eq. (2-17) is analogous to the formulation for injectivity tests in multilayer reservoirs with vertical wellbores [16]. The definition of $A_{j}(t)$ and the computation of $\Delta p_{o}(t)$, however, differ from the corresponding ones proposed by Barreto et al. [16], since a multilateral horizontal well is considered in this work.

A computational implementation of the solution derived in this Section may be obtained from the following algorithm:

- For each time step:

1. Build the linear system given by Eq. (A-8),

2. Determine layer flow-rates at the current time step by solving the linear system

3. Compute $\Delta p_{o}(t)$ using Eq. (A-7)

4. For each layer, compute the flood front in each direction using the theory from Buckley and Leverett [40]

5. For all layers, compute the $A_{j}(t)$ coefficients as defined in Eq. (2-11)

6. Compute $\Delta p_{w f}(t)$ using Eq. (2-17)

- Repeat steps 1 to 6 for the next time step 


\subsection{3}

\section{Formulation for Falloff Tests}

Peres et al. [11] developed the solution for falloff tests in single-layer reservoirs with horizontal wells. They demonstrated that the flow-rate profile along the flooded zone directly influences pressure change during falloff (denoted as $\left.\Delta p_{w s}\right)$. Considering that the well is shut in at $t=t_{p}$, the pressure change after a certain shut-in time $\Delta t=t-t_{p}$ may be computed as:

$$
\begin{array}{r}
\Delta p_{w s}(\Delta t)=\Delta p_{o s}(\Delta t)+\frac{1}{h}\left\{\frac{\pi h}{k_{x} L} \int_{\frac{\pi h}{8}}^{\beta}\left(\frac{q_{s}}{\lambda_{t}}-\frac{\hat{q}_{o s}}{\hat{\lambda}_{o}}\right) \frac{d x}{h(x)}+\right. \\
\frac{1}{k_{x y}} \int_{L / 2}^{\max \left(\frac{L}{2}, r_{F x y}\right)}\left(\frac{q_{s}}{\lambda_{t}}-\frac{\hat{q}_{o s}}{\hat{\lambda}_{o}}\right) \frac{d r}{r}+ \\
\frac{h}{k_{z x} L}\left[\left(\frac{k_{z x}}{k_{s k i n}}-1\right) \int_{r_{w}}^{\min \left(r_{s k i n}, r_{F z x}\right)}\left(\frac{q_{s}}{\lambda_{t}}-\frac{\hat{q}_{o s}}{\hat{\lambda}_{o}}\right) \frac{d r}{r}+\right. \\
\left.\left.\int_{r_{w}}^{\min \left(d z, r_{F z x}\right)}\left(\frac{q_{s}}{\lambda_{t}}-\frac{\hat{q}_{o s}}{\hat{\lambda}_{o}}\right) \frac{d r}{r}\right]\right\} .
\end{array}
$$

In Eq. (2-18), the subscript $s$ denotes the falloff period, whereas $\hat{q}_{o s}$ and $q_{s}$ denote, respectively, oil flow-rate and total flow-rate, which is the sum of oil and water flow-rates. Again, the $\Delta p_{o s}(\Delta t)$ term refers to the single-phase contribution and should be evaluated as detailed in Appendix A.1.

After the well is shut in, a zero-rate pulse propagates throughout the reservoir [12]. Therefore, flow-rate inside the flooded region becomes a function of position and shut-in time [11]. Thus, it must be written under the integral sign. In Eq. (2-18), the flood fronts are assumed to remain constant [42, 43]. Hence, the integral limits in Eq. (2-18) also remain constant. Therefore, the first and second integral terms of Eq. (2-18) may be numerically equal to zero if the well is shut-in before the water front reaches a vertical boundary; that is, if shut-in time $t_{p}$ is short enough so that the water front propagation is still at the "first radial" regime at the moment of shut-in [17].

During falloff, oil flow-rate $\left(\hat{q}_{o s}\right)$ considering radial flow may be estimated from the logarithmic approximation of the line-source solution [11]:

$$
\hat{q}_{o s}(r, \Delta t)=q_{i n j}\left[\exp \left(-\frac{\phi c_{t} r^{2}}{4 k \hat{\lambda}_{o}\left(t_{p}+\Delta t\right)}\right)-\exp \left(-\frac{\phi c_{t} r^{2}}{4 k \hat{\lambda}_{o}(\Delta t)}\right)\right]
$$


where $c_{t}$ stands for total compressibility. For linear flow, oil flow-rate may be determined as [11]:

$$
\hat{q}_{o s}(x, \Delta t)=q_{i n j}\left[\operatorname{erfc}\left(\frac{x}{2} \sqrt{\frac{\phi c_{t}}{4 k \hat{\lambda}_{o}\left(t_{p}+\Delta t\right)}}\right)-\operatorname{erfc}\left(\frac{x}{2} \sqrt{\frac{\phi c_{t}}{4 k \hat{\lambda}_{o}(\Delta t)}}\right)\right]
$$

Total flow-rate $\left(q_{s}\right)$ is computed by replacing the endpoint oil mobility by total mobility at the considered position in Eqs. (2-19) and (2-20).

Another goal of this Chapter is to extend the falloff solution given by Eq. (2-18) to commingled reservoirs. For multilayer formations, the application of Eq. (2-18) at a given layer $j$ yields:

$$
\begin{array}{r}
\Delta p_{w s j}(\Delta t)=\Delta p_{o s j}(\Delta t)+\frac{1}{h_{j}}\left\{\frac{\pi h_{j}}{k_{x j} L_{j}} \int_{\frac{\pi h_{j}}{8}}^{\beta_{j}}\left(\frac{q_{s j}}{\lambda_{t}}-\frac{\hat{q}_{o s j}}{\hat{\lambda}_{o}}\right) \frac{d x}{h_{j}(x)}+\right. \\
\frac{1}{k_{x y j}} \int_{L_{j} / 2}^{\max \left(\frac{L_{j}}{2}, r_{F x y j}\right)}\left(\frac{q_{s j}}{\lambda_{t}}-\frac{\hat{q}_{o s j}}{\hat{\lambda}_{o}}\right) \frac{d r}{r}+ \\
\frac{h_{j}}{k_{z x j} L_{j}}\left[\left(\frac{k_{z x j}}{k_{s k i n j}}-1\right)\right. \\
\int_{r_{w}}^{\min \left(r_{s k i n j}, r_{F z x j}\right)}\left(\frac{q_{s j}}{\lambda_{t}}-\frac{\hat{q}_{o s j}}{\hat{\lambda}_{o}}\right) \frac{d r}{r}+ \\
\min \left(d z_{j}, r_{F z x j}\right) \\
\left.\left.\left.\int_{r_{t}}-\frac{q_{s j}}{\hat{q}_{o s j}}\right) \frac{d r}{r}\right]\right\} .
\end{array}
$$

To obtain the formulation for pressure behavior, it will be assumed that the zero-rate pulse equally propagates in all layers, even though layer properties might be different. The same strong assumption was made by Bela et al. [12] to develop the solution for falloff tests in stratified reservoirs with vertical wells. Although this hypothesis is essential to the development of the falloff solution, it might not be realistic at early times in reservoirs where layer properties are remarkably different $[5,44]$. The consequence of this assumption on the solution will be discussed in Section 2.4. Thereby, under this hypothesis, total and oil flow-rates in layer $j$ may be respectively expressed in terms of layer flow-rate fraction $\left(q_{D p j}\right)$ as:

$$
q_{s j}(r, \Delta t)=q_{D p j} q_{s}(r, \Delta t)
$$

and

$$
\hat{q}_{o s j}(r, \Delta t)=q_{D p j} \hat{q}_{o s}(r, \Delta t),
$$

where $q_{s}$ and $\hat{q}_{o s}$ stand for the sum total and oil flow-rates in all layers. 
Those flow-rates are estimated from Eqs. (2-19) and (2-20), using the reservoir equivalent properties [12].

Moreover, introducing a weighting variable $R_{j}(\Delta t)$ defined as:

$$
\begin{gathered}
R_{j}(\Delta t)=\frac{1}{h_{j}}\left\{\frac{\pi h_{j}}{k_{x j} L_{j}} \int_{\frac{\pi h_{j}}{8}}^{\beta_{j}}\left(\frac{q_{s}}{\lambda_{t}}-\frac{\hat{q}_{o s}}{\hat{\lambda}_{o}}\right) \frac{d x}{h_{j}(x)}+\right. \\
\frac{1}{k_{x y j}} \int_{L_{j} / 2}^{\max \left(\frac{L_{j}}{2}, r_{F x y j}\right)}\left(\frac{q_{s}}{\lambda_{t}}-\frac{\hat{q}_{o s}}{\hat{\lambda}_{o}}\right) \frac{d r}{r}+ \\
\frac{h_{j}}{k_{z x j} L_{j}}\left[\left(\frac{k_{z x j}}{k_{s k i n j}}-1\right) \int_{r_{w}}^{\min \left(r_{s k i n j}, r_{F z x j}\right)}\left(\frac{q_{s}}{\lambda_{t}}-\frac{\hat{q}_{o s}}{\hat{\lambda}_{o}}\right) \frac{d r}{r}+\right. \\
\left.\left.\int_{r_{w}}^{\min \left(d z_{j}, r_{F z x j}\right)}\left(\frac{q_{s}}{\lambda_{t}}-\frac{\hat{q}_{o s}}{\hat{\lambda}_{o}}\right) \frac{d r}{r}\right]\right\} .
\end{gathered}
$$

Analogously to injection period formulation, the weighting variable $R_{j}(\Delta t)$ defined in Eq. (2-24) aims to encompass the three integral terms of Eq. (2-21) into a single coefficient to simplify the notation. Then, layer flowrate fraction may be written out of the integral sign and Eq. (2-21) may be expressed as:

$$
\Delta p_{w s j}(\Delta t)=\Delta p_{o s j}(\Delta t)+q_{D p j} R_{j}(\Delta t) .
$$

The hypothesis that the zero-rate pulse equally propagates in all layers was required to derive Eq. (2-25) using the $R_{j}(\Delta t)$ coefficient defined in Eq. (2-24) and layer flow-rate fraction $q_{D p j}$. Pressure change related to the singlephase oil contribution (that is, the $\Delta p_{o s j}(\Delta t)$ term), however, was not affected by this assumption. Therefore, Eq. (2-25) requires that layer flow-rate fractions remain constant inside the flooded region only.

Once again, wellbore pressure change in all layers is assumed to be the same by model hypothesis. Moreover, the term associated with single-phase oil flow $\left(\Delta p_{o s j}(\Delta t)\right)$ is also the same in all layers. Thereby, flow-rate fraction in layer $j$ may be computed as:

$$
q_{D p j}=\frac{\Delta p_{w s}(\Delta t)-\Delta p_{o s}(\Delta t)}{R_{j}(\Delta t)} .
$$

By definition, the sum of all layer flow-rate fractions must be equal to one. Thus: 


$$
1=\sum_{j=1}^{n} q_{D p j}=\left(\Delta p_{w s}(\Delta t)-\Delta p_{o s}(\Delta t)\right) \sum_{j=1}^{n} \frac{1}{R_{j}(\Delta t)} .
$$

Rearranging Eq. (2-27):

$$
\Delta p_{w s}(\Delta t)=\Delta p_{o s}(\Delta t)+\frac{1}{\sum_{j=1}^{n} R_{j}^{-1}(\Delta t)} .
$$

Thus, wellbore pressure during falloff tests in multilayer reservoirs with multilateral horizontal wells may be evaluated from Eq. (2-28). Similar to the formulation for the injection period, this solution is analogous to the model for vertical wells [12], provided that $\Delta p_{o s}(\Delta t)$ is determined using the formulation developed by Pan et al. [31] for multilateral horizontal wells and the $R_{j}(\Delta t)$ coefficients are computed according to Eq. (2-24).

The computational implementation of the solution presented in this Section should proceed according to the following algorithm:

- For each layer, store the flood front profile at the moment of shut-in $\left(t=t_{p}\right)$

- For each time step:

1. Compute $\Delta p_{o s}(\Delta t)$ using Eq. (A-7) and the superposition principle

2. For all layers, compute the $R_{j}(\Delta t)$ coefficients as defined in Eq. $(2-24)$

3. Compute $\Delta p_{w s}(\Delta t)$ using Eq. (2-28)

- Repeat steps 1 to 3 for the next time step

\subsection{4}

\section{Pressure Derivative Behavior}

This Section presents theoretical pressure derivative responses for different flow regimes that may be observed during injectivity/falloff tests in multilayer reservoirs with multilateral horizontal wells. Peres and Reynolds [10] showed that two-phase flow in reservoirs with horizontal wells might present several distinct flow regimes, which depend not only on the pressure transient zone but also on the flooded region.

They denoted the first regime as "first radial - first radial", since both the pressure transient pulse and the flood front are propagating in the vertical plane perpendicular to the wellbore. During this flow regime, pressure derivative reflects oil properties, and behaves similar to the early radial regime observed at a single-phase flow. Therefore, pressure derivative during this flow 
regime attains a constant level that is related to the equivalent properties in the vertical plane:

$$
\frac{\partial \Delta p_{w f}}{\partial \ln (t)} \approx \frac{1}{2} \frac{q_{i n j}}{L_{t}\left(k_{z x}\right)_{e q} \hat{\lambda}_{o}},
$$

where $L_{t}$ stands for the wellbore total effective length and $\left(k_{z x}\right)_{e q}$ is the equivalent permeability in the $z x$-plane, which is defined as:

$$
\left(k_{z x}\right)_{e q}=\frac{\sum_{j=1}^{n} k_{z x j} L_{j}}{L_{t}} .
$$

As the injection goes on, the flood front propagates. In reservoirs with formation damage, eventually the flood front overcomes the skin zone radius, yielding a characteristic shift in pressure derivative [32]. During this blunt shift, pressure derivative may even attain negative values [10].

After the early radial flow, a linear regime develops in each layer. However, the linear flow might start and end at different times in each layer. Therefore, it is not possible to state a priori that the characteristic derivative signature related to this flow regime will be identified.

The linear regime is followed by a late radial flow. Since the reservoir is assumed to be laterally infinite, eventually pressure diffusion will simultaneously propagate through the horizontal plane in all layers. Pressure derivative during this late radial flow period will depend on the flood front radius in each layer. Using the same notation from Peres and Reynolds [10], either a "second radial - first radial" or a "second radial - first linear" or a "second radial - second radial" flow regime may be observed. Assuming that the "second radial - first radial" flow develops in all layers, pressure derivative is approximated by:

$$
\frac{\partial \Delta p_{w f}}{\partial \ln (t)} \approx \frac{1}{2} \frac{q_{i n j}}{h_{t}\left(k_{x y}\right)_{e q} \hat{\lambda}_{o}}\left(1+\frac{h_{t}}{L_{t}} \frac{(1-\hat{M})}{\hat{M}}\right),
$$

where $h_{t}$ stands for the reservoir total thickness and $\left(k_{z x}\right)_{e q}$ is the equivalent permeability in the $x y$-plane, which is defined as:

$$
\left(k_{x y}\right)_{e q}=\frac{\sum_{j=1}^{n} k_{x y j} h_{j}}{h_{t}} .
$$

where $\hat{M}$ stands for the endpoint mobility ratio, defined as $\hat{M}=\hat{\lambda}_{w} / \hat{\lambda}_{o}$, and $\hat{\lambda}_{w}$ represents the endpoint water mobility. The expression for pressure derivative while the "second radial - second radial" regime occurs in all layers is given by:

$$
\frac{\partial \Delta p_{w f}}{\partial \ln (t)} \approx \frac{1}{2} \frac{q_{i n j}}{h_{t}\left(k_{x y}\right)_{e q} \hat{\lambda}_{o}}\left(1+\frac{h_{t}}{2 L_{t} \hat{M}} \frac{(1-\hat{M})}{\hat{M}}\right) .
$$

Approximations for the other flow regimes may be obtained by adapting the generalized expression for pressure derivative in single-layer reservoirs 
achieved by Peres and Reynolds [10].

Pressure derivative during falloff should be taken with respect to the equivalent time $t_{e}=\frac{t_{p} \Delta t}{t_{p}+\Delta t}[11,12,17]$. During early falloff times, pressure change is mostly influenced by properties in the vertical plane perpendicular to the wellbore. At very early times, one might expect that pressure derivative attains a constant level associated with endpoint water properties. Thus:

$$
\frac{\partial \Delta p_{w s}}{\partial \ln \left(t_{e}\right)} \approx \frac{1}{2} \frac{q_{i n j}}{L_{t}\left(k_{z x}\right)_{e q} \hat{\lambda}_{w}} .
$$

Based on the formulation for buildup analysis from conventional well testing [6], Eq. (2-34) is expected to be valid only if the wellbore is shutin while the early radial flow is still occurring. Moreover, as stated by Peres et al. [11], early-time pressure derivative reflects the total mobility inside the damaged region. Therefore, pressure derivative level does not necessarily match neither the single-phase oil level nor the single-phase water level. Besides, it is important to notice that assuming the zero transient rate diffusion equally propagates in all layers may lead to errors at early falloff times [12].

It is important to notice that, since flood front is assumed to remain stationary during falloff, skin effects only influence pressure change at the wellbore while flow-rate inside the damaged zone is non-zero. Therefore, the damaged zone presents a very limited effect on falloff pressure derivative. As a result, the characteristic blunt derivative shift that occurs during injection is not observed during falloff.

As flow throughout the reservoir ends, pressure derivative gradually becomes closer to the single-phase oil solution $[11,12,17]$. Thus, at late falloff times, pressure behavior is given by:

$$
\frac{\partial \Delta p_{w s}}{\partial \ln \left(t_{e}\right)} \approx \frac{1}{2} \frac{q_{i n j}}{h_{t}\left(k_{x y}\right)_{e q} \hat{\lambda}_{o}} .
$$

Eq. (2-35) represents the same level obtained from the single-phase oil solution $[4,18]$.

\subsection{5}

\section{Discussion on the Main Assumptions}

The developed solutions for injectivity and falloff tests in multilayer reservoirs were achieved considering some simplifying hypotheses, as enumerated before. This Section briefly comments the most relevant assumptions and how they affect the proposed model.

The reservoir was assumed to be composed of stratified layers. Despite the fact that formation crossflow may be relevant in some reservoirs, at short times the vertical flow between layers is negligible [5, 44]. Furthermore, accounting 
for crossflow effects increases the problem complexity. For this reason, the single-phase models proposed by Vo and Madden [29] and Pan et al. [31] also assume stratified reservoirs. Flood front computations are particularly more complicated in reservoirs with crossflow, as water may flow from one layer to another. Therefore, flood front in each layer would depend on adjacent layers as well, and Eqs. (2-8) to (2-10) would be applicable only at early-times. Handling the vertical water flow between layers is out of the scope of this work.

Including capillary effects would change the flood front as well, yielding a smoother profile in regions further from the wellbore. However, mobility profile in regions closer to the wellbore present a more meaningful influence on pressure change, due to the $1 / r$ and $1 / h(x)$ terms that appear inside the integrals defined in Eqs. (2-11) and (2-24). Thus, the capillary forces are not expected to play a significant role in pressure change at the wellbore [11].

Gravitational forces were also neglected. Although a more rigorous solution could be obtained by accounting for the gravitational effect, if the reservoir thickness is not very large, the influence of gravity in pressure response is significantly smaller than the pressure diffusion due to water injection [25]. For this reason, analytical models for horizontal wells typically neglect gravitational forces $[4,6,10,11,18,21,24,36]$.

As for the wellbore hydraulics, the solutions presented in this Chapter assume infinite conductivity, which considers that pressure is uniform along the horizontal branches, but fluid influx varies. This assumption is more realistic than the uniform influx model, which accounts for a uniform fluid influx along the well, while pressure distribution changes. However, a more accurate description of wellbore hydraulics is obtained using a finite conductivity model $[22,33]$, which assumes that both pressure and fluid inflow are not uniform.

Wang and Zhan [26] discussed the effects of frictional and kinetic losses inside the wellbore. Chen et al. [25] showed that wellbore hydraulics may also be relevant in fractured systems. Nonetheless, this Chapter is focused on extending the solution for injectivity/falloff tests to multilayer systems and discuss the main effects of water injection through horizontal wells. In this sense, the infinite conductivity wellbore model consists of a simple but yet adequate hypothesis, considering the objectives of this work. One should also notice that the $A_{j}(t)$ and $R_{j}(\Delta t)$ as defined in Eqs. (2-11) and (2-24), respectively, are related to the mobility differences inside the flooded zone, rather than inside the wellbore. This means that they are not directly affected by wellbore hydraulic effects. Hence, an improved model that accounts for wellbore hydraulics effects may be achieved by using the analytical solutions from Chen et al. [25] or Wang and Zhan [26] to evaluate $\Delta p_{o}(t)$ and $\Delta p_{o s}(\Delta t)$ 
in Eqs. (2-17) and (2-28), respectively.

Finally, neglecting wellbore storage is also a significantly strong assumption. This assumption becomes particularly less accurate in cases where the pressure gauge is placed far from the formation top. Still, many analytical formulations for reservoirs with horizontal wells also neglect wellbore storage effects for simplicity $[6,18,33,36]$. Furthermore, this Chapter also aims to evaluate some early time features that could be masked by wellbore storage effects. For instance, the blunt derivative shift that occurs during injection in reservoirs with formation damage, and the validity of the early-time derivative approximations given by Eqs. (2-29) and (2-34). Previous models for two-phase flow usually assume no wellbore storage so that the early time differences with respect to single-phase flow can be properly analyzed $[9,10,11,12,15,16]$. Therefore, neglecting wellbore storage, albeit unrealistic, allows the identification of pressure derivative features that could otherwise be masked.

\section{4}

\section{Model Validation}

The accuracy of the solutions detailed in Section 2.3 was assessed by comparison to a finite difference-based commercial flow simulator [45]. The employed simulation grid consists of 101 x 101 blocks in the horizontal plane. In the vertical direction, each layer is represented by 11 grid blocks. Blocks containing the wellbore have dimensions $0.6 \mathrm{~m}$ in the $x$-direction, $5 \mathrm{~m}$ in the $y$-direction (which is parallel to the wellbore axis) and $0.6 \mathrm{~m}$ in the $z$-direction. Blocks are progressively coarser as the distance from the wellbore increases. Reservoir grid was 7,000 m wide ( $x$-direction) and 2,000 m long ( $y$-direction). A hybrid grid refinement of 3 (radial direction) by 1 (angular direction) by 4 (well axis direction) was employed at all grid blocks containing the wellbore. A Cartesian refinement of 2 by 2 by 2 was also applied at their neighbors.

A $0.0762 \mathrm{~m}$ wellbore radius was employed. Values for oil and rock compressibilities were $1.14 \cdot 10^{-4}\left(\mathrm{kgf} / \mathrm{cm}^{2}\right)^{-1}$ and $8.0 \cdot 10^{-5}\left(\mathrm{kgf} / \mathrm{cm}^{2}\right)^{-1}$, respectively. It was considered that water viscosity is equal to $0.52 \mathrm{cP}$ and a water compressibility of $4.04 \cdot 10^{-5}\left(\mathrm{kgf} / \mathrm{cm}^{2}\right)^{-1}$. Other reservoir properties may be observed in Table 2.1. Fig. 2.3 shows the relative permeability curves, which are assumed to be the same in all layers. It is important to highlight that it was not required at any step of Section 2.3 that relative permeability data should be the same in all layers. Nonetheless, in a field application it might be challenging to individually determine relative permeability curves for each layer.

In Cases $\mathrm{A}$ and $\mathrm{B}$, it was assumed that water is injected during a $96 \mathrm{~h}$ period, followed by a 96 h-long shut-in period. Case C, in its turn, required a 


\begin{tabular}{|ccccccccccc|}
\hline Case & $\begin{array}{c}q_{i n j} \\
\left(\mathrm{~m}^{3} / \mathrm{d}\right)\end{array}$ & $\begin{array}{c}\mu_{o} \\
(\mathrm{cP})\end{array}$ & $\phi_{j}$ & $\begin{array}{c}k_{x y j} \\
(\mathrm{mD})\end{array}$ & $\begin{array}{c}k_{z j} \\
(\mathrm{mD})\end{array}$ & $\begin{array}{c}k_{s j} \\
(\mathrm{mD})\end{array}$ & $S_{j}$ & $\begin{array}{c}L_{j} \\
(\mathrm{~m})\end{array}$ & $\begin{array}{c}d z_{j} \\
(\mathrm{~m})\end{array}$ & $\begin{array}{c}h_{j} \\
(\mathrm{~m})\end{array}$ \\
\hline \multirow{2}{*}{$\mathrm{A}$} & \multirow{2}{*}{1500} & \multirow{2}{*}{5.7} & 0.23 & 500 & 250 & 200 & 1.3 & 100 & 10 & 20 \\
& & & 0.27 & 1450 & 725 & 200 & 7.1 & 120 & 5 & 15 \\
\hline \multirow{2}{*}{$\mathrm{B}$} & \multirow{2}{*}{2000} & \multirow{2}{*}{3.8} & 0.24 & 590 & 320 & 135 & 3.4 & 200 & 10 & 20 \\
& & & 0.19 & 940 & 610 & 135 & 7.0 & 150 & 12.5 & 25 \\
\hline \multirow{2}{*}{$\mathrm{C}$} & \multirow{2}{*}{1500} & \multirow{2}{*}{1.1} & 0.23 & 500 & 250 & 150 & 2.9 & 100 & 10 & 20 \\
& & & 0.27 & 1450 & 725 & 150 & 12.4 & 120 & 5 & 15 \\
\hline
\end{tabular}

Table 2.1: Reservoir parameters

shorter injection period, to avoid the start of the boundary dominated flowregime in the numerical simulator. Thus, the shut in time $t_{p}$ was set as $24 \mathrm{~h}$. Falloff period also lasts $24 \mathrm{~h}$.

It was considered that well drilling and completion change the permeability around it, representing the skin effect. Gridblocks containing the wellbore were built with $0.6 \mathrm{~m}$ width and $0.6 \mathrm{~m}$ height (that is, distance between the wellbore and a gridblock face is $0.3 \mathrm{~m}$ ) for cases A and B. Permeability inside those blocks was changed to represent the damaged region. Skin zone in the analytical solution was modeled defining the skin radius required so that the cross-sectional damaged region would present the same area as the numerical simulator, yielding a skin radius of $0.35 \mathrm{~m}$. For Case C, the gridblock containing the well presents height and width equal to $1.0 \mathrm{~m}$. Therefore, the skin zone radius in the analytical model was defined as $0.56 \mathrm{~m}$.

Figs. 2.4 and 2.5 display the results for pressure and pressure derivative obtained from the proposed formulation considering the reservoir properties of Case A. The dashed lines denote the theoretical constant pressure deriva-

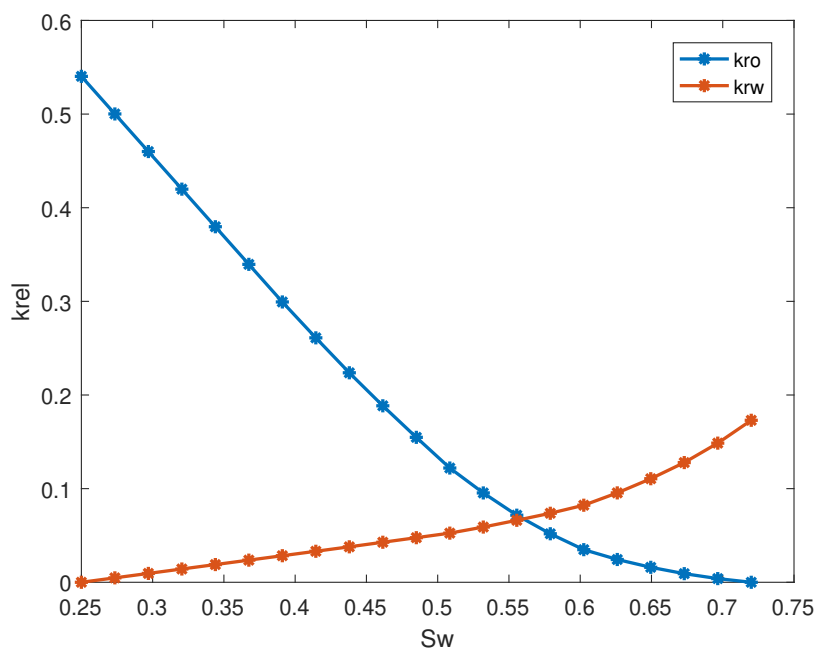

Figure 2.3: Relative permeability curves 


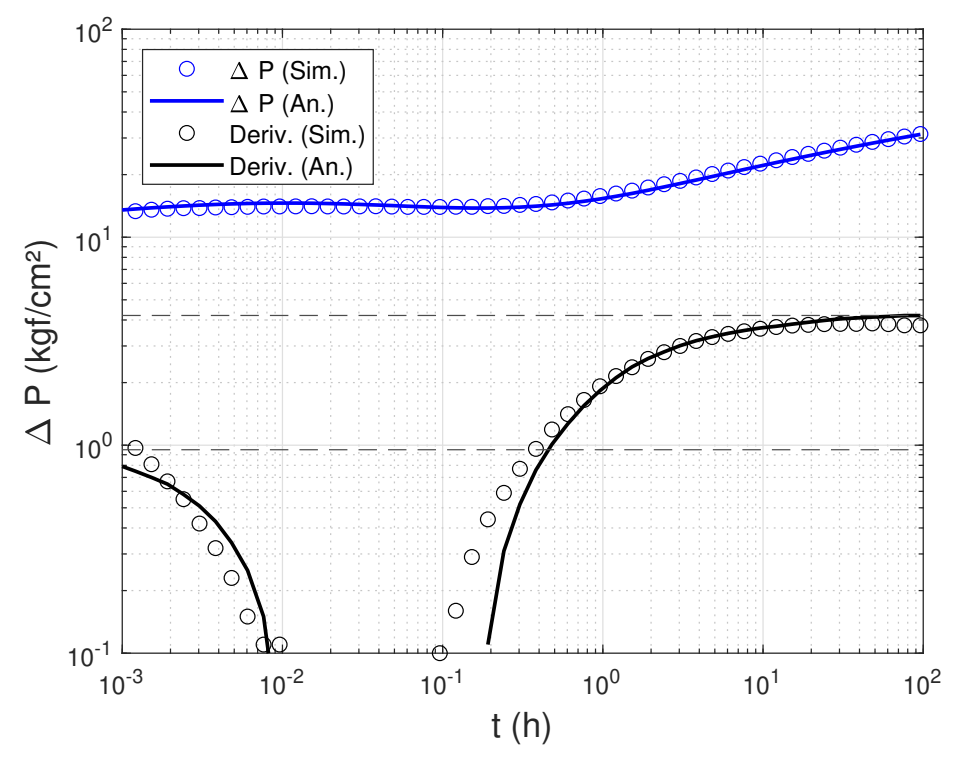

Figure 2.4: Pressure data for Case A during injection

tive levels evaluated as detailed in Section 2.3.4. Early-time derivative was determined using Eqs. (2-29) and (2-34). Late-time derivative level during injection was computed using Eq. (2-31), as the flood front profile indicated that the "second radial - first radial" flow regime was occurring at the moment of shut-in. During falloff, Eq. (2-35) was used to determine the late-time pressure derivative. Pressure derivative during falloff was taken with respect to the equivalent time $t_{e}=\frac{t_{p} \Delta t}{t_{p}+\Delta t}$ and plotted against the elapsed time $\Delta t$.

A good agreement between both analytical and numerical pressure curves is observed, although pressure derivative presents slight divergences. The

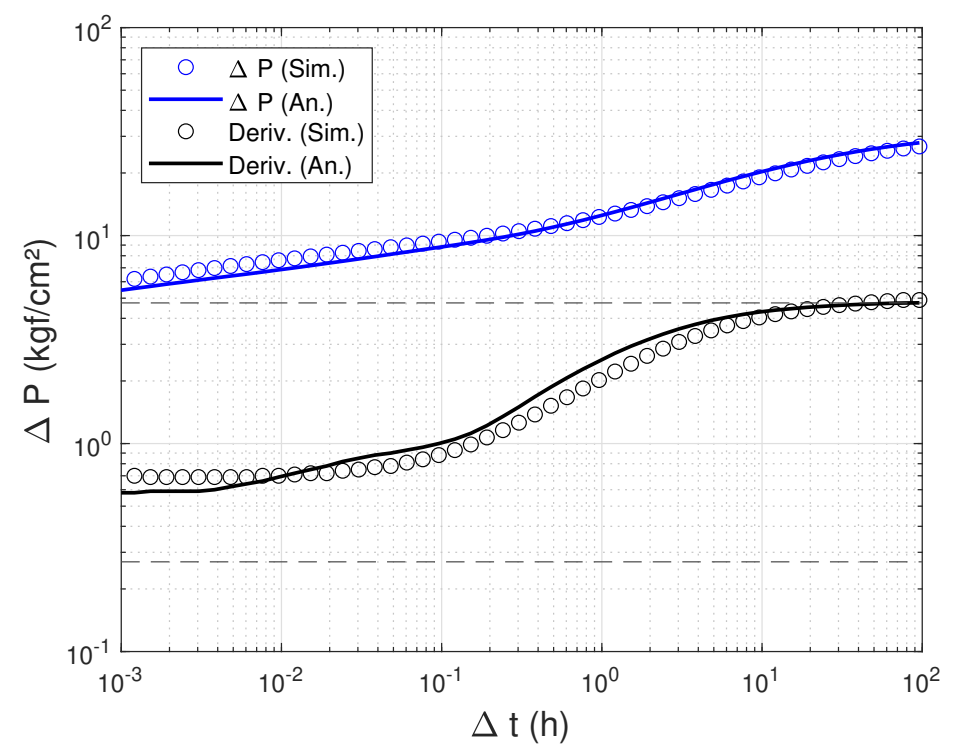

Figure 2.5: Pressure data for Case A during falloff 
main reason for the derivative mismatch is given by the distinct skin zone representation in the flow simulator and in the proposed model. While the former depicts the damaged region as cubes, the latter accounts for a cylindrical skin zone radius. Moreover, the mismatch identified at falloff intermediatetimes may be related to the employed grid refinement. Divergences at early falloff times might also be associated with the hypothesis that the zero-rate pulse equally propagates in all layers. Nonetheless, the overall agreement is quite good.

Pressure derivative profile indicates that the first radial flow regime did not last long enough so that a clear constant derivative level could be noticed at early injection times. During falloff, pressure derivative is approximately constant until $\Delta t \simeq 0.01 \mathrm{~h}$. However, as explained in Section 2.3.4, this level reflects total mobility inside the flooded zone, rather than the properties of oil or water. It is important to recall that the duration of early-time radial flow is directly influenced by the wellbore length and formation thickness $[18,21]$. Thus, in cases where the wellbore length is higher, the early-time pressure derivative level may be observed. Early-time derivative during falloff fails to attain the theoretical level, since a late-radial flow was occurring at the moment of shut-in [6]. On the other hand, at late-times pressure derivative clearly attains the constant levels forecast by Eq. (2-31) during injection and Eq. (2-35) during falloff.

During injection, pressure derivative attains negative values between $t \simeq 0.009 \mathrm{~h}$ and $t \simeq 0.2 \mathrm{~h}$. This behavior consists of a characteristic signature of a reservoir with formation damage $[10,32]$. After the flood front overcomes the damaged region, pressure derivative rises again and, hence, pressure increases. During falloff, however, it is not possible to identify the existence of skin effects from pressure derivative profile $[11,12]$.

Figs. 2.6 and 2.7 exhibit a comparison between the proposed two-phase model and the single-phase solution from Pan et al. [31], considering the reservoir properties of Table 2.1. Endpoint properties of oil and water were used to compute the single-phase pressure changes. During injection, pressure behavior is similar to the single-phase oil solution at early-times. As the flooded region propagates, pressure then detaches from the oil solution presents and intermediate behavior between the two presented single-phase solutions. The late-time constant derivative level during injection is slightly lower than the single-phase oil derivative. Eq. (2-31) evidences that pressure derivative at late-times is influenced by the flooded zone and, therefore, differs from the single-phase oil behavior.

During falloff, early-time pressure derivative reflects neither oil nor water 


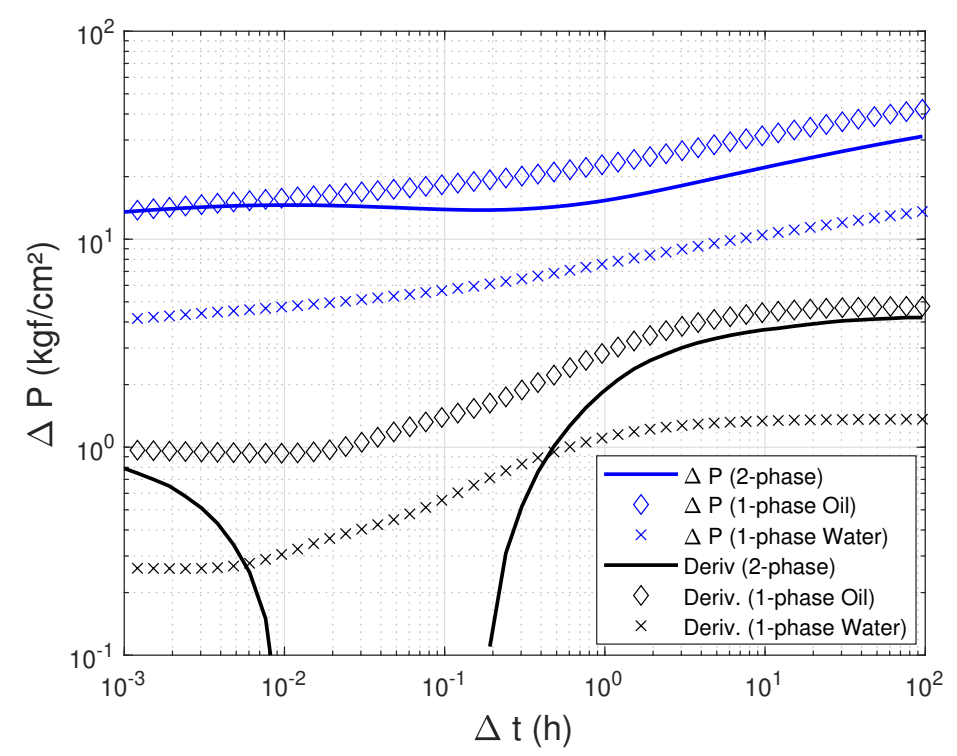

Figure 2.6: Comparison to single-phase responses for Case A during injection

properties, as evidenced in Figs. 2.6 and 2.7. Instead, it is influenced by the total mobility inside the swept area [11]. As flow-rate inside the flooded region tends to zero, then pressure becomes closer to the single-phase oil solution, which is more clearly noticed in the pressure derivative behavior.

Figs. 2.8 and 2.9 exhibit the results for injection and falloff periods considering the properties of Case B. This case aims to verify if the hypothesis that the zero-rate pulse equally propagates in all layers will hold in a 3-layered reservoir.

Again, the overall agreement between the analytical solutions and the

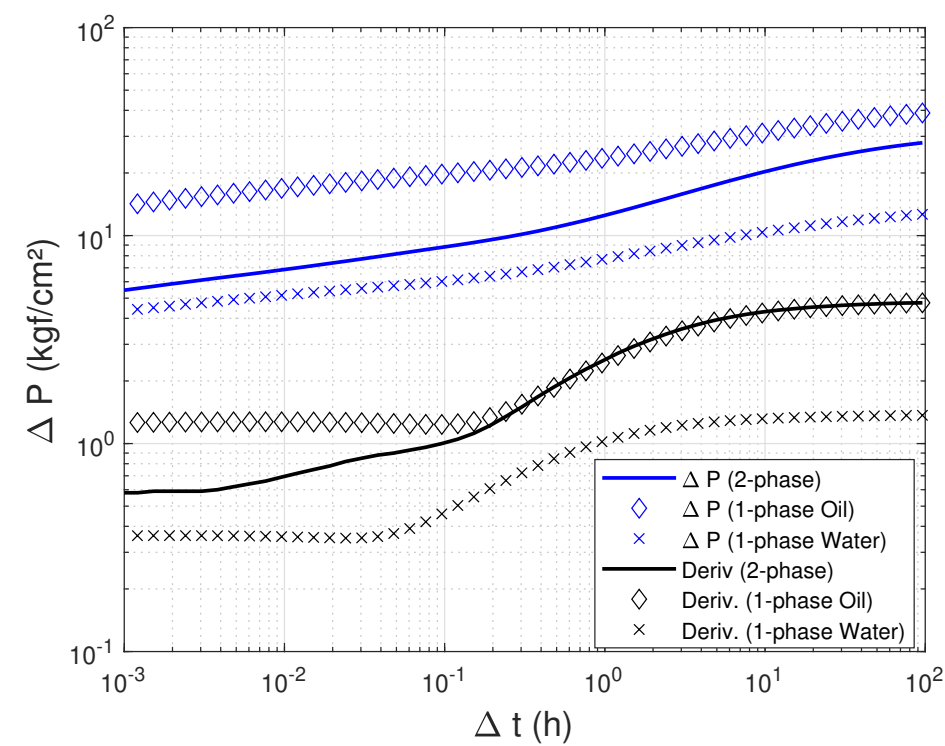

Figure 2.7: Comparison to single-phase responses for Case A during falloff 


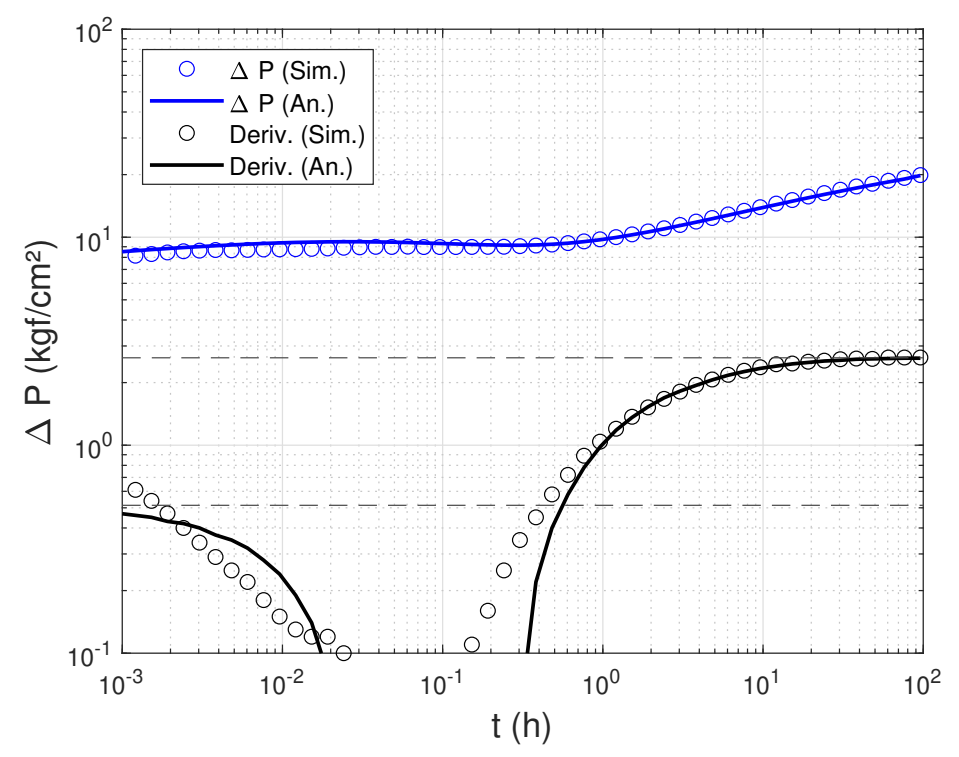

Figure 2.8: Pressure data for Case B during injection

numerical simulator is quite good, although some divergences were observed.

Flood front profile indicates that, once again, the "second radial - first radial" flow regime occurs at late-times during the injection period. Therefore, the theoretical pressure derivative level was determined from Eq. (2-31). Latetime derivative during falloff, in its turn, was computed from Eq. (2-35), while early-time derivative levels were evaluated using Eqs. (2-29) and (2-34).

The disagreement between analytical and numerical data for Case B is mainly explained by the same reasons detailed in the discussion of Case A: grid refinement and different skin zone representations. Moreover, the numerical

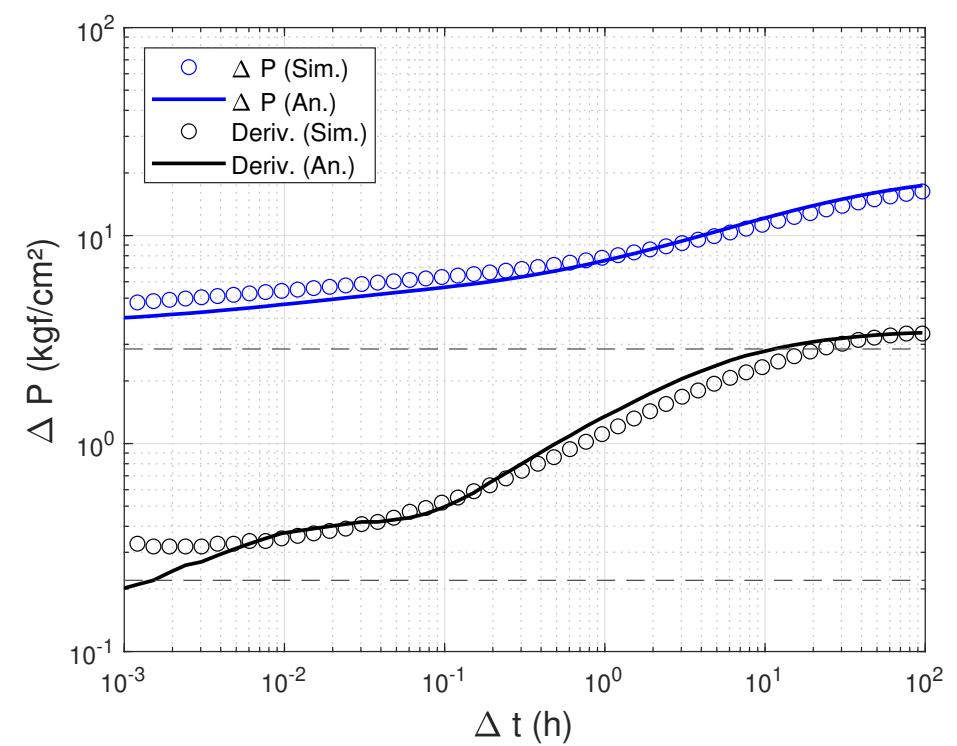

Figure 2.9: Pressure data for Case B during falloff 
simulator allows the zero-pulse rate to propagate differently in each layer, whereas the analytical solution assumes that this propagation is equal in all layers. This might explain the divergences observed in pressure derivative behavior during early falloff times. Nonetheless, numerical and analytical data converge after a shut-in time of around $0.004 \mathrm{~h}$ and a good agreement is observed until the end of the test. Grid refinement might also be related to this mismatch at early falloff times. Pressure derivative at early falloff times is slightly lower than the theoretical level foreseen by Eq. (2-34). Again, the reason for that is given by the injection time $t_{p}$, which was long enough so that the early radial flow had already ended at the moment of shut-in. Thus, Eq. $(2-34)$ is not valid.

Figs. 2.10 and 2.11 show the comparison between the two-phase solution and the single-phase pressure responses using oil and water endpoint properties. It is possible to notice that, similarly to Case A, pressure behavior at early injection times is close to the single-phase oil solution. Then, as the flooded region increases, pressure detaches from the single-phase oil solution. At late-time, pressure derivative stabilizes at a level slightly lower than the oil derivative, which is consistent with Eq. (2-31).

During falloff, pressure derivative starts approximately at the singlephase water level and quickly detaches from it. The increased wellbore length and absence of well off-centering in Case B possibly explain why a similar behavior was not identified in Case A. As flow-rate throughout the reservoir reaches zero, the two-phase falloff derivative converges to the single-phase oil solution.

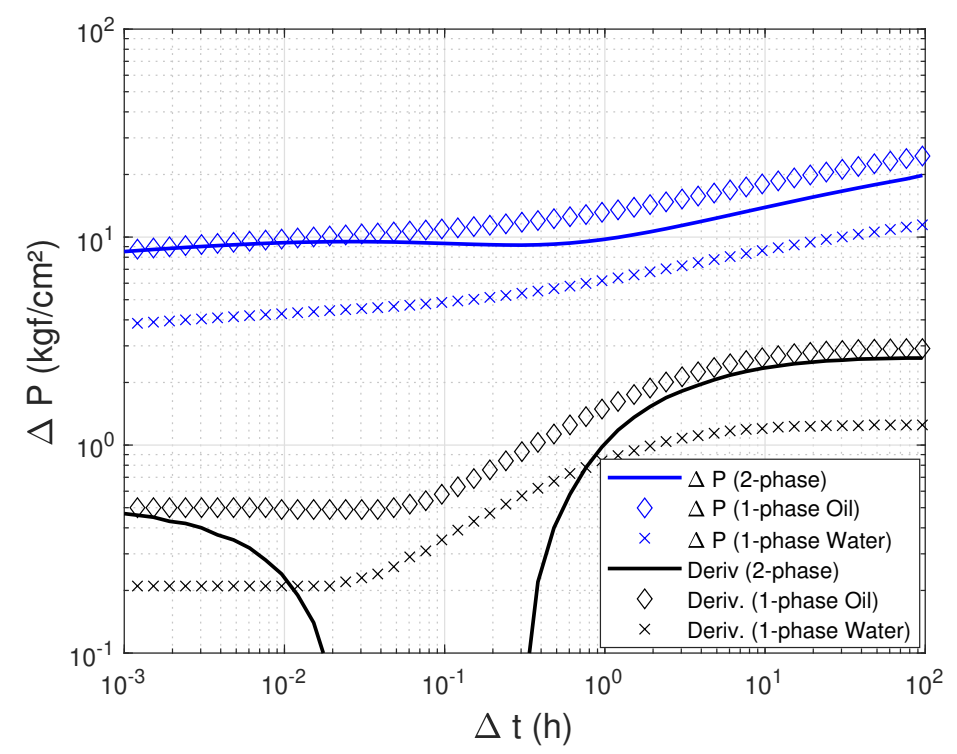

Figure 2.10: Comparison to single-phase responses for Case B during injection 


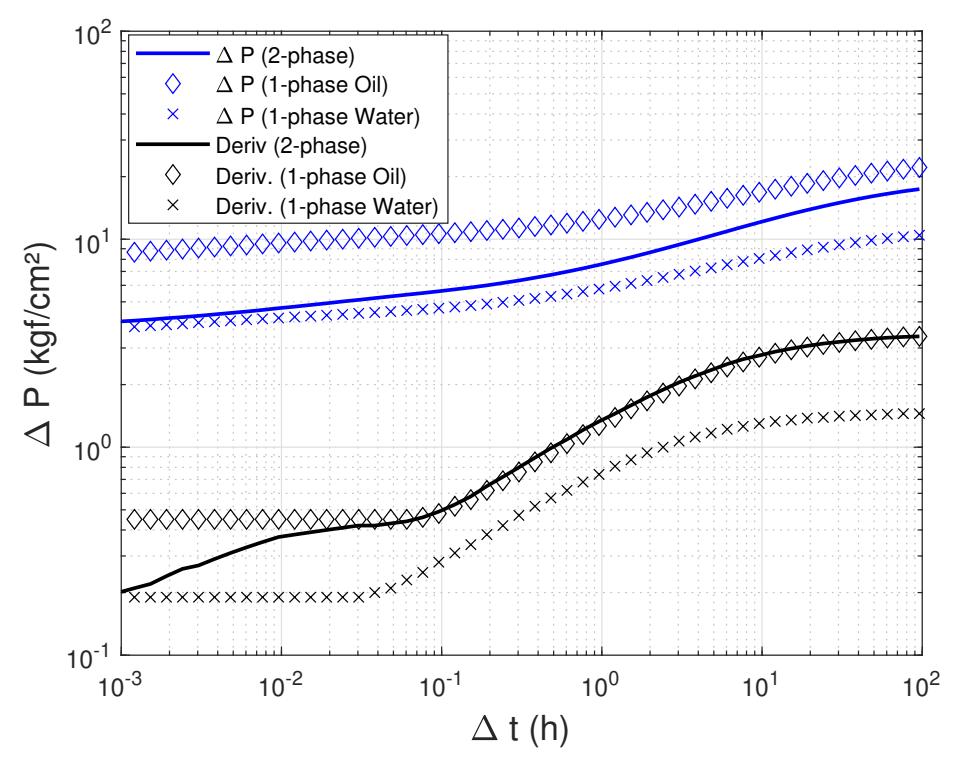

Figure 2.11: Comparison to single-phase responses for Case B during falloff

The results for Case $\mathrm{C}$ are exhibited in Figs. 2.12 and 2.13. This case presents the same properties as Case A, except for oil viscosity and skin zone parameters. The endpoint mobility ratio indicates that flow is favorable to oil. Thus, water saturation profile is not so smooth, and the numerical simulator presents some inherent issues due to the existence of shocks in the solution [16]. Therefore, numerical data present some instability during the injection period, which is more evidently perceived at the pressure derivative profile.

Despite that, pressure change curves present a good agreement. The overall derivative behavior during the injection period is also quite similar. Pressure derivative rises until a certain point, then a sharp drop is observed and, afterwards, the derivative stabilizes at a level, indicating that a late radial flow regime occurs. The blunt shift in pressure derivative that occurs around $t \simeq 0.2 \mathrm{~h}$ signs the moment when the flood front overcomes the skin zone radii [32]. Figs. 2.12 and 2.13 evidence that this derivative shift occurs at the same time for both analytical and numerical data. This endorses that the area equivalence used to define the skin zone radius in the analytical model was accurate.

During falloff, the flood front remains stationary. Thereby, the numerical instability issues are mitigated. For this reason, a better agreement between simulated and analytical data is observed. The detachment in pressure derivative is possibly related to the grid discretization. Nonetheless, analytical falloff solution fits the numerical data quite well.

The comparison between the two-phase solutions and the single-phase behavior computed using oil and water endpoint properties is exhibited in 


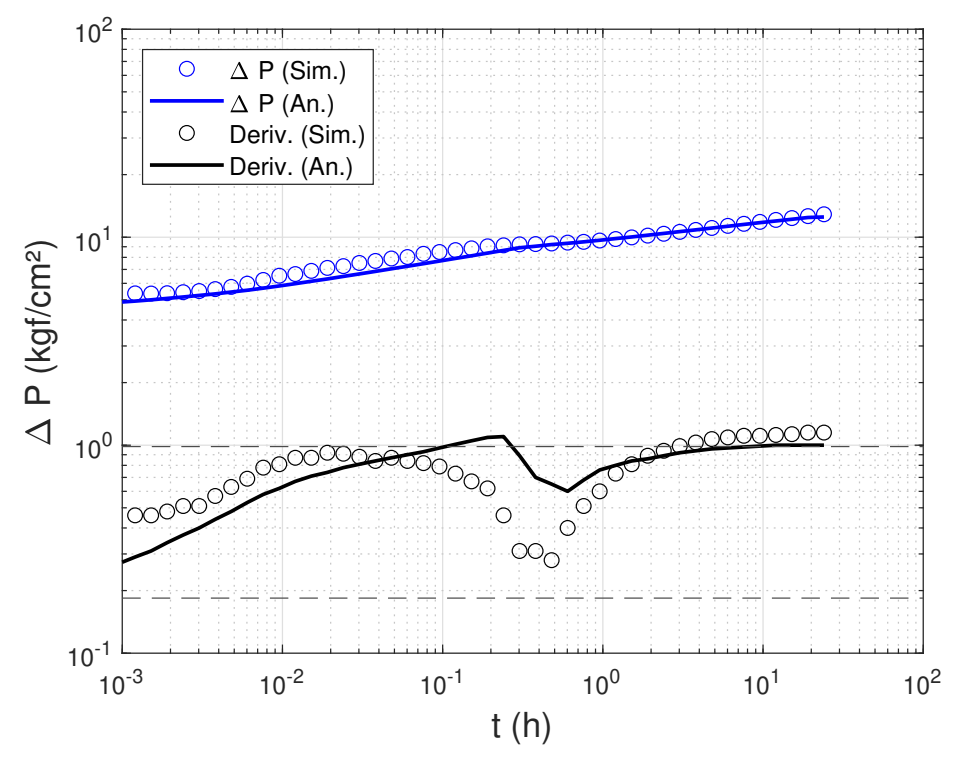

Figure 2.12: Pressure data for Case C during injection

Figs. 2.14 and 2.15. The mobility ratio in Case $\mathrm{C}$ is closer to unity than in

cases A and B. Hence, the single-phase responses from oil and water are not as far from each other as in the previous cases. Despite that, it is still possible to notice that pressure behavior during early injection times is similar to the single-phase oil solution. Then, as the flooded region increases, pressure change curve detaches from the oil solution.

Between $t \simeq 0.1$ and $t \simeq 0.3 \mathrm{~h}$, pressure change becomes close to the water solution. This behavior however, is explained by the flood front overcoming the skin zone, rather than a similarity between the two-phase and

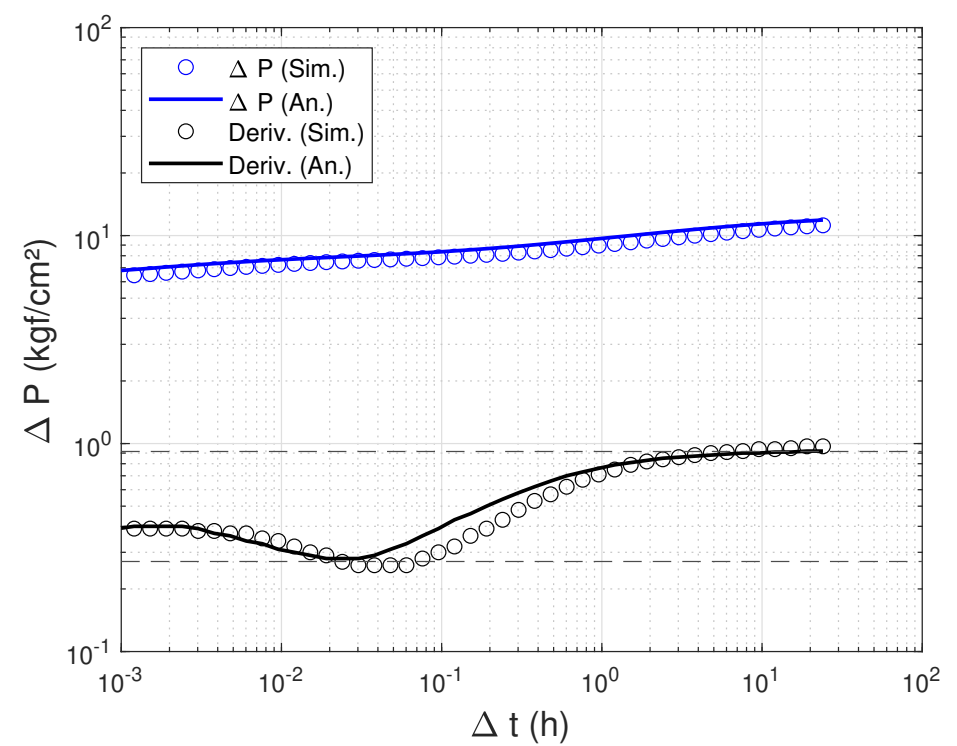

Figure 2.13: Pressure data for Case C during falloff 


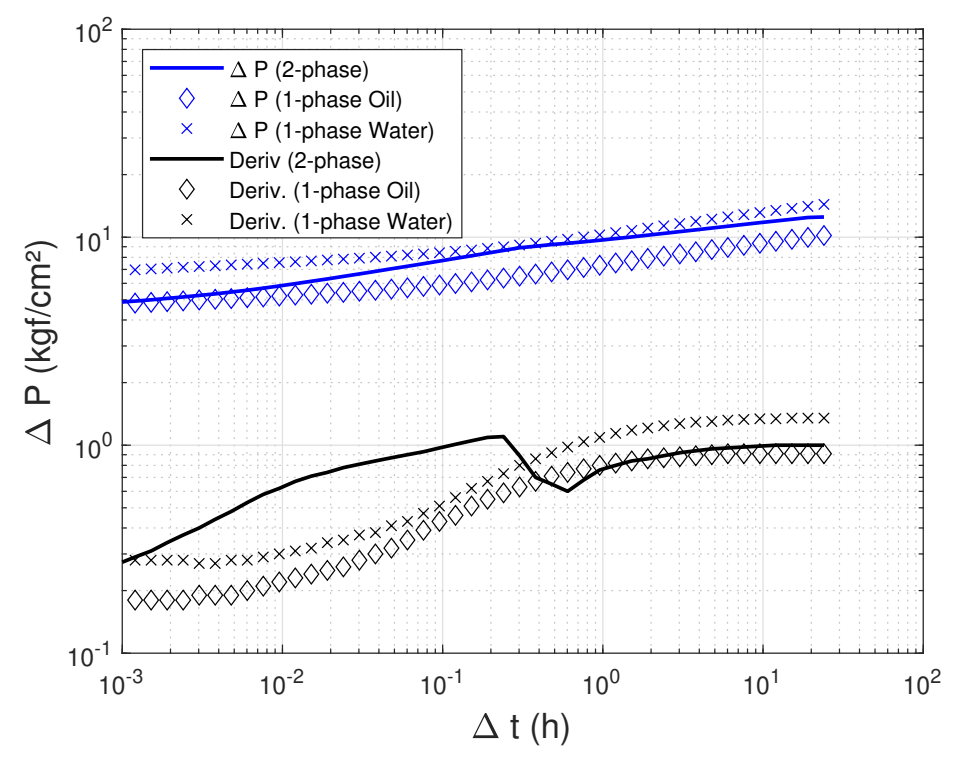

Figure 2.14: Comparison to single-phase responses for Case C during injection

the single-phase water solutions, as evidenced by pressure derivative.

During falloff, it is possible to identify that pressure change at earlytimes is close to the single-phase oil solution. Although pressure derivative in Case $\mathrm{C}$ remains at approximately equal to the single-phase water solution until around $\Delta t \simeq 0.003 \mathrm{~h}$, Eq. (2-34) is not applicable, since early radial flow during injection ended before the well was shut-in. Afterwards, as flow-rate inside the flooded region reaches zero, the derivative profile matches the single-phase oil solution. A similar behavior was observed in the previous cases.

The reservoir equivalent permeabilities were estimated from the constant

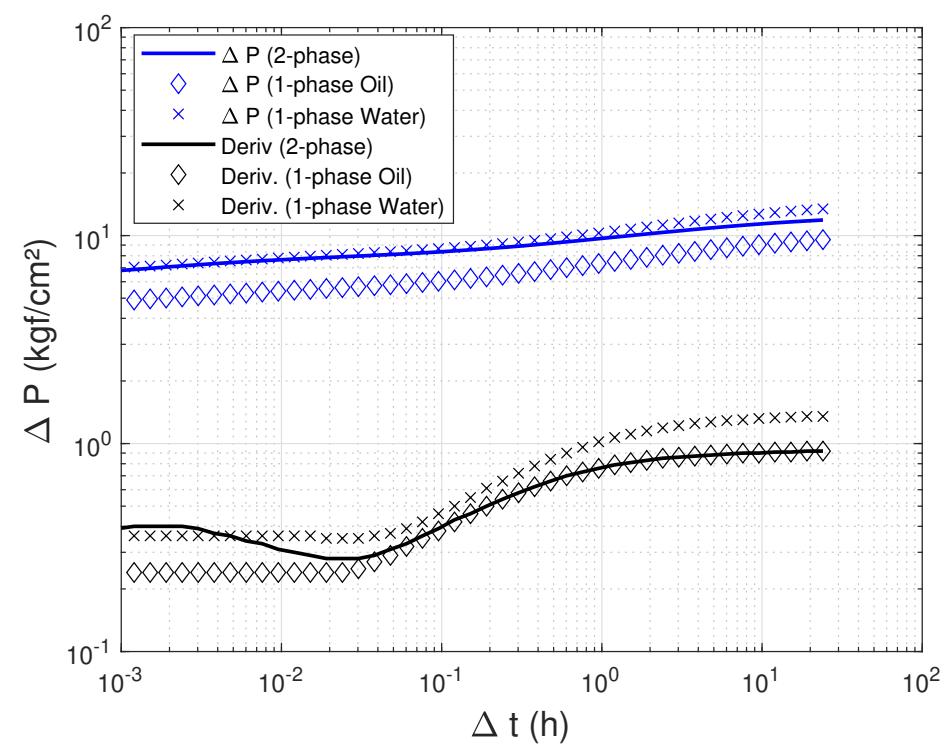

Figure 2.15: Comparison to single-phase responses for Case C during falloff 


\begin{tabular}{|c|c|c|c|c|}
\cline { 2 - 5 } \multicolumn{1}{c|}{} & Case & $\begin{array}{c}\left(k_{x y}\right)_{e q} \text { true } \\
(\mathrm{mD})\end{array}$ & $\begin{array}{c}\left(k_{x y}\right)_{e q} \text { est. } \\
(\mathrm{mD})\end{array}$ & $\begin{array}{c}\text { Error } \\
(\%)\end{array}$ \\
\hline $\begin{array}{c}\text { Injection } \\
\text { Falloff }\end{array}$ & $\mathrm{A}$ & 907 & 861 & -5.1 \\
869 & -4.2 \\
\hline $\begin{array}{c}\text { Injection } \\
\text { Falloff }\end{array}$ & \multirow{2}{*}{$\mathrm{B}$} & 783 & 753 & -3.8 \\
\hline $\begin{array}{c}\text { Injection } \\
\text { Falloff }\end{array}$ & $\mathrm{C}$ & \multirow{2}{*}{907} & 858 & 9.5 \\
\hline
\end{tabular}

Table 2.2: Estimated equivalent permeabilities

late-time pressure derivative levels. Results are reported in Table 2.2. Since the estimated permeabilities were computed from late-time data, they reflect the equivalent permeability in the horizontal plane. Permeabilities were computed by replacing the observed constant derivative level in equations (2-31) and $(2-35)$.

Early-time data were not used to evaluate the reservoir permeability because, for all cases, no constant derivative level was observed during injection and the early-time falloff derivative is not related to water or oil properties. In a real field test, however, early-time data may be affected by wellbore storage. Thus, it might be unfeasible to obtain the equivalent permeability in the $z x$ plane in a practical case.

Table 2.2 shows that the equivalent permeability could be computed with low error from both injection and falloff data. Nonetheless, it is important to notice that the application of Eq. (2-31) requires knowledge over the wellbore effective length. In a real field test, the total drilled length may not correspond to the total well length $[17,31]$. Thereby, in practice, falloff data provide a more reliable estimate for the reservoir equivalent permeability.

\section{5}

\section{Summary and Conclusions}

The main contribution of this Chapter is to present new analytical solutions for analyzing injectivity tests under two-phase oil-water flow conditions in multilayer reservoirs with multilateral horizontal wells, considering both injection and falloff periods. It was assumed no formation crossflow. The proposed formulation is based on the single-phase solution for multilateral wells achieved by Pan et al. [31] and the theory for injectivity tests in single-layer reservoirs developed by Peres and Reynolds [10] and Peres et al. [11].

The developed analytical model was achieved under some simplifying hypotheses. Some of those assumptions are quite common in the development of analytical solutions for well transient analysis, such as reservoir initially in 
equilibrium and slightly compressible fluids. On the other hand, some significantly strong assumptions were also made (e.g. negligible wellbore storage effects and stratified layers).

The developed formulations show that the same equations that depict pressure behavior in multilayer reservoirs with vertical wells $[12,14,16,32]$ are also applicable to horizontal wells, provided that the suitable adjustments are made. We have shown how these adjustments should be made.

The proposed solutions were verified by comparison to the results of a commercial flow simulator. Results showed that, although some divergences between the presented solution and the numerical simulator were observed, the overall agreement was good. This indicates that the hypotheses and approximations made are acceptable. The good match between analytical and numerical data during falloff also endorses the assumption originally made by Bela et al. [12] that the zero-rate pulse equally propagates in all layers.

Comparison with the single-phase oil and water solutions showed that, during injection, early-time pressure data presents a similar behavior to the single-phase oil solution. As the flood front propagates, pressure detaches from the single-phase oil solution. However, pressure derivative does not match the single-phase water level, unlike what happens in injectivity tests with vertical wells. During falloff, early-time data presents an intermediate behavior, between the single-phase oil and water solutions. Late-time falloff pressure derivative, collapses to the single-phase oil derivative, as flow-rate inside the flooded region becomes numerically equal to zero.

Moreover, early and late-time approximations for the theoretical early and late-time pressure derivative levels were proposed. While the early-time derivative level may not be identified, due to the wellbore and formation features, the reservoir horizontal equivalent permeability could be estimated with good accuracy. In a real field application, we recommend that the falloff derivative level should be used to estimate the permeability, as it does not require knowledge over the total effective drilled length. 


\section{3 \\ Impulse Functions Applied to Compute Pressure Change During Injectivity Tests}

This Chapter introduces an alternative formulation to evaluate pressure change during injectivity tests, based on Green's functions (or impulse functions). Section 3.1 motivates the development of this alternative solution and provides a brief literature overview about analytical solutions for two-phase flow. Section 3.2 details the proposed model to compute pressure change. The model validation is presented in Section 3.3, where the proposed formulation is compared to the previously existing solutions for pressure change during injectivity tests and to a commercial numerical flow simulator. Lastly, the main conclusions of this Chapter are found in Section 3.4.

\section{1 \\ Introduction}

Thompson and Reynolds [15] developed a solution for single-layered radially heterogeneous reservoirs. Their model has inspired the development of analytical solutions for pressure change during injectivity tests $[8,9,10,11$, $12,16,46]$.

Banerjee et al. [9] presented a formulation for injection/falloff tests in single-layer reservoirs with vertical wells. As outlined by Banerjee et al. [9], two-phase models for pressure change based on the Thompson and Reynolds' [15] theory assume that the rate-transient pulse propagates faster than the flood front. Hence, flow-rate inside the flooded region remains constant during the injection period. Fig. 3.1 shows a schematic representation to illustrate this assumption.

Peres and Reynolds [10] established objective criteria to verify that, in single-layer reservoirs, such condition holds for most cases of practical interest and also proposed a solution for injectivity tests in single-layer reservoirs with horizontal wells.

Boughara and Reynolds [17] included the thermal effects into the formulation and applied a non-linear regression method to estimate reservoir properties. Bonafé et al. [8] provided an approximate model for injectivity tests in single-layer reservoirs considering a multiple flow-rate scheme. 


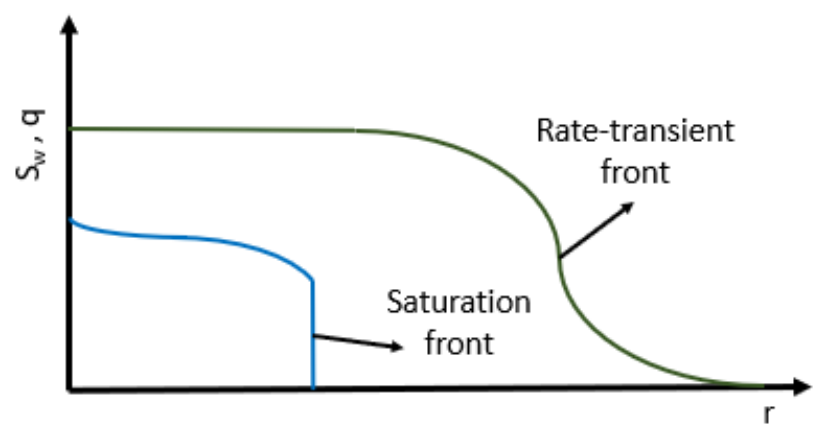

Figure 3.1: Schematics of rate transient front and water saturation front in a single-layer reservoir

Analytical solutions for injectivity tests in multilayer reservoirs $[12,16$, 46] were inspired by the single-layer solutions $[8,9,10,11,17]$ and, hence, by the Thompson and Reynolds' [15] steady-state theory. However, in multilayer reservoirs, layer flow-rates at the wellbore may change in time due to differences between layer properties $[3,5,14,16,32]$. This also includes the formulation presented in Section 2.3.2, which evidenced that layer flow-rates must be updated at each time step.

Fig. 3.2 exhibits an illustrative representation of layer flow-rate profiles at the wellbore and the respective rate transient fronts in a two-layered reservoir. As may be observed in Fig. 3.2, it is not possible to assure that flow-rate is constant throughout the flooded region in multilayer systems, due to the rate transient that occurs at the wellbore. Therefore, in a strict sense, the Thompson and Reynolds' [15] steady-state theory does not hold for multilayer reservoirs. This means, for instance, that flow-rate in layer $j$ may not be written out of the integral sign in Eq. (2-7), as it may change with the radius.

Neto et al. [47] proposed an alternative solution for injectivity tests in single-layer reservoirs. The flooded zone and the uninvaded region are described using a radially composite reservoir approach, where the interface between
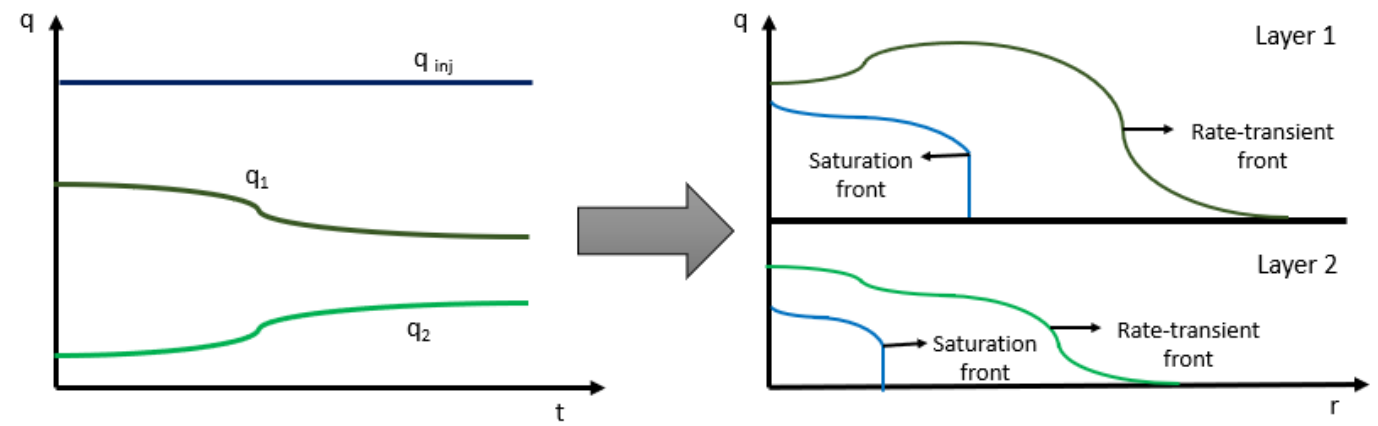

Figure 3.2: Layer flow-rate profiles at the wellbore (on the left) and rate and saturation fronts in each layer (on the right) 
regions (that is, the water front radius) moves in time [43]. The formulation presented by Neto et al. [47] does not require flow-rate within the flooded region to be constant. On the other hand, it assumes a piston-like fluid displacement.

Mastbaum et al. [48] also employed a radially heterogeneous reservoir model to develop a solution for multilayer stratified reservoirs, while Viana [49] included formation crossflow effects into the solution. The formulation proposed by Mastbaum et al. [48] and Viana [49] consists of solving, at each time step, a linear system to determine the pressure change. The order of this linear system progressively increases as the number of reservoir layers increases.

Green's functions (or impulse functions) for single-phase flow of slightly compressible fluids in single-layer reservoirs are known [50, 51]. Kuchuk and Wilkinson [52] developed an algorithm to determine the pressure response in commingled reservoirs using the appropriate single-layer impulse function for each layer. This algorithm to compute pressure change in stratified reservoirs was endorsed by Spath et al. [37], in a work that also depicted how layer flowrates at the wellbore may be computed. Lu et al. [53] applied Green's functions to develop a formulation for well tests in two-layer reservoirs with formation crossflow.

Thereby, solutions for conventional well testing based on Green's functions are already available. However, to the best of the author's knowledge, impulse functions have not yet been applied to evaluate the pressure change in reservoirs under two-phase flow, which is the case of injectivity tests.

Therefore, the main novelty of this Chapter is to obtain the impulse function for two-phase flow in single-layer reservoirs. A radially composite reservoir approach was employed to account for the distinct fluids (oil and water) that flow throughout the reservoir during an injectivity test. The singlelayer solution may be coupled to the algorithm presented by Kuchuk and Wilkinson [52] and Spath et al. [37] to obtain pressure and flow-rate profiles in multilayer reservoirs. Additionally, an approximate solution for pressure change during injectivity tests in single-layer reservoirs with horizontal wells is derived by applying Newman's product $[54,55]$ and the presented single-layer formulation. In this work, we focus only on the injection period, that is, the falloff period was not considered.

The proposed models were validated via comparison, for a set of synthetic cases, to a commercial finite difference-based flow simulator and to the previously existing solutions $[10,16,48]$. We also provide a brief discussion regarding the radial flow regime that may be observed during an injectivity test. Furthermore, the Delta Transient Method [56, 57] was applied to estimate layer permeabilities in the multilayer cases. 


\section{2 \\ Proposed Formulation}

This Section outlines the proposed formulation for injectivity tests using impulse functions. The reservoir is assumed to be homogeneous, laterally infinite and initially in equilibrium. Fluids are considered to be slightly compressible, with constant viscosity and compressibility. The presented formulation considers that capillary and gravitational forces are negligible. An isothermal flow is considered and a piston-like fluid displacement is assumed. Finally, all computations were made assuming a consistent set of units.

\subsection{1}

\section{Impulse Function for Single-Layer Reservoirs with Vertical Wells}

In this Section, the flooded region resulting from water injection is represented using a radially composite approach. Water injection in a single-layer reservoir, then, may be understood as a problem of a radially heterogeneous reservoir with a moving boundary $r_{F}(t)[43,48,49]$. Formation damage is included into the model by defining an annular region, concentric to the wellbore axis, where the permeability $k_{s}$ is different than the reservoir permeability $k$ $[47,58]$. Wellbore storage will be neglected in a first moment and incorporated into the model at the end of this Section.

Figs. 3.3 and 3.4 show a schematic representation of the reservoir top and lateral view at two distinct stages: while the water front is within the damaged region and after the flooded region has overcome the skin radius, respectively. In Figs. 3.3 and 3.4, the skin radius $r_{s k i n}$ is represented as $r_{s}$ for convenience, while $r_{w}$ denotes the wellbore radius, $r_{F}$ denotes the water front radius and $r_{e}$ denotes the reservoir outer radius.

The water front radius at each time step is determined according to
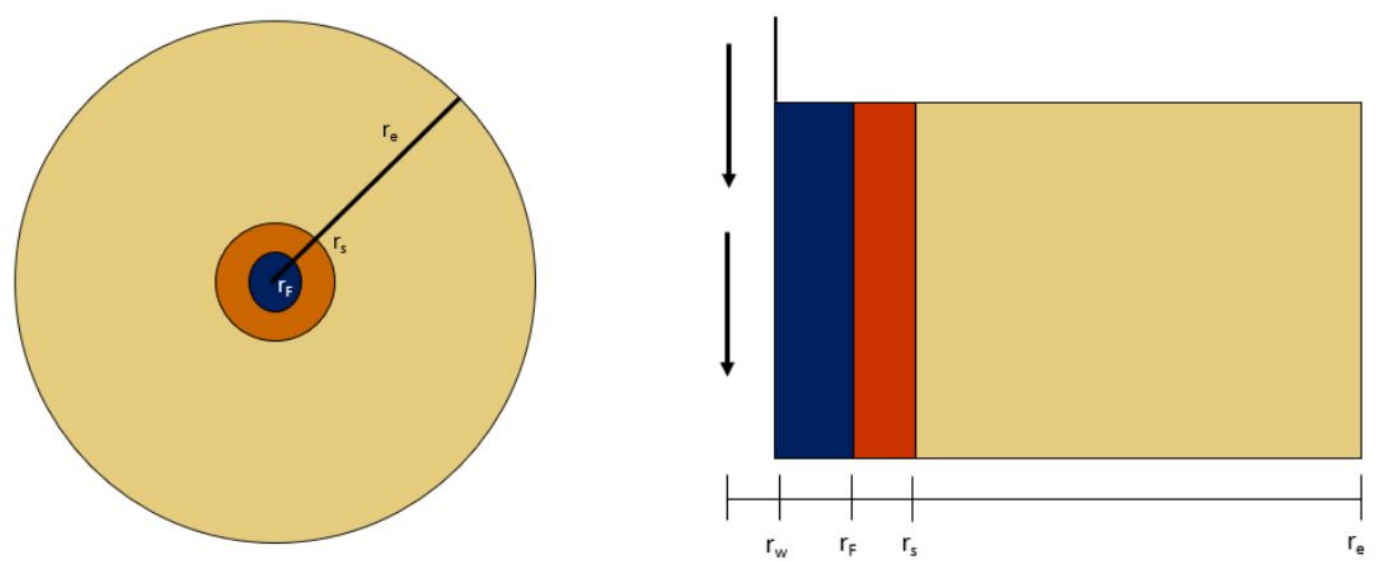

Figure 3.3: Reservoir top (on the left) and lateral views for $r_{F}<r_{\text {skin }}$. 

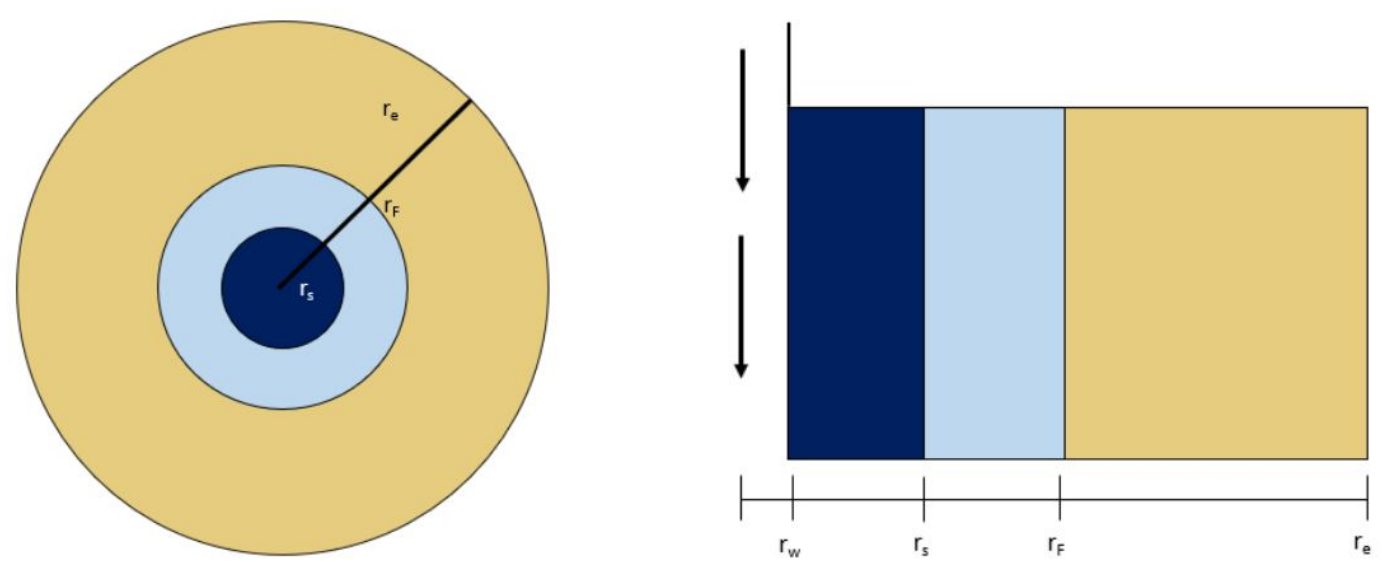

Figure 3.4: Reservoir top (on the left) and lateral views for $r_{F}>r_{s k i n}$.

the Buckley-Leverett [40] theory, using Eq. (2-3). Since a piston-like fluid displacement is considered in this Chapter, the fractional flow derivative $f_{w}^{\prime}$ is given by:

$$
f_{w}^{\prime}=\frac{1}{1-S_{w i}-S_{o r}}
$$

The reservoir, then, may be split into three distinct regions, depending on the flood front position. While the water front is within the damaged zone:

Region 1: goes from $r=r_{w}$ to $r=r_{F}(t)$

Region 2: goes from $r=r_{F}(t)$ to $r=r_{\text {skin }}$

Region 3: goes from $r=r_{\text {skin }}$ to the reservoir outer radius

And after the water front has overcome the skin zone:

Region 1: goes from $r=r_{w}$ to $r=r_{\text {skin }}$

Region 2: goes from $r=r_{\text {skin }}$ to $r=r_{F}(t)$

Region 3: goes from $r=r_{F}(t)$ to the reservoir outer radius

According to the model assumptions mentioned above, flow in porous media obeys the diffusivity equation given by $[5,58]$ :

PDE:

$$
\frac{1}{r} \frac{\partial}{\partial r}\left(r \frac{\partial \Delta p_{i}}{\partial r}\right)-\frac{1}{\eta_{i}} \frac{\partial \Delta p_{i}}{\partial t}=0
$$

where the subscript $i \in\{1,2,3\}$ indexes the region, $r$ stands for the radius, $t$ denotes the time and $\Delta p=p(r, t)-p(r, t=0)$ indicates the pressure change 
resulting from the water injection. The hydraulic diffusivity $\eta_{i}$ is defined as $[3,53]$ :

$$
\eta_{i}=\frac{k_{i} \lambda_{i}}{\phi_{i} c_{t}}
$$

where $\lambda_{i}=k_{r i} / \mu_{i}$ represents the fluid mobility $[10,12,14,16]$, considering the suitable fluid that flows in region $i$; that is, oil or water.

The initial condition, inner and outer boundary conditions for the problem are given by:

Initial Condition (IC):

$$
\Delta p_{i}(r, t=0)=0 .
$$

Inner Boundary Condition (IBC):

$$
\left.2 \pi h k_{1} \lambda_{1}\left(r \frac{\partial \Delta p_{1}}{\partial r}\right)\right|_{r \rightarrow r_{w}}=\delta(t)
$$

where $h$ stands for the reservoir thickness and $\delta$ denotes the Dirac delta function. The IBC defined in Eq. (3-5) represents an instant injection of a unitary volume of water at $t=0$.

Outer Boundary Condition (OBC):

$$
\lim _{r_{e} \rightarrow \infty} \Delta p_{3}\left(r=r_{e}, t\right)=0 .
$$

Eq. (3-6) represents the outer boundary condition for a laterally infinite reservoir $[5,12,20,47]$. The proposed formulation is also applicable for closed boundary or constant pressure condition at the reservoir outer boundary, provided that Eq. (3-6) is replaced by the suitable boundary condition.

Note that the problem defined by Eqs. (3-2) to (3-6) remains ill-posed so far, as the PDE presented in Eq. (3-2) demands two spatial conditions per region. Since the radially heterogeneous reservoir model consists of three distinct regions, four spatial conditions are still needed to obtain a wellposed problem. The missing spatial conditions are obtained from the pressure continuity and mass conservation at the interface between regions, yielding coupling conditions between regions (or CCRs) [24, 47, 48, 49, 58]:

Pressure CCR:

$$
\lim _{r \rightarrow r_{i}^{-}} \Delta p_{i}(r, t)=\lim _{r \rightarrow r_{i}^{+}} \Delta p_{i+1}(r, t) .
$$

Flow-rate CCR:

$$
\lim _{r \rightarrow r_{i}^{-}} k_{i} \lambda_{i}\left(r \frac{\partial \Delta p_{i}(r, t)}{\partial r}\right)=\lim _{r \rightarrow r_{i}^{+}} k_{i+1} \lambda_{i+1}\left(r \frac{\partial \Delta p_{i+1}(r, t)}{\partial r}\right),
$$


where $i \in\{1,2\}$ in Eqs. (3-7) and (3-8) indexes the region. Eqs. (3-7) and (3-8) are required to assure that pressure and flow-rate profiles along the reservoir are continuous.

Applying Laplace transform to the problem defined by Eqs. (3-2) to (3-8), the following ODE is obtained:

$$
\frac{1}{r} \frac{\partial}{\partial r}\left(r \frac{\partial \overline{\Delta p}_{i}}{\partial r}\right)-\frac{u \overline{\Delta p}_{i}}{\eta_{i}}=0, \quad \text { for } i \in\{1,2,3\}
$$

where $u$ denotes the Laplace variable.

The general solution of Eq. (3-9) is given by [5, 20, 47, 49, 59]:

$$
\overline{\Delta p}_{i}(r, u)=a_{i} I_{0}\left(r \sqrt{\frac{u}{\eta_{i}}}\right)+b_{i} K_{0}\left(r \sqrt{\frac{u}{\eta_{i}}}\right) .
$$

Thus, taking the derivative of Eq. (3-10) with respect to the radius [60]:

$$
\frac{\partial \overline{\Delta p}_{i}}{\partial r}(r, u)=a_{i} \sqrt{\frac{u}{\eta_{i}}} I_{1}\left(r \sqrt{\frac{u}{\eta_{i}}}\right)-b_{i} \sqrt{\frac{u}{\eta_{i}}} K_{1}\left(r \sqrt{\frac{u}{\eta_{i}}}\right) .
$$

Relations between the coefficients $a_{i}$ and $b_{i}$ in each region may be obtained from the boundary and coupling conditions. The outer boundary condition defined in Eq. (3-6) implies that $a_{3}=0$, due to the limits of the modified Bessel's functions when the argument goes to infinity [5, 47, 60].

From Eqs. (3-5) and (3-11) and the Laplace transform of the Dirac delta function [60], the IBC in Laplace domain may be written as:

$$
a_{1} I_{1}\left(r_{w} \sqrt{\frac{u}{\eta_{1}}}\right)-b_{1} K_{1}\left(r_{w} \sqrt{\frac{u}{\eta_{1}}}\right)=\frac{1}{r_{w}} \sqrt{\frac{\eta_{1}}{u}} \frac{1}{2 \pi h k_{1} \lambda_{1}} .
$$

From Eq. (3-10) and the CCRs defined in Eq. (3-7), pressure equality at the interface between regions implies that:

$$
a_{i} I_{0}\left(r_{i} \sqrt{\frac{u}{\eta_{i}}}\right)+b_{i} K_{0}\left(r_{i} \sqrt{\frac{u}{\eta_{i}}}\right)-a_{i+1} I_{0}\left(r_{i} \sqrt{\frac{u}{\eta_{i+1}}}\right)-b_{i+1} K_{0}\left(r_{i} \sqrt{\frac{u}{\eta_{i+1}}}\right)=0
$$

for $i \in\{1,2\}$.

Finally, from Eqs. (3-10) and (3-8), mass conservation at the interface between regions is expressed as:

$$
\begin{aligned}
& a_{i} \frac{u k_{i} \lambda_{i}}{\sqrt{\eta_{i}}} I_{1}\left(r_{i} \sqrt{\frac{u}{\eta_{i}}}\right)-b_{i} \frac{u k_{i} \lambda_{i}}{\sqrt{\eta_{i}}} K_{1}\left(r_{i} \sqrt{\frac{u}{\eta_{i}}}\right)- \\
& \quad-a_{i+1} \frac{u k_{i+1} \lambda_{i+1}}{\sqrt{\eta_{i+1}}} I_{1}\left(r_{i} \sqrt{\frac{u}{\eta_{i+1}}}\right)+b_{i+1} \frac{u k_{i+1} \lambda_{i+1}}{\sqrt{\eta_{i+1}}} K_{1}\left(r_{i} \sqrt{\frac{u}{\eta_{i+1}}}\right)=0 .
\end{aligned}
$$

Rearranging Eq. (3-14): 


$$
\begin{aligned}
& a_{i} I_{1}\left(r_{i} \sqrt{\frac{u}{\eta_{i}}}\right)-b_{i} K_{1}\left(r_{i} \sqrt{\frac{u}{\eta_{i}}}\right)+ \\
& \quad+\underbrace{\frac{k_{i+1} \lambda_{i+1}}{k_{i} \lambda_{i}} \sqrt{\frac{\eta_{i}}{\eta_{i+1}}}}_{=\gamma_{i}}\left[-a_{i+1} I_{1}\left(r_{i} \sqrt{\frac{u}{\eta_{i+1}}}\right)+b_{i+1} K_{1}\left(r_{i} \sqrt{\frac{u}{\eta_{i+1}}}\right)\right]=0
\end{aligned}
$$

for $i \in\{1,2\}$.

Writing Eqs. (3-12), (3-13) and (3-15) as a linear system:

$$
[M]\left[\begin{array}{c}
a_{1} \\
b_{1} \\
a_{2} \\
b_{2} \\
b_{3}
\end{array}\right]=\left[\begin{array}{c}
\frac{1}{r_{w}} \sqrt{\frac{\eta_{1}}{u}} \frac{1}{2 \pi h k_{1} \lambda_{1}} \\
0 \\
0 \\
0 \\
0
\end{array}\right]
$$

where matrix $M$ is defined as:

$$
M=\left[\begin{array}{ccccc}
I_{1}\left(r_{w} \sqrt{\frac{u}{\eta_{1}}}\right) & -K_{1}\left(r_{w} \sqrt{\frac{u}{\eta_{1}}}\right) & 0 & 0 & 0 \\
I_{0}\left(r_{1} \sqrt{\frac{u}{\eta_{1}}}\right) & K_{0}\left(r_{1} \sqrt{\frac{u}{\eta_{1}}}\right) & -I_{0}\left(r_{1} \sqrt{\frac{u}{\eta_{2}}}\right) & -K_{0}\left(r_{1} \sqrt{\frac{u}{\eta_{2}}}\right) & 0 \\
I_{1}\left(r_{1} \sqrt{\frac{u}{\eta_{1}}}\right) & -K_{1}\left(r_{1} \sqrt{\frac{u}{\eta_{1}}}\right) & -\gamma_{1} I_{1}\left(r_{1} \sqrt{\frac{u}{\eta_{2}}}\right) & \gamma_{1} K_{1}\left(r_{1} \sqrt{\frac{u}{\eta_{2}}}\right) & 0 \\
0 & 0 & I_{0}\left(r_{2} \sqrt{\frac{u}{\eta_{2}}}\right) & K_{0}\left(r_{2} \sqrt{\frac{u}{\eta_{2}}}\right) & -K_{0}\left(r_{2} \sqrt{\frac{u}{\eta_{3}}}\right) \\
0 & 0 & I_{1}\left(r_{2} \sqrt{\frac{u}{\eta_{2}}}\right) & -K_{1}\left(r_{2} \sqrt{\frac{u}{\eta_{2}}}\right) & \gamma_{2} K_{1}\left(r_{2} \sqrt{\frac{u}{\eta_{3}}}\right)
\end{array}\right],
$$

where:

$$
\gamma_{i}=\frac{k_{i+1} \lambda_{i+1}}{k_{i} \lambda_{i}} \sqrt{\frac{\eta_{i}}{\eta_{i+1}}} .
$$

Solving the linear system defined in Eq. (3-16), the coefficients $a_{i}$ and $b_{i}$ in each region may be determined. Thereby, pressure change at the wellbore can be evaluated as:

$$
\bar{G}_{w}(u)=a_{1} I_{0}\left(r_{w} \sqrt{\frac{u}{\eta_{1}}}\right)+b_{1} K_{0}\left(r_{w} \sqrt{\frac{u}{\eta_{1}}}\right) .
$$

Eq. (3-19) represents the pressure response measured at the wellbore due to the instant injection of a unitary volume of water into the reservoir at $t=0$, as defined in the IBC given by Eq. (3-5). Thus, the term $\bar{G}_{w}$ denotes the impulse function (or Green's function) in Laplace domain [50, 55], as a consequence of an instantaneous water injection of unitary volume. Note that the impulse function at the wellbore must be evaluated using the properties corresponding to Region 1, which is the region that contains the well. Thus, pressure change during an injectivity test may be determined as $[50,59,63]$ :

$$
\overline{\Delta p}_{w f}(u)=\bar{q}_{i n j}(u) \bar{G}_{w}(u) .
$$




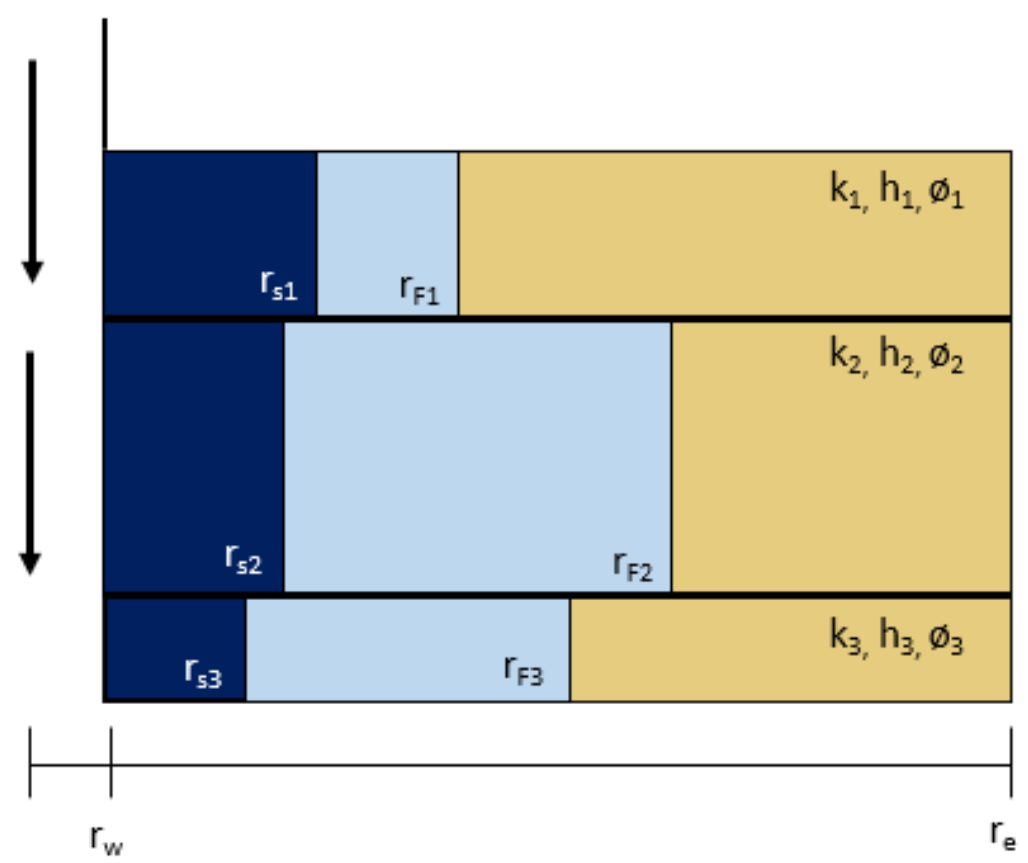

Figure 3.5: Schematics of a multilayer reservoir with vertical injector well

Finally, wellbore storage effects may be easily incorporated into Eq. (3-20) using the following relation $[5,22,37,63]$ :

$$
\overline{\Delta p}_{w f}(u, C)=\frac{\overline{\Delta p}_{w f}(u)}{1+u^{2} C \overline{\Delta p}_{w f}(u)},
$$

where $C$ stands for the storage coefficient.

\subsection{2}

\section{Extension to Multilayer Reservoirs}

Now, consider a reservoir with $n$ distinct layers, such as portrayed in Fig. 3.5, which shows an schematic representation for a given time when the flood front has already overcome the damaged region in all layers. Each layer is homogeneous and may present distinct properties, such as permeability, porosity and thickness. Skin zone properties may also be different in each layer. Besides, pressure change is assumed to be the same in all layers (apart from the hydrostatic effect).

Applying Eq. (3-20) into a given reservoir layer $j[37,52]$ :

$$
\overline{\Delta p}_{w f j}(u)=\bar{q}_{j}(u) \bar{G}_{w, j}(u) .
$$

By model hypothesis, pressure change at the wellbore is the same in all layers, apart from the hydrostatic effect. Therefore [12, 16, 30, 31]:

$$
\Delta p_{w f 1}(t)=\Delta p_{w f 2}(t)=\cdots=\Delta p_{w f n}(t)=\Delta p_{w f}(t)
$$


Thus, from Eqs. (3-22) and (3-23), flow-rate in layer $j$ is given by:

$$
\bar{q}_{j}=\frac{\overline{\Delta p}_{w f}}{\bar{G}_{w, j}} .
$$

Summing Eq. (3-24) for all layers:

$$
\sum_{j=1}^{n} \bar{q}_{j}=\overline{\Delta p}_{w f} \sum_{j=1}^{n} \frac{1}{\bar{G}_{w, j}} .
$$

Considering that the injection flow-rate $q_{i n j}$ is constant during the test, mass conservation inside the wellbore implies that $[16,31]$ :

$$
\sum_{j=1}^{n} q_{j}(t)=q_{i n j} \quad \Longrightarrow \quad \sum_{j=1}^{n} \bar{q}_{j}=\frac{q_{i n j}}{u} .
$$

Hence, from Eqs. (3-25) and (3-26):

$$
\overline{\Delta p}_{w f}=\frac{q_{i n j}}{u} \sum_{j=1}^{n}\left(\frac{1}{\bar{G}_{w, j}}\right)^{-1} .
$$

Eq. (3-27) was proposed by Spath et al. [37] to determine the pressure change in multilayer reservoirs. This formulation was originally developed by Kuchuk and Wilkinson [52]. Unlike the work presented in this Chapter, Kuchuk and Wilkinson [52] also accounted for an eventual difference between initial pressures in each layer.

Then, a computational implementation of the formulation detailed in this Section may be developed based on the flowchart exhibited in Fig. 3.6.

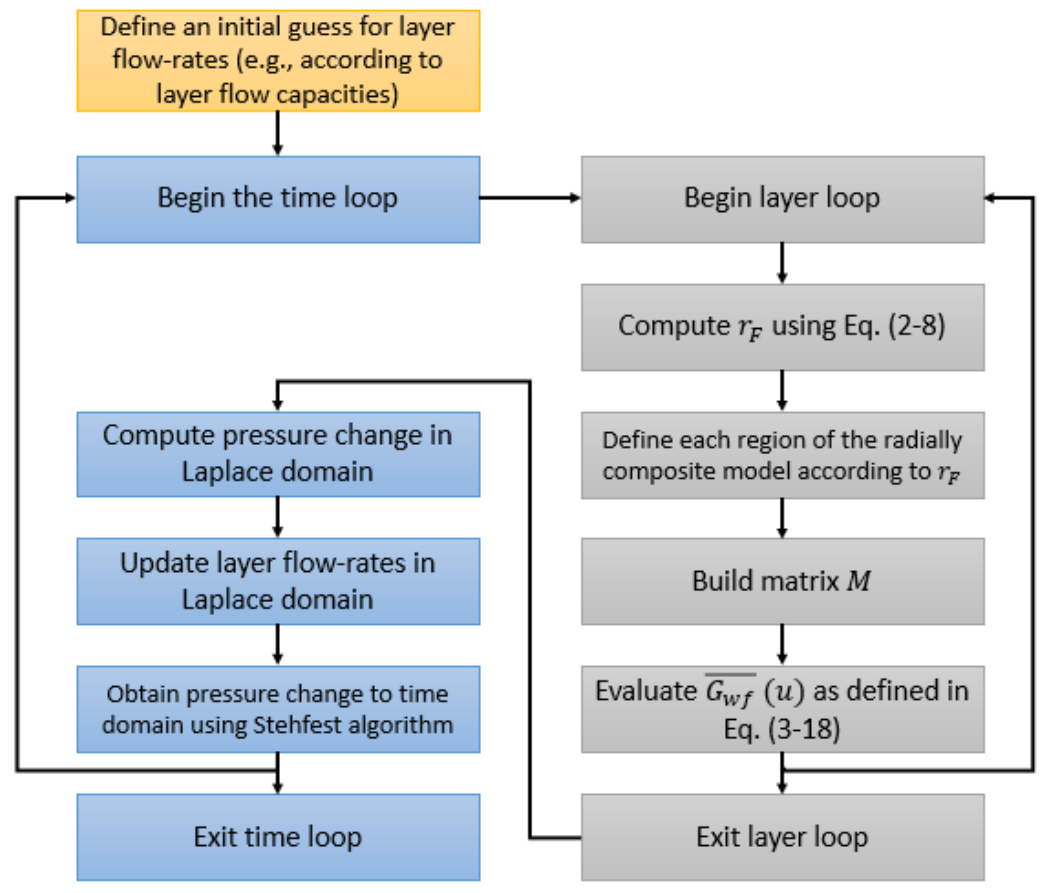

Figure 3.6: Flowchart to implement the proposed formulation 


\subsection{3}

\section{Application to Single-Layer Reservoirs with Horizontal Wells}

In reservoirs with horizontal wells, flow in each coordinate axis affects the pressure change behavior. The problem, then, is modeled by the following PDE [6, 30]:

$$
\frac{\partial^{2} \Delta p}{\partial x^{2}}+\frac{\partial^{2} \Delta p}{\partial y^{2}}+\frac{\partial^{2} \Delta p}{\partial z^{2}}-\frac{1}{\eta} \frac{\partial \Delta p}{\partial t}=0
$$

There already exist analytical models for single-phase flow in single-layer reservoirs with horizontal wells [4, 6, 21, 33]. For a uniform influx wellbore, the solution in real time domain is obtained by applying Newman's [54, 55] product and impulse functions [18, 22, 50]:

$$
\Delta p(x, y, z, t)=q \int_{0}^{t} \frac{\Delta p_{x}(x, \tau)}{L \phi c_{t}} \Delta p_{y}(y, \tau) \Delta p_{z}(z, \tau) d \tau .
$$

where $\Delta p_{x}, \Delta p_{y}$ and $\Delta p_{z}$ denote the respective impulse functions in $x-, y$ - and $z$-directions. This formulation is detailed in Appendix A.

When it comes to water injection in single-layer reservoirs with horizontal wells, an analytical model was developed by Peres and Reynolds [10]. Their formulation considers that pressure change at the wellbore may be computed as the sum of two terms: one that comes from the single-phase oil solution and another that accounts for the mobility differences within the flooded region, considering the flooding patterns proposed by Deppe [41]. Their formulation was presented in Section 2.3.1.

During injectivity tests with horizontal wells, the flood front initially propagates in the vertical plane perpendicular to the wellbore axis, as explained by Peres and Reynolds [10] and Peres et al. [11]. This effect was also commented in Sections 2.3.1 to 2.3.3.

Therefore, this Section proposes to approximate an injectivity test in a

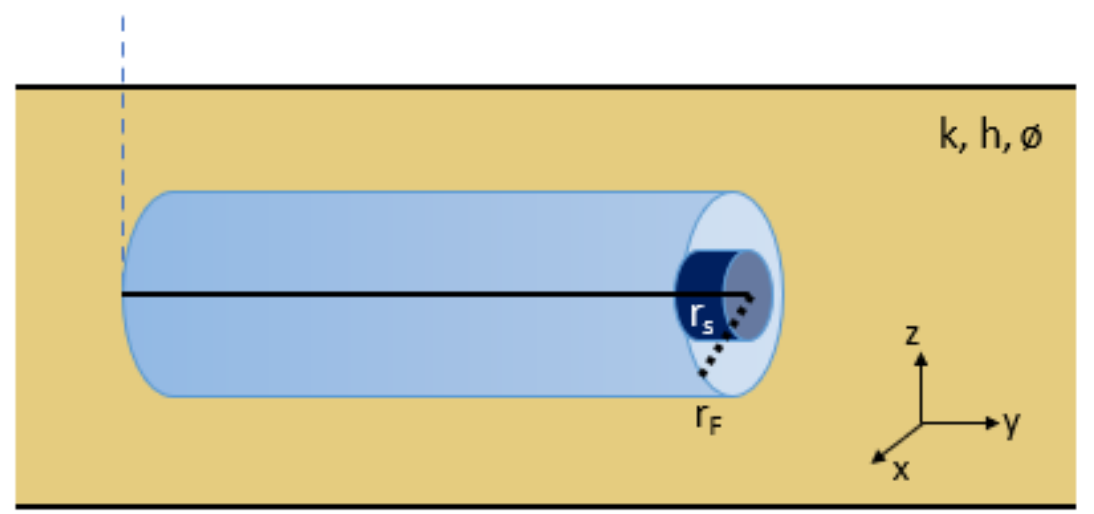

Figure 3.7: Horizontal injector well model (lateral view) 


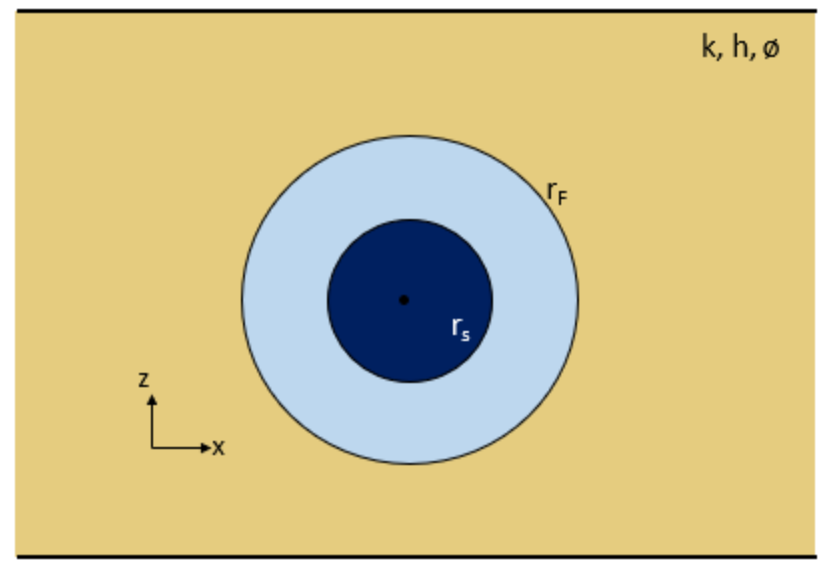

Figure 3.8: Horizontal injector well model (view of the $z x$-plane)

single-layer reservoir with horizontal well by a radially heterogeneous reservoir model. The reservoir is assumed to be isotropic, that is, $k_{x}=k_{y}=k_{z}=k$. Fig. 3.7 shows a schematics of a single-layer reservoir with a horizontal injector well, for a given moment when the flood front is beyond the damaged region and assuming that the wellbore is parallel to the $y$-axis. Fig. 3.8 exhibits the view in $z x$-plane of this reservoir model.

As evidenced in Fig. 3.7, the approximated radially heterogeneous model for injectivity tests with horizontal wells considers that the flood front propagates only along the $z x$-plane. That is, water saturation beyond the wellbore tips is assumed to remain constant and equal to the initial water saturation $S_{w i}$. This assumption, albeit unrealistic, is expected to be reasonable, considering that the wellbore length is typically much longer compared to the reservoir thickness. Besides, water flow beyond the wellbore tips at early-times is also unaccounted for in the model proposed by Peres and Reynolds [10].

One should notice, however, that waterfront propagation in horizontal plane becomes increasingly more relevant as the injection goes on [10, 41]. Thus, the radially heterogeneous model exhibited in Figs. 3.7 and 3.8 is valid only in an approximate sense, as it does not accurately describe the flood front propagation.

On the other hand, pressure transient front does not depend on the flood front position. Hence, considering the notation defined by Peres and Reynolds [10] that accounts for both pressure transient front and water front, the approximate radially heterogeneous reservoir model shown in Figs. 3.7 and 3.8 is applicable for the "first radial - first radial", "first linear - first radial" and "second radial - first radial" flow-regimes.

Under those assumptions, and defining $r^{2}=x^{2}+z^{2}$, pressure change during injectivity tests in single-layer reservoirs with horizontal wells is governed 
by the following PDE:

$$
\frac{\partial^{2} \Delta p_{i}}{\partial y^{2}}+\frac{1}{r} \frac{\partial}{\partial r}\left(r \frac{\partial \Delta p_{i}}{\partial r}\right)-\frac{1}{\eta_{i}} \frac{\partial \Delta p_{i}}{\partial t}=0 .
$$

Then, considering the impulse function defined in Section 3.2.1, this work proposes that pressure change at the wellbore in single-layer reservoirs with horizontal wells may be evaluated as:

$$
\Delta p\left(y, r=r_{w}, t\right)=q \int_{0}^{t} \Delta p_{y}(y, \tau) \mathcal{L}^{-1}\left\{\bar{G}_{w}\right\}(\tau) d \tau,
$$

where $\bar{G}_{w}$ is the impulse function defined in Eq. (3-19) and $\Delta p_{y}(y, \tau)$ stands for the impulse function in $y$-direction, as defined in Eq. (A-3). The images method can be applied to depict the reservoir vertical boundaries [33, 62].

Eq. (3-31) consists of an unorthodox application of Newman's [54, 55] product, since it is derived from a non-homogeneous reservoir model, as shown in Figs. 3.7 and 3.8. Appendix B offers a sketch proof for why Eq. (3-31) can describe the pressure change considering the reservoir model displayed in Figs. 3.7 and 3.8. A discussion regarding the accuracy of Eq. (3-31) is made in Section 3.3. A more solid proof for why Eq. (3-31) would be an interesting topic for further research but is out of the scope of this work.

\section{3 \\ Results and Discussion}

A comparison was made between a commercial flow simulator [45] and the formulations proposed in Section 3.2 in order to evaluate its accuracy on a set of synthetic cases. Additionally, the existing solutions for injectivity tests in multilayer reservoirs with vertical wells $[16,48]$ and single-layer reservoirs with horizontal wells [10] were also compared to the models presented in Section 3.2 .

In all cases, a piston-like fluid displacement was considered. The injection period was set as $96 \mathrm{~h}$. This time range is expected to be long enough for a radial flow regime to be identified in all cases. Relative permeability data may be seen in Fig. 3.9. These relative permeability set corresponds to the endpoints of the relative permeability curves displayed in Fig. 2.3. However, since the formulation presented in Section 3.2 assumes a piston-like fluid displacement, only the relative permeability endpoints are considered in this Chapter.

Besides, wellbore storage effects were neglected, to assure that some characteristic features of two-phase flow behavior may be observed (such as the derivative signature that occurs in reservoirs with formation damage $[10,14]$ ). 


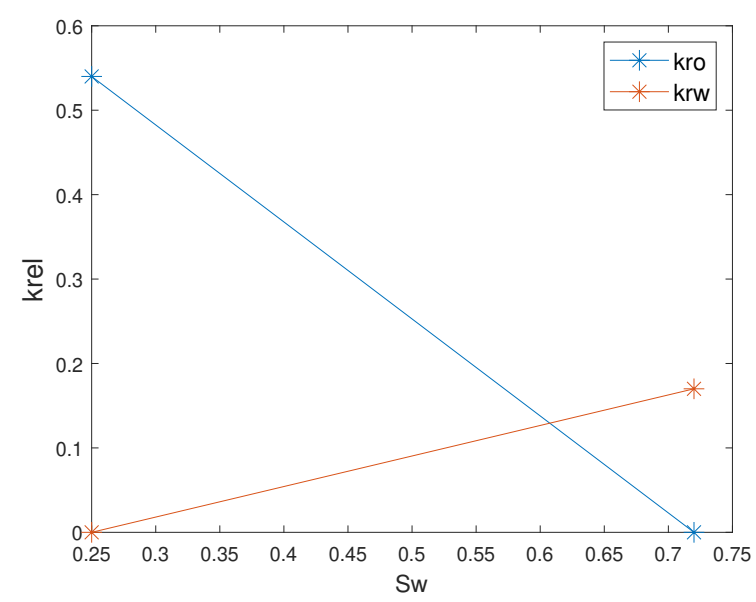

Figure 3.9: Relative permeability data

\subsection{1}

\section{Results for Multilayer Reservoirs with Vertical Wells}

The formulation proposed in Section 3.2.2 was implemented and compared to a commercial flow simulator [45]. A cylindrical reservoir grid was employed, with 70 blocks in the radial direction and 1 block in the angular direction. In the vertical direction, each grid block represented a distinct layer. The reservoir outer radius was set as $4 \mathrm{~km}$. The innermost grid block is 0.1 diameter wide and blocks get coarser as the distance to the wellbore increases.

The solutions developed by Barreto et al. [16] and Mastbaum et al. [48] for injectivity tests in multilayer reservoirs were also implemented and compared to the suggested model. Table 3.1 displays the reservoir properties for the multilayer cases. In all cases, a constant injection flow-rate of $500 \mathrm{~m}^{3} / \mathrm{d}$ was considered.

Fig. 3.10 displays the loglog graphs of pressure and pressure derivative for Case A. Pressure data are represented in black and derivative curves are shown in blue. The formulation suggested in this work is indicated by circles. Triangles

\begin{tabular}{|c|c|c|c|c|c|c|c|}
\hline Case & $\begin{array}{c}\mu_{o} \\
(\mathrm{cP})\end{array}$ & $\begin{array}{c}k \\
(\mathrm{mD})\end{array}$ & $\begin{array}{c}h \\
(\mathrm{~m})\end{array}$ & $\phi$ & $\begin{array}{c}k_{s} \\
(\mathrm{mD})\end{array}$ & $\begin{array}{c}r_{s} \\
(\mathrm{~m})\end{array}$ & $S$ \\
\hline \multirow{3}{*}{ A } & \multirow{3}{*}{4.8} & 500 & 15 & 0.11 & \multirow{3}{*}{200 (all) } & \multirow{3}{*}{0.4 (all) } & 2.1 \\
\hline & & 600 & 20 & 0.25 & & & 2.8 \\
\hline & & 700 & 25 & 0.33 & & & 3.5 \\
\hline \multirow{3}{*}{ B } & \multirow{3}{*}{1.1} & 500 & 15 & 0.11 & \multirow{3}{*}{200 (all) } & \multirow{3}{*}{0.4 (all) } & 2.1 \\
\hline & & 600 & 20 & 0.25 & & & 2.8 \\
\hline & & 700 & 25 & 0.33 & & & 3.5 \\
\hline \multirow{2}{*}{$\mathrm{C}$} & \multirow{2}{*}{4.8} & 500 & 20 & 0.11 & \multirow{2}{*}{200 (all) } & \multirow{2}{*}{0.4 (all) } & 2.1 \\
\hline & & 700 & 15 & 0.25 & & & 3.5 \\
\hline
\end{tabular}

Table 3.1: Reservoir properties for the multilayer cases 


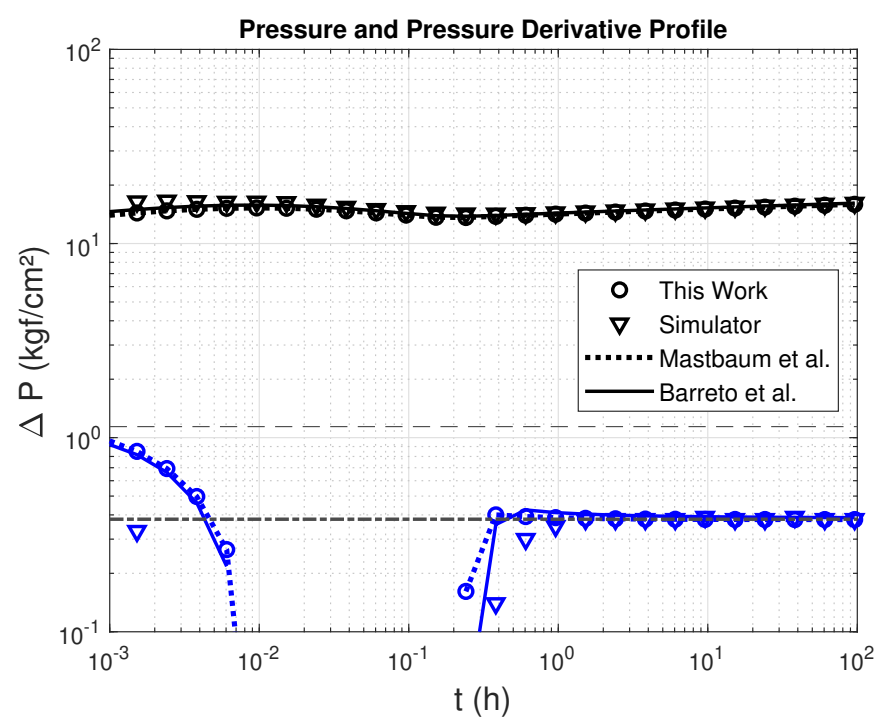

Figure 3.10: Pressure and pressure derivative curves for Case A

denote the numerical simulator. The dashed lines stand for the model proposed by Mastbaum et al. [48] and the solid lines represent the solution developed by Barreto et al. [16]. The horizontal dashed line indicate the theoretical derivative level associated with oil properties, while the horizontal line in dash-dot pattern stands for the water properties theoretical derivative level.

A great agreement between all methods is observed. The sharp derivative drop that occurs at early times reflects the existence of skin effects $[10,14,32]$. Pressure derivative considering the numerical simulator data shows some divergence from the analytical solutions during this period. This may be related to the numerical grid discretization. Besides, the piston-like fluid displacement

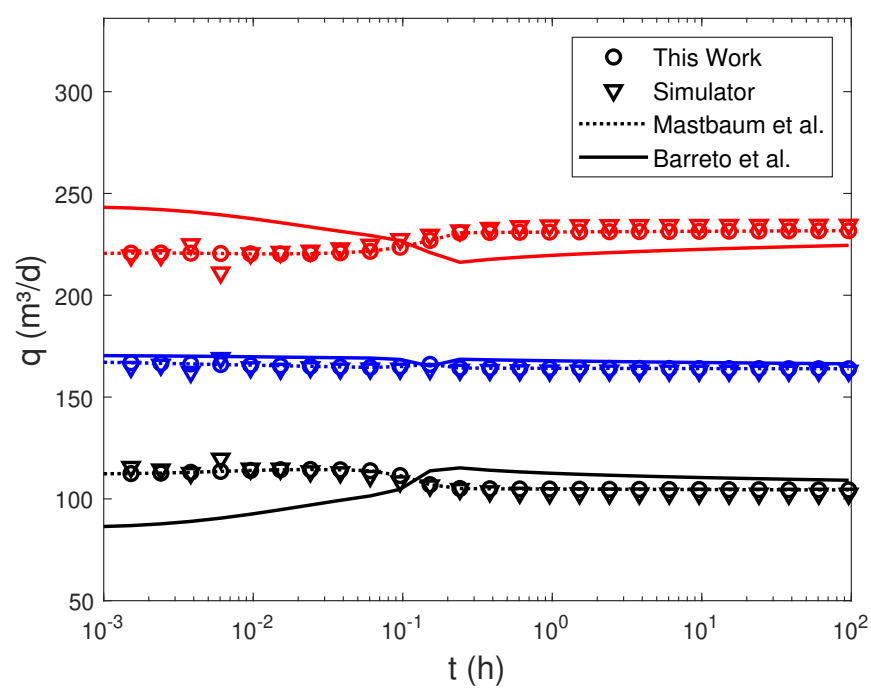

Figure 3.11: Layer flow-rate profiles for Case A (layer 1 in black; layer 2 in blue; layer 3 in red) 
is more accurately depicted by the analytical models than the flow simulator. Still, after the damaged region is swept by water, all derivative curves converge.

It is also important to highlight that all methods attained the theoretical derivative level associated to the water properties. This indicates that the proposed formulation may be applied to estimate the reservoir equivalent permeability, based on the constant derivative level $[9,12]$. This procedure to estimate the reservoir equivalent permeability was also explained in Section 2.4. Fig. 3.10 also shows that the derivative curves attain values close to the oil derivative level at early-times but quickly detaches from this theoretical level, as the derivative drop starts.

Fig. 3.11 presents the layer flow-rate profiles for each methods, in semilog scale. The black curves denote layer 1, while blue curves stand for layer 2 and red curves represent layer 3. Fig. 3.11 evidences that layer flow-rates at the wellbore change in time. Hence, as mentioned in Section 3.1, it is unfeasible to assure that flow-rate within the swept zone remains constant in multilayer reservoirs. Layer 1 presents the lowest flow capacity (that is, the permeabilitythickness product), while layer 3 presents the highest flow capacity. Thereby, flow-rate in layer 1 is lower than in layer 2, which is lower than in layer 3 .

As disclosed in Fig. 3.11, the proposed formulation exhibits a close convergence to the simulated data and the analytical model from Mastbaum et al. [48]. However, flow-rate profiles computed using the analytical solution developed by Barreto et al. [16] diverged from the other methods in layers 1 and 3. This result is particularly interesting, since accurate knowledge over layer flow-rate data is crucial for parameter estimation techniques [5, 13, 56, 57].

Nonetheless, it is interesting to notice that the formulation proposed by Barreto et al. was able to provide pressure and pressure derivative curves that accurately match the other methods, despite the divergence observed in the layer flow-rate profiles.

Pressure and pressure derivative curves for case B may be seen in Fig. 3.12. Case B presents the same reservoir properties as Case A, except for oil viscosity. Therefore, the theoretical oil derivative level is now lower than the water derivative level. The hump in pressure derivative curves that occurs between $t \approx 0.01 \mathrm{~h}$ and $t \approx 0.2 \mathrm{~h}$ reflects the existence of skin effects in reservoirs where flow is favorable to oil [14, 32]. After this derivative hump, flood front propagates throughout the undamaged zone.

Similarly to Case A, all methods presented a close agreement. The three analytical formulations converged during the entire test, while the numerical data presented some slight divergences at early times, when the flood front is still within the damaged zone. Despite that, the overall agreement is quite 


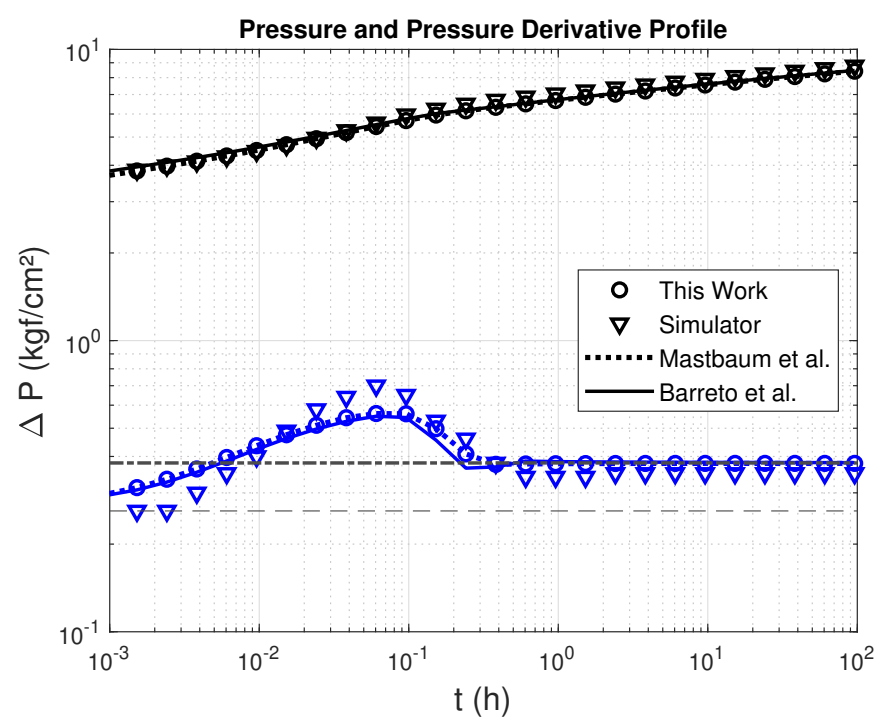

Figure 3.12: Pressure and pressure derivative curves for Case B

good and all methods converged towards the theoretical water derivative level, indicating that late-time data can be used to determine the reservoir equivalent permeability.

Layer flow-rate for Case B are reported in Fig. 3.13. The formulation suggested in Section 3.2 exhibits an excellent agreement with the solution developed by Mastbaum et al. [48]. Flow-rates evaluated using these analytical models show a similar behavior to the numerical simulator flow-rate curves. Yet, in layers 1 and 3, the match between analytical and simulated data is not as close as observed in Case A.

On the other hand, flow-rate profiles determined by the solution proposed

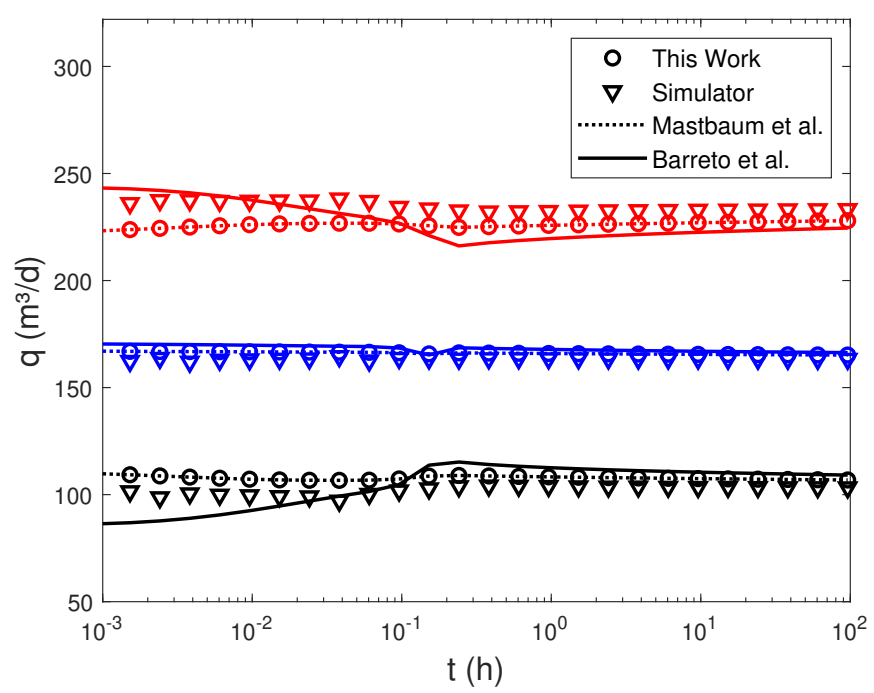

Figure 3.13: Layer flow-rate profiles for Case B (layer 1 in black; layer 2 in blue; layer 3 in red) 


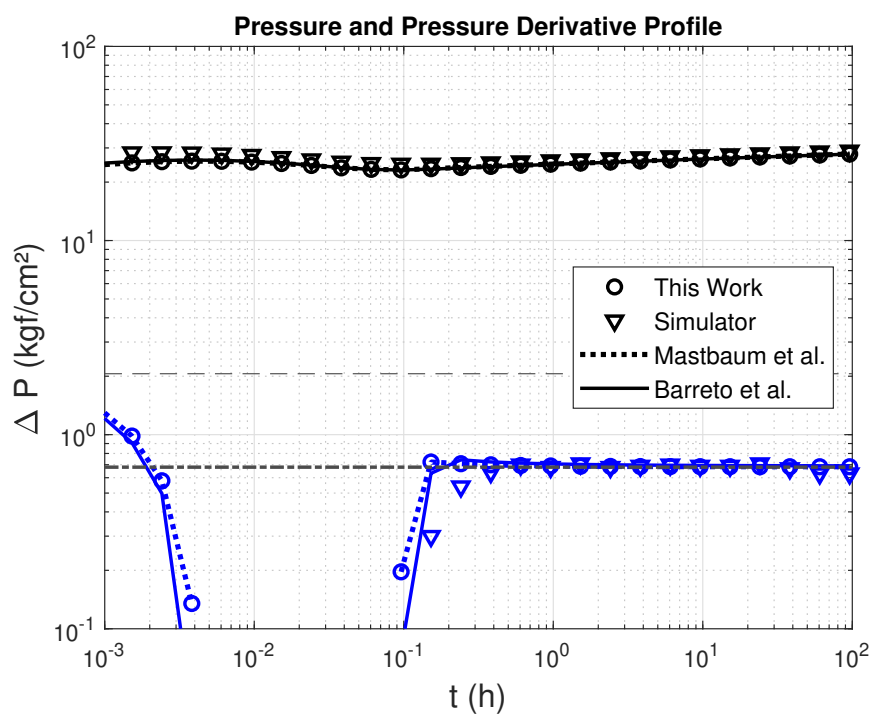

Figure 3.14: Pressure and pressure derivative curves for case $\mathrm{C}$

by Barreto et al. [16] presented noticeable differences compared to the other methods. Figs. 3.12 and 3.13 show that the solution from Barreto et al. [16] provided a good match for pressure data but yielded layer flow-rate profiles that diverged from the other models, as occurred in Case A.

Pressure and derivative data for Case C may be observed in Fig. 3.14. The overall behavior is similar to Case A: pressure change presented a close agreement for all methods, as well as the derivative curves. The blunt derivative drop at early-times indicates the existence of formation damage. At late-times, the theoretical derivative level associated with water properties is attained by all methods.

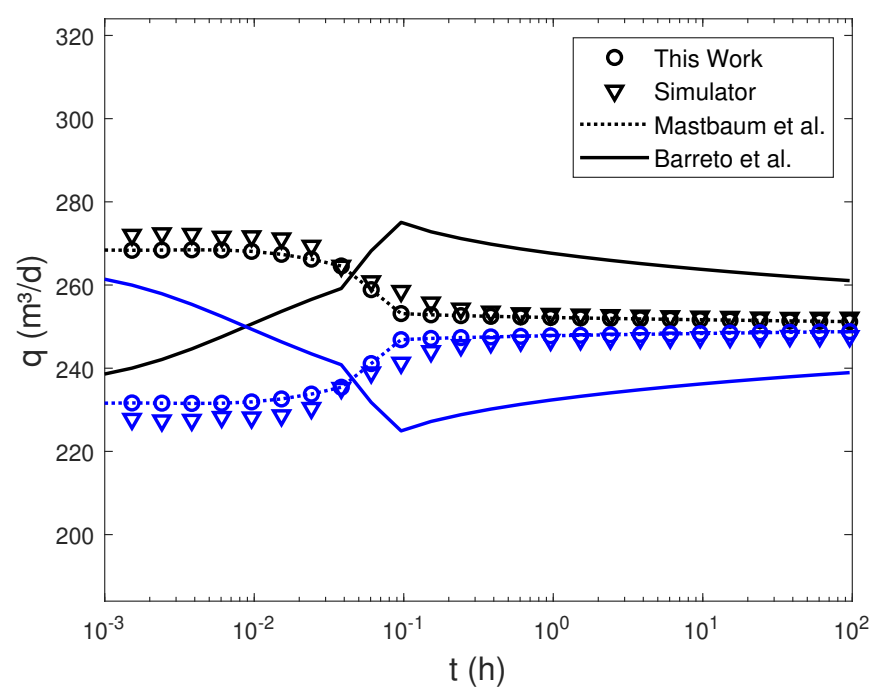

Figure 3.15: Layer flow-rate profiles for case C (layer 1 in black; layer 2 in blue) 
In Case C, layer flow-rate curves, portrayed in Fig. 3.15, provide a more interesting analysis than pressure and derivative data. Fig. 3.15 evidence that both the formulation proposed in Section 3.2 and the solution presented by Mastbaum et al. [48] exhibited a great agreement with the numerical data. As mentioned earlier, accurate evaluation of flow-rates in each layer is helpful to some interpretation techniques that aim to estimate individual layer properties $[5,13,56,57]$.

Similar to the previous cases, flow-rates computed using the solution from Barreto et al. [16] diverged from the other analytical models and from the numerical simulator. Moreover, the solution from Barreto et al. [16] presented the most significant variations in layer flow-rates, even though this is the only analytical model that requires flow-rate to be constant within the swept region. Despite that, it is interesting to notice the close agreement presented by the pressure and derivative curves in Fig. 3.14.

Pressure and layer flow-rate profiles displayed in Figs. 3.10 to 3.15 were employed to estimate layer permeabilities using the Delta Transient Method $[56,57]$. This procedure demands pressure and flow-rate data from only two distinct time steps, which is a significant operational advantage [56, 57].

In this Section, the late-time logarithmic approximation based on water properties was employed to evaluate layer permeabilities. For all methods, pressure and flow-rate data at $t=6.06 \mathrm{~h}$ and $t=60.6 \mathrm{~h}$ were used to evaluate layer permeabilities. Figs. 3.10, 3.12 and 3.14 evidence that, for all cases, a radial flow regime occurs during this time range, since derivative remains constant.

Skin factors were not estimated in this Section, since pressure change in reservoirs under water injection exhibit not only a mechanical skin due to formation damage but also an apparent skin derived from the mobility contrast within the swept zone $[11,32]$. For this reason, the determination of layer mechanical skins is out of the scope of this work.

Table 3.2 displays the estimated layer permeabilities considering each approach employed to compute the pressure change. A great accuracy may be observed in all cases. Moreover, the formulation presented in this thesis yielded values very close to the estimates obtained from the flow simulator and the model proposed by Mastbaum et al. [48]. The solution developed by Barreto et al. [16], in its turn, showed some differences compared to the other models (particularly in case B), despite presenting a decent accuracy.

These divergences are likely related to the mismatch between layer flowrate data. As displayed in Figs. 3.11, flow-rates evaluated using the solution developed by Barreto et al. [16] diverged from the other approaches, which 


\begin{tabular}{|cc|cc|cc|cc|cc|}
\cline { 3 - 10 } \multicolumn{1}{c|}{} & \multicolumn{2}{c|}{ This Work } & \multicolumn{2}{c|}{ Num. Sim. } & \multicolumn{2}{c|}{ Mastbaum et al. } & \multicolumn{2}{c|}{ Barreto et al. } \\
\hline \multirow{2}{*}{ Case } & True & Est. & Error & Est. & Error & Est. & Error & Est. & Error \\
\hline \multirow{2}{*}{$\mathrm{A}$} & 500 & 498 & -0.3 & 493 & -1.4 & 498 & -0.3 & 429 & -14.2 \\
& 600 & 599 & -0.2 & 589 & -1.8 & 599 & -0.2 & 560 & -6.7 \\
& 700 & 700 & 0.0 & 691 & -1.3 & 700 & 0.0 & 767 & 9.6 \\
\hline \multirow{3}{*}{$\mathrm{B}$} & 500 & 501 & 0.1 & 500 & 0.1 & 501 & 0.1 & 481 & -3.7 \\
& 600 & 600 & 0.1 & 599 & -0.2 & 600 & 0.1 & 590 & -1.6 \\
& 700 & 700 & -0.1 & 700 & 0.0 & 700 & -0.1 & 716 & 2.2 \\
\hline \multirow{2}{*}{$\mathrm{C}$} & 500 & 499 & 0.2 & 497 & -0.7 & 499 & -0.2 & 448 & -10.4 \\
& 700 & 701 & 0.1 & 683 & -2.4 & 701 & 0.1 & 804 & 14.8 \\
\hline
\end{tabular}

Table 3.2: Estimated layer permeabilities (values in $\mathrm{mD}$, errors in percentage)

yielded similar flow-rate profiles. These results combined with the higher errors in layer permeabilities observed in Table 3.2 suggest that layer flowrate profiles computed using their model are less accurate than the flow-rate curves obtained from the other methods.

\subsection{2}

\section{Results for Single-Layer Reservoirs with Horizontal Wells}

A computational implementation of the analytical model presented in Section 3.2.3 was developed and compared to the solution proposed by Peres and Reynolds [10] for injectivity tests in single-layer reservoirs with horizontal wells. Results were also compared to the commercial flow simulator [45].

A cartesian grid composed of $61 \times 61$ x 9 blocks was employed. Again, blocks closer to the wellbore were more refined than blocks further from it. Reservoir grid was 6,000 m wide ( $x$-direction) and 6,300 $\mathrm{m}$ long ( $y$-direction). The wellbore was built parallel to the $y$-direction. Length ( $y$-direction) of each block containing the wellbore was set as $100 \mathrm{~m}$, while width ( $x$-direction) and height ( $z$-direction) were set as $0.89 \mathrm{~m}$. Therefore, the skin zone radius was set as $0.5 \mathrm{~m}$ in the analytical models, so that the damaged region presents the same cross-sectional area that the numerical simulation grid, as explained in Section 2.4. A hybrid refinement of 4 (radial direction) by 4 (angular direction) by 4 (well axis direction) was employed at all grid blocks containing the wellbore.

The numerical simulator considers an infinite conductivity wellbore, while both analytical solutions assume uniform influx. As mentioned in Chapter 2 , either the average pressure technique $[22,23,51]$ or the equivalent pressure point $[18,21,33,35]$ may be applied to obtain the pressure change at an infinite conductivity wellbore using a formulation that assumes uniform influx. While the average pressure technique may be more accurate, its implementation is also more complex. For this reason, in this Chapter, the equivalent pressure point was employed to obtain the pressure change corresponding to 


\begin{tabular}{|ccccccccc|}
\hline Case & $\begin{array}{c}\mu_{o} \\
(\mathrm{cP})\end{array}$ & $\begin{array}{c}k \\
(\mathrm{mD})\end{array}$ & $\begin{array}{c}h \\
(\mathrm{~m})\end{array}$ & $\begin{array}{c}L \\
(\mathrm{~m})\end{array}$ & $\phi$ & $\begin{array}{c}k_{s} \\
(\mathrm{mD})\end{array}$ & $\begin{array}{c}r_{s} \\
(\mathrm{~m})\end{array}$ & $S$ \\
\hline $\mathrm{D}$ & 4.8 & 2000 & 25 & 700 & 0.25 & 500 & 0.5 & 4.8 \\
\hline $\mathrm{E}$ & 1.1 & 2000 & 25 & 700 & 0.25 & 500 & 0.5 & 4.8 \\
\hline
\end{tabular}

Table 3.3: Reservoir properties for the cases with horizontal well

an infinite conductivity wellbore. Thus, pressure change in the analytical formulations was evaluated at $x=z=r_{w}$ and $y=0.732$ times the wellbore length, as suggested by Ozkan et al. [18].

Layer properties for the tested cases may be found in Table 3.3. Injection flow-rate was defined as $5,000 \mathrm{~m}^{3} / \mathrm{d}$ for Cases D and E. Reservoir properties are the same in both cases, except for the oil viscosity. The horizontal well was assumed to be at the center of the layer; that is, no off-centering was considered. Waterfront radius at the end of the $96 \mathrm{~h}$ injection period, determined by Eq. (2-3), was equal to $8.8 \mathrm{~m}$. Thus, the flood front has not yet reached the reservoir vertical boundaries and the formulation developed in Section 3.2.3 is applicable.

Fig. 3.16 displays the pressure and pressure derivative data for Case D. Black curves denote the pressure data while the blue curves correspond to the derivative profile. The model proposed in Section 3.2.3 is indicated by circles. Triangles represent the numerical simulator and the solid lines correspond to the solution presented by Peres and Reynolds [10]. The horizontal dashed line indicates the theoretical pressure derivative profile for early-time radial flow, computed from Eq. (2-29). At late-times, the flood front is still propagating in the vertical plane, while pressure diffusion is occurring in the horizontal

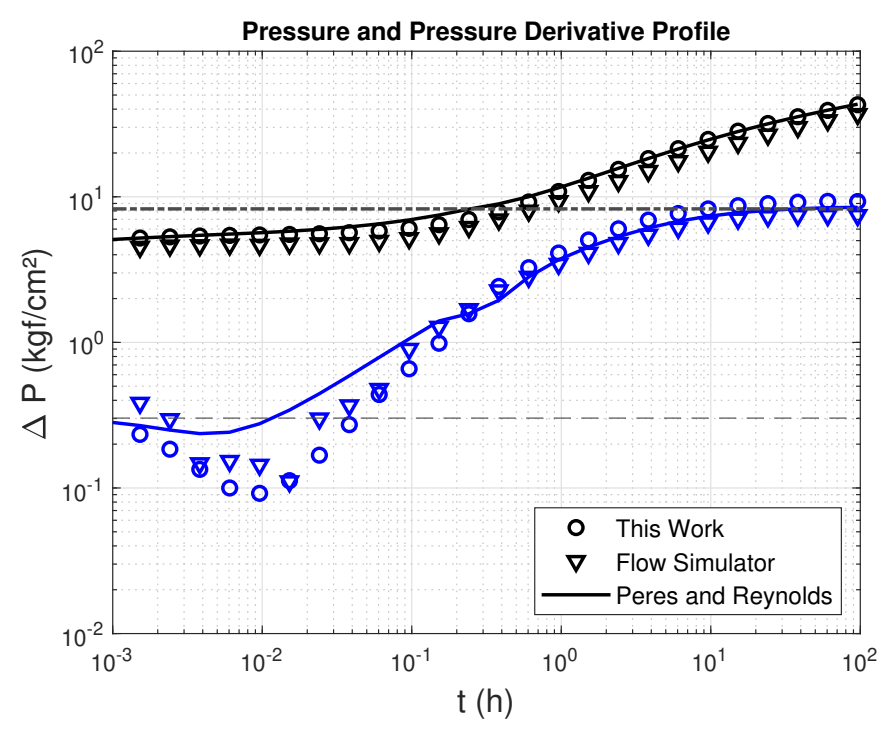

Figure 3.16: Pressure and pressure derivative curves for case D 


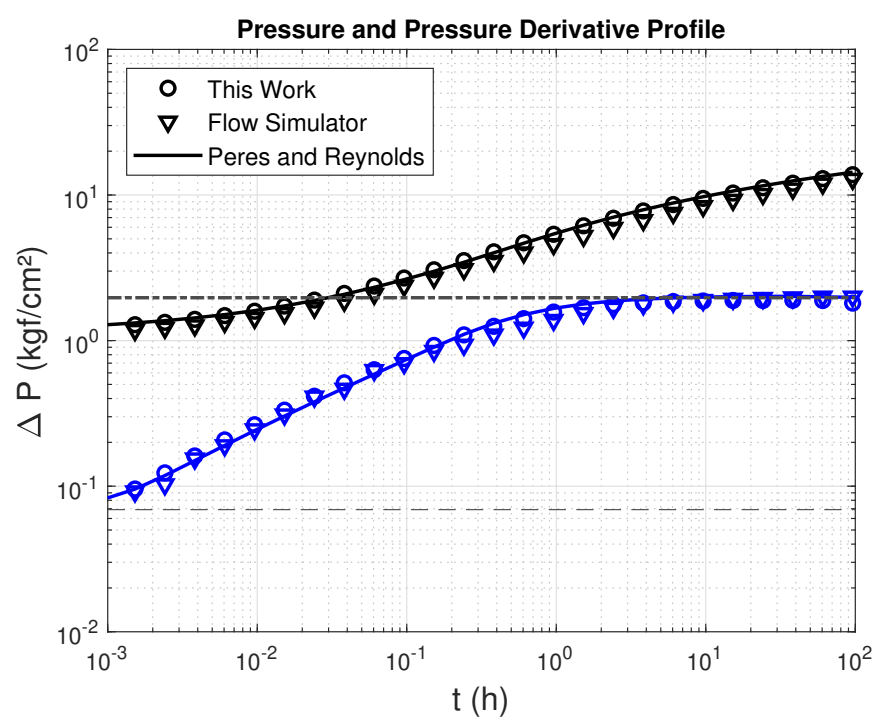

Figure 3.17: Pressure and pressure derivative curves for case E

direction. Therefore, a "second radial - first radial" occurs at the end of the test. The dash-dotted horizontal line stands for the theoretical level for this flow regime, determined from Eq. (2-31).

A good agreement is observed between all curves. As in Section 3.3.1, the pressure derivative drop observed at early-times indicates the existence of formation damage. Pressure derivative for the numerical simulator presents some oscillations for short times $\left(t \leq 3 \cdot 10^{-2} \mathrm{~h}\right)$. These oscillations are possibly explained by the grid discretization and how the numerical simulator accuracy to represent a piston-like flow. Despite that, numerical data match well the analytical models.

It is worthy to mention that, interestingly enough, the proposed formulation based on an unconventional application of Newman's [54, 55] product presents a closer match to the simulator derivative curve than the solution developed by Peres and Reynolds [10] at early-times. Furthermore, all methods attain the late-time theoretical derivative level.

The results for Case E may be seen in Fig. 3.17. All methods exhibit a great match not only for pressure but also for derivative data. In Case E, the derivative curves quickly detach from the early-time theoretical level. Still, the derivative signature that indicates a late-time radial flow is clearly observed. As explained in Section 2.3.4, this constant derivative level is useful to determine the reservoir permeability $[6,33]$.

Since Case E presents a mobility ratio lower than unity, no derivative drop is observed. One could expect a derivative hump instead, similar to cases $\mathrm{B}$ and $\mathrm{C}$. However, this hump may be masked by the derivative rise due to the linear flow regime. Yet, it is interesting to notice once again the close agreement 
presented by the proposed solution, considering the application of Newman's $[54,55]$ product for a non-homogeneous reservoir model.

\section{4}

\section{Summary and Conclusions}

This work proposes an impulse function for two-phase flow in laterally infinite reservoirs. The proposed formulation applies a radially composite reservoir approach to represent the flooded zone and the uninvaded region. The main findings and conclusions of this work are listed below:

- The solution developed in this work does not require flow-rate within the swept area to remain constant, unlike previous models for injectivity tests based on the Thompson and Reynolds' [15] steady-state theory.

- An approximate solution for injectivity tests in single-layer reservoirs with horizontal wells is also obtained, performing an unconventional application of Newman's product $[54,55]$, considering a non-homogeneous domain.

- Pressure data evaluated using the formulation proposed in this work presented a close agreement when compared to a commercial flow simulator and to previously existing analytical solutions $[10,16,48]$.

- Layer flow-rate profiles evidenced that flow-rates at the wellbore indeed may change in time, endorsing that, in a rigorous sense, the Thompson and Reynolds' [15] theory is not applicable for multilayer reservoirs.

- Results from Section 3.3.2 verify that the unorthodox application of Newman's product $[54,55]$ suggested in Section 3.2.3 was able to provide a formulation for injectivity tests in single-layer reservoirs with horizontal wells that matches well the results from a commercial flow simulator and also the solution developed by Peres and Reynolds' [10]. A solid proof to justify the application of Newman's [54] product using a radially heterogeneous reservoir model was out of the scope of this work, and remains as suggestion for further research.

- The proposed model is based on some simplifying hypothesis. Assuming an infinite acting reservoir and piston-like fluid displacement are likely the most unrealistic assumptions. Moreover, the results in Section 2.4 assume that the injection flow-rate at the wellbore remains constant. Overcoming these limitations would also be interesting topics for future research. 


\section{Summary and Suggestions for Future Work}

This work has two main goals: the first one is to reach an analytical model for injection/falloff tests in multilayer stratified reservoirs with multilateral wells. The second is to evaluate pressure change in reservoirs with vertical wells using impulse functions.

Chapter 2 outlines how the previously existing solutions for injectivity tests in single-layer reservoirs $[10,11]$ can be extended to multilayer stratified formations with multilateral horizontal wells.

The formulations introduced in Chapter 2 are analogous to the equations that describe pressure behavior in multilayer formations with vertical wells $[12,16]$. Besides, theoretical levels for pressure derivative were also presented, according to the several flow regimes that may occur in reservoirs with horizontal wells. Chapter 2 also shows that the suggested model presented a good match when compared to a commercial flow-simulator and that the reservoir equivalent permeability may be estimated from the pressure derivative profile.

Chapter 3 introduces the developed analytical model based on Green's functions for vertical wells. Additionally, an unorthodox application of Newman's $[54,55]$ product is proposed to obtain the pressure change in singlelayer reservoirs with horizontal wells, considering a radially composite reservoir model.

Results exhibited in Chapter 3 validate the applicability of the proposed formulation using impulse functions. Chapter 3 also illustrates that layer flow-rates at the wellbore may change in time. This outcome evidences that, for multilayer reservoirs, flow-rate within the flooded zone is not necessarily constant. Thus, the analytical models developed by Barreto et al. [16], Bela et al. [12] and in Chapter 2 present an inconsistency, as they are based on the Thompson and Reynolds' [15] steady-state theory. Moreover, the Delta Transient Method $[56,57]$ was successfully applied to estimate individual layer permeabilities.

The work presented in this thesis also may used as basis for further research. Some suggestions for future works are given below.

- Apply semilog pressure interpretation techniques, such as Rate- 
Normalized Pressure Analysis [5, 44] or the Delta Transient Method $[56,57]$, to estimate individual layer properties based on the pressure transient data obtained through the formulation proposed in this work for injectivity tests in multilayer reservoirs with multilateral horizontal wells;

- Alternatively, the developed formulations may also be coupled to history matching procedures in order to estimate reservoir parameters.

- Extend the formulation presented in Chapter 3 for injectivity tests in single-layer reservoirs so that it can be applied to multilayer reservoirs with multilateral horizontal wells. The model presented in Chapter 2 may be used as benchmark in this case;

- Develop the solution for the falloff period using impulse functions based on the model proposed in Chapter 3;

- Adapt the formulation presented in Chapter 3 to handle the flood front more accurately than assuming piston-like fluid displacement;

- Provide a more rigorous proof for why the application of Newman's $[54,55]$ product suggested in Section 3.2.3 works and verify if its possible application for other non-homogeneous domains. 


\section{Bibliography}

[1] EARlOugheR JR., R. C.. Advances in Well Test Analysis. Número 5 em Monograph Series. SPE, Richardson, Texas, USA, 1977.

[2] AHMED, T.. Reservoir Engineering Handbook. Elsevier, Oxford, UK, 2010.

[3] GAO, C.-T.. Determination of parameters for individual layers in multilayer reservoirs by transient well tests. SPE Formation Evaluation, March:43-65, 1987.

[4] GOODE, P. A.; ThAMBYnayAGAM, R. K. M.. Pressure drawdown and buildup analysis of horizontal wells in anisotropic media. SPE Formation Evaluation, December:683-697, 1987.

[5] EHLIG-ECONOMIDES, C. A.; JOSEPH, J.. A new test for determination of individual layer properties in a multilayered reservoir. SPE Evaluation Formation, September:261-283, 1987.

[6] ODEH, A. S.; BABU, D. K.. Transient flow behavior of horizontal wells, pressure drawdown and buildup analysis. SPE Formation Evaluation, March:7-15, 1990.

[7] BORELLO, E. S.; FOKKER, P. A.; VIBERTI, D.; VERGA, F.; HOFMANN, H.; MEIER, P.; MIN, K.-B.; YOON, K. ; ZIMMERMANN, G.. Harmonic pulse testing for well monitoring: Application to a fractured geothermal reservoir. Water Resources Research, 55:4727-4744, 2019.

[8] BOnAFÉ, M. F.; BRAgA, A. ; BARReto JR., A. B.. Approximate solution for pressure during a multiple rate injectivity test. Journal of Petroleum Exploration and Production Technology, 10:2373-2386, 2020.

[9] BANERJEE, R.; THOMPSON, L. G. ; REYNOLDS, A. C.. Injection/falloff testing in heterogeneous reservoirs. SPE Reservoir Evaluation \& Engineering, December:519-527, 1998.

[10] PERES, A. M. M.; REYNOLDS, A. C.. Theory and analysis of injectivity tests on horizontal wells. SPE Journal, June:147-159, 2003. 
[11] Peres, A. M. M.; BOUgharA, A. A. ; ReYNoldS, A. C.. Rate superposition for generating pressure falloff solutions. SPE Journal, September:364-374, 2006.

[12] BELA, R. V.; PESCO, S. ; BARRETO JR., A. B.. Modeling falloff tests in multilayer reservoirs. Journal of Petroleum Science and Engineering, 174:161-168, 2019.

[13] COUTINHO, E. J. R.; EMERICK, A. A.; LI, G. ; REYNOLDS, A. C.. Conditioning multilayered geologic models to well-test and production-logging data using the ensemble kalman filter. paper presented at SPE Annual Technical Conference and Exhibition, September:119, 2010.

[14] SILVA, T. D. M. D.; BELA, R. V.; PESCO, S. ; BARRETO JR., A. B.. Esmda applied to estimate skin zone properties from injectivity tests data in multilayer reservoirs. Computers \& Geosciences, 146:1$18,2021$.

[15] THOMPSON, L. G.; REYNOLDS, A. C.. Well testing for radially heterogeneous reservoirs under single and multiphase flow conditions. SPE Evaluation Formation, March:57-64, 1997.

[16] BARRETO JR., A. B.; PERES, A. M. M. ; PIRES, A. P.. Water injectivity tests on multilayered oil reservoirs. Paper presented at the Brasil Offshore Conference and Exhibition, June:1-11, 2011.

[17] BOUGHARA, A. A.; REYNOLDS, A. C.. Analysis of injection/falloff data from horizontal wells. SPE Journal, December:721-736, 2009.

[18] OZKAN, E.; RAGHAVAN, R. ; JOSHI, S. D.. Horizontal well pressure analysis. SPE Formation Evaluation, December:567-575, 1989.

[19] ZHAN, H.; ZLOTNIK, V. A.. Groundwater flow to a horizontal or slanted well in an unconfined aquifer. Water Resources Research, 38:1-11, 2002.

[20] LefKoVITS, H. C.; HAZEBROEK, P.; ALLEN, E. E. ; MATTHEWS, C. S.. A study of the behavior of bounded reservoirs composed of stratified layers. SPE Journal, March:43-58, 1961.

[21] DaViau, F.; MOURONVAI, G.; BOURdAROT, G. ; CURUTCHeT, P.. Pressure analysis for horizontal wells. SPE Formation Evaluation, December:716-724, 1988. 
[22] KUCHUK, F. J.; GOODE, P. A.; WILSKINSON, D. J. ; THAMBYNAYAGAM, R. K. M.. Pressure-transient behavior for horizontal wells with and without gas cap or aquifer. SPE Formation Evaluation, March:8694, 1991.

[23] KUCHUK, F. J.; HABASHY, T.. Pressure behavior of horizontal wells in multilayer reservoirs with crossflow. SPE Formation Evaluation, March:55-64, 1996.

[24] NIE, R.-S.; GUO, J.-C.; JIA, Y.-L.; ZHU, S.-Q.; RAO, Z. ; ZHANG, C.-G.. New modelling of transient well test and rate decline analysis for a horizontal well in a multiple-zone reservoir. Jornal of Geophysiscs and Engineering, 8:464-476, 2011.

[25] CHEN, Z.; LIAO, X.; ZHAO, X.; DOU, X.; ZHU, L. ; SANBO, L.. A finiteconductivity horizontal well model for pressure-transient analysis in multiple-fractured horizontal wells. SPE Journal, 22:11121122, 2017.

[26] WANG, Q.; ZHAN, H.. Intrawellbore kinematic and frictional losses in a horizontal well in a bounded confined aquifer. Water Resources Research, 53:127-141, 2017.

[27] YILDIZ, T.. Multilateral pressure-transient response. SPE Journal, March:5-12, 2003.

[28] YILDIZ, T.. Multilateral horizontal well productivity. paper presented at the SPE Europec/EAGE Annual Conference, June:1-8, 2005.

[29] VO, D. T.; MADDEN, M. V.. Performance evaluation of trilaterals: Field examples. SPE Reservoir Engineering, February:22-28, 1995.

[30] LARSEN, L.. Pressure-transient behavior of multibranched wells in layered reservoirs. SPE Reservoir Evaluation \& Engineering, February:68-73, 2000.

[31] PAN, Y.; KAMAL, M. M. ; KIKANI, J.. Field applications of a semianalytical model of multilateral wells in multilayer reservoirs. SPE Reservoir Evaluation \& Engineering, December:861-872, 2010.

[32] BELA, R. V.; PESCO, S. ; BARRETO JR., A. B.. Determining skin zone properties from injectivity tests in single and multilayer reservoirs. Journal of Petroleum Exploration and Production Technology, 10:1459-1471, 2020. 
[33] ROSA, A. J.; CARVALHO, R. D. S.. A mathematical model for pressure evaluation in an infinite-conductivity horizontal well. SPE Formation Evaluation, December:559-566, 1989.

[34] ABBASZADEH, M.; HEGEMAN, P. S.. Pressure-transient analysis for a slanted well in a reservoir with vertical pressure support. SPE Formation Evaluation, September:277-284, 1990.

[35] JELMERT, T. A.; THOMPSON, L. G.. Horizontal wells - a test on infinite conductivity solutions. Paper presented at SPE International Arctic Technology Conference, May:1-20, 1991.

[36] SPIVEY, J. P.; LEE, W. J.. Estimating the presssure-transient response for a horizontal or a hydraulically fractured well at an arbitrary orientation in an anisotropic reservoir. SPE Reservoir Evaluation \& Engineering, October:462-469, 1999.

[37] SPATH, J. B.; OZKAN, E. ; RAGHAVAN, R.. An efficient algorithm for computation of well responses in commingled reservoirs. SPE Formation Evaluation, June:115-121, 1994.

[38] POPA, C. G.; TURTA, A. T.. Waterflooding by horizontal injectors and producers. Paper presented at SPE International Thermal Operations and Heavy Oil Symposium and International Horizontal Well Technology Conference, November:1-13, 2002.

[39] ALGHARAIB, M.; GHARBI, R. ; MALALLAH, A.. Scaling immiscible displacement in porous media with horizontal wells. Transport in Porous Media, 65:89-105, 2006.

[40] BUCKLEY, S. E.; LEVERETT, M. C.. Mechanism of fluid displacement in sands. Transactions of the AIME, 146:107-116, 1942.

[41] DEPPE, J. C.. Injection rates - the effect of mobility ratio, area swept and pattern. SPE Journal, June:81-91, 1961.

[42] ABBASZADEH, M.; KAMAL, M.. Pressure-transient testing of waterinjection wells. SPE Reservoir Engineering, 4:115-124, February 1989.

[43] BRATVOLD, R. B.; HORNE, R. N.. Analysis of pressure-falloff tests following cold-water injection. SPE Formation Evaluation, September:293-302, 1990.

[44] RAGHAVAN, R.. Behavior of wells completed in multiple producing zones. SPE Evaluation Formation, June:219-230, 1989. 
[45] CMG. IMEX User Guide. Computer Modeling Group, Calgary, Alberta, Canada, 2010.

[46] NETO, J. L. F. B.. Testes de injetividade em reservatórios multicamadas: Solução aproximada para um esquema de múltiplas vazões (in portuguese). Ms thesis, Pontifícia Universidade Católica do Rio de Janeiro (PUC-Rio), 2020.

[47] NETO, J. L. F. B.; BELA, R. V.; PESCO, S. ; BARRETO JR., A. B.. Pressure behavior during injectivity tests - a composite reservoir approach. Paper presented at the SPE Latin American and Caribbean Petroleum Engineering Conference, July:1-18, 2020.

[48] MASTBAuM, A. L.; SOUZA, A. B.; NETO, J. L. F. B.; BELA, R. V. ; BARRETO JR., A. B.. Dual-phase flow in multilayered porous media - a radial composite laplace domain approximation. preprint submitted to Journal of Petroleum Exploration and Production Technology, uploaded at archive.org, p. 1-15, 2021.

[49] VIANA, I. V.. An analytical model for injectivity tests in multilayered reservoirs with formation crossflow. Ms thesis, Pontifícia Universidade Católica do Rio de Janeiro (PUC-Rio), 2021.

[50] GRINGARTEN, A. C.; RAMEY JR., H. J.. The use of source and green's functions in solving unsteady-flow problems in reservoirs. SPE Journal, October:285-296, 1973.

[51] CHEN, H.-Y.; RAGHAVAN, R. ; POSTON, S. W.. An application of the product-solution principle for instantaneous source and green's functions. SPE Formation Evaluation, June:161-168, 1991.

[52] KUCHUK, F. J.; WILKINSON, D. J.. Transient pressure behavior of commingled reservoirs. SPE Formation Evaluation, March:111-120, 1991.

[53] LU, J.; ZHOU, B.; RAHMAN, M. M. ; HE, X.. New solution to the pressure transient equation in a two-layer reservoir with crossflow. Journal of Computational and Applied Mathematics, 362:680693, 2019.

[54] NEWMAN, A. B.. Heating and cooling rectangular and cylindrical solids. Industrial and Engineering Chemistry, 28:545-548, 1936. 
[55] CARSLAW, H. S.; JAEGER, J. C.. Conduction of Heat in Solids. Número Section 1.15 em Second Edition. Oxford University Press, London, U.K., 1959.

[56] GALVÃO, M. S. C.; GUIMARÃES, C. S.. A new method for calculating individual layer permeability and skin in a multilayered reservoir using production logging data: The delta transient method. Paper presented at the SPE Latin America and Caribbean Mature Fields Symposium, March:1-30, 2017.

[57] GUIMARÃES, C. S.; GALVÃO, M. S. C.. Application of the delta transient method to multi-rate tests: A method for calculating individual layer permeability and skin in a multilayered reservoir using production logging data. Paper presented at the Offshore Technology Conference Brasil, October:1-22, 2017.

[58] LINO NETO, A. D. C.. Modelagem matemática de testes de pressão em reservatórios de gás com região de permeabilidade reduzida em torno dos poços (in portuguese). Ms thesis, Universidade Estadual do Norte Fluminense (UENF), 2019.

[59] PRATS, M.; MENESES-P., L. L.; SAMANEIGO-V., F. ; PACHECO-V., R.. Pressure buildup in a well produced at constant pressure. SPE Journal, 25:1578-1598, 2020.

[60] ABRAMOWITZ, M.; STEGUN, I. A.. Handbook of Mathematical Functions. Número 55 em Applied Mathematics Series. United States National Bureau of Standards, Washington, DC, 1964.

[62] CLONTS, M. D.; RAMEY JR., H. J.. Pressure-transient analysis for wells with horizontal drainholes. paper presented at SPE California Regional Meeting, April:215-230, 1986.

[63] EVERDINGEN, A. F. V.; HURST, W.. The application of the laplace transformation to flow problems in reservoirs. Petroleum Transactions, December:305-324, 1949. 
A

\section{Single-Phase Flow Through Multilateral Horizontal Wells}

This Appendix briefly presents the solution for single-phase flow in singlelayer reservoirs with horizontal wells, presented by [21] and [18]. Then, we extend this solution to multilayer systems, as proposed by [31]. The same simplifying hypotheses made in section 2.3 will be hereafter assumed.

\section{A.1}

\section{Single-Phase Flow in Single-Layer Reservoirs}

The solution for single-phase flow in single layer reservoirs is well known $[21,18]$. Considering that the wellbore heel is positioned at coordinates $\left(x_{1}, y_{1}, z_{1}\right)$ and applying Newmann's product, the pressure change $\Delta p$ at a given time $t$ may be decomposed as the product of three terms, such that each term is associated to flow in a given direction [33]:

$$
\Delta p(x, y, z, t)=\frac{q}{L \phi c_{t}} \int_{0}^{t} \Delta p_{x}(x, \tau) \Delta p_{y}(x, \tau) \Delta p_{z}(x, \tau) d \tau .
$$

In Eq. (A-1), $q$ stands for the flow-rate and the wellbore length is represented by $L$, while $\phi$ and $c_{t}$ denote the porosity and total compressibility, respectively. Eq. (A-1) represents the time integral of all instant pressure variations at a given point with coordinates $(x, y, z)$ due to the source point productions along the well. Instant pressure change in $x$-direction is given by $[21,18]$ :

$$
\Delta p_{x}(x, t)=\frac{1}{2 \sqrt{\pi \eta_{x} t}} \exp \left(-\frac{\left(x-x_{1}\right)^{2}}{4 \eta_{x} t}\right),
$$

where $\eta_{x}=k_{x} / \phi \mu c_{t}$ stands for the hydraulic diffusivity coefficient in $x$ direction. Analogous hydraulic diffusivity coefficients are also defined in $y$ and $z$-directions.

In $y$-direction, the contribution of all point sources along the wellbore must be accounted for. Thus, considering that the wellbore section open to flow goes from $y=y_{1}$ to $y=y_{1}+L$ : 


$$
\begin{aligned}
\Delta p_{y}(y, t)=\int_{y_{1}}^{y_{1}+L} \frac{1}{2 \sqrt{\pi \eta_{y} t}} & \exp \left(-\frac{\left(y-y_{1}\right)^{2}}{4 \eta_{y} t}\right) d y= \\
= & \frac{1}{2}\left[\operatorname{erf}\left(\frac{y_{1}+L-y}{2 \sqrt{\eta_{y} t}}\right)-\operatorname{erf}\left(\frac{y_{1}-y}{2 \sqrt{\eta_{y} t}}\right)\right] .
\end{aligned}
$$

Vertically, the reservoir is finite. Therefore, the images method must be applied in $z$-direction [33]. Thus, denoting the reservoir thickness as $h$ and the smallest distance between the wellbore and a vertical boundary as $d z$ :

$$
\Delta p_{z}(z, t)=\frac{1}{2 \sqrt{\pi \eta_{z} t}}\left[\sum_{n=-\infty}^{\infty} \exp \left(-\frac{(2 n h)^{2}}{4 \eta_{z} t}\right)+\exp \left(-\frac{(2 n h-2 d z)^{2}}{4 \eta_{z} t}\right)\right]
$$

Eqs. (A-1) to (A-4) are achieved assuming uniform flux, that is, flow-rate per unit length is constant along the well. However, flow in horizontal wells is more accurately depicted by the infinite conductivity hypothesis, which states that pressure is constant along the wellbore (instead of fluid in flow). Pressure behavior in an infinite conductivity well may be estimated from a uniform influx model, using the average pressure technique or the equivalent pressure point method $[4,21,18,22,34]$.

It is important to notice that Eq. (A-1) does not account for skin effects. In reservoirs with formation damage, an additional term must be included to Eq. (A-1):

$$
\Delta p(x, y, z, t)=\frac{q}{L \phi c_{t}} \int_{0}^{t} \Delta p_{x}(x, \tau) \Delta p_{y}(x, \tau) \Delta p_{z}(x, \tau) d \tau+\frac{q \mu}{L \sqrt{k_{z} k_{x}}} S .
$$

\section{A.2}

\section{Single-Phase Flow in Multilayer Reservoirs}

Some authors have presented analytical solutions for multilateral horizontal or slanted wells $[29,30,28,31]$. Most of these formulations, however, depict pressure behavior in single-layer reservoirs. The model developed by [31], on the other hand, is applicable on both single- and multilayer formations.

The formulation starts by applying Eq. (A-1) into a given layer $j$ :

$$
\Delta p_{j}(x, y, z, t)=\frac{q_{j}}{L_{j} \phi_{j} c_{t_{j}}} \int_{0}^{t} \Delta p_{x_{j}}(x, \tau) \Delta p_{y_{j}}(y, \tau) \Delta p_{z_{j}}(z, \tau) d \tau .
$$

where the directional pressure changes are computed analogously to Eqs. (A-2) 
to $(\mathrm{A}-4)$.

Assuming that layer flow-rate behavior may be considered constant between two consecutive time steps and that the superposition principle holds, then Eq. (A-6) becomes:

$$
\Delta p_{j}\left(t_{i}\right)=\sum_{k=1}^{i} \frac{q_{j}^{k}-q_{j}^{k-1}}{L_{j} \phi_{j} c_{t j}} \int_{t_{k-1}}^{t_{i}} \Delta p_{x j}(x, \tau) \Delta p_{y j}(y, \tau) \Delta p_{z j}(z, \tau) d \tau .
$$

For an infinite conductivity wellbore, pressure is the same in all branches. Moreover, mass balance inside the well states that the sum of each individual lateral flow-rate must equal the total production flow-rate. Thereby, it is possible to build a linear system to determine flow-rate in each well branch:

$$
\left\{\begin{array}{c}
\sum_{j=1}^{n} q_{j}=q \\
p_{j}\left(t_{i}\right)=p_{j+1}\left(t_{i}\right), \quad \forall j=1 \ldots n-1, \forall i
\end{array}\right.
$$

Thus, at each time step, the linear system defined in Eq. (A-8) must be updated and solved, so that flow-rate in each layer is determined. Then, the wellbore pressure behavior may be computed by evaluating the pressure change in any given layer.

It is important to recall that Eqs. (A-6) to (A-8) derive from the formulation proposed by [21] and [18], which assume uniform flux. Therefore, each wellbore branch required to build the linear system defined in Eq. (A-8) is considered to be a uniform flux branch.

In their work, [31] suggested that each well branch could be discretized into several uniform influx segments, to describe more accurately an infinite conductivity well. This, however, increases the linear system order, as more equations are needed. In our work, wellbore pressure change was evaluated using the average pressure technique at each time step, after layer flow-rates have been determined. 


\section{B}

\section{Sketch Proof to Justify the Application of Newman's Product}

Flow throughout the reservoir model depicted in Figs. 3.7 and 3.8 is described by the following PDE:

$$
\frac{\partial^{2} \Delta p_{i}}{\partial y^{2}}+\frac{1}{r} \frac{\partial}{\partial r}\left(r \frac{\partial \Delta p_{i}}{\partial r}\right)-\frac{1}{\eta_{i}} \frac{\partial \Delta p_{i}}{\partial t}=0,
$$

where $i$ indexes the region. Consider for convenience that the flooded zone is already ahead of the damaged region. Then, applying the radially composite model described in Section 3.2, the reservoir may be split into three regions:

Region 1: $r_{w}<r<r_{\text {skin }}$ and $|y|<L / 2$

Region 2: $r_{\text {skin }}<r<r_{F}(t)$ and $|y|<L / 2$

Region 3: $r>r_{F}(t)$ or $|y|>L / 2$

Let $\Delta p_{y}(y, t)$ be the solution of the following one-dimensional PDE:

$$
\frac{\partial^{2} \Delta p}{\partial y^{2}}-\frac{1}{\eta} \frac{\partial \Delta p}{\partial t}=0
$$

Note that $\Delta p_{y}(y, t)$ represents the solution for a one-dimensional flow in $y$-direction, such as represented in Fig. B.1.

Now, let $G_{w}(r, t)$ be the solution of the following one-dimensional PDE:

$$
\frac{1}{r} \frac{\partial}{\partial r}\left(r \frac{\partial \Delta p_{i}}{\partial r}\right)-\frac{1}{\eta_{i}} \frac{\partial \Delta p_{i}}{\partial t}=0 .
$$

$G_{w}(r, t)$ represents the solution for a one-dimensional flow in $r$-direction for a radially heterogeneous reservoir, such as represented in Fig. B.2. By

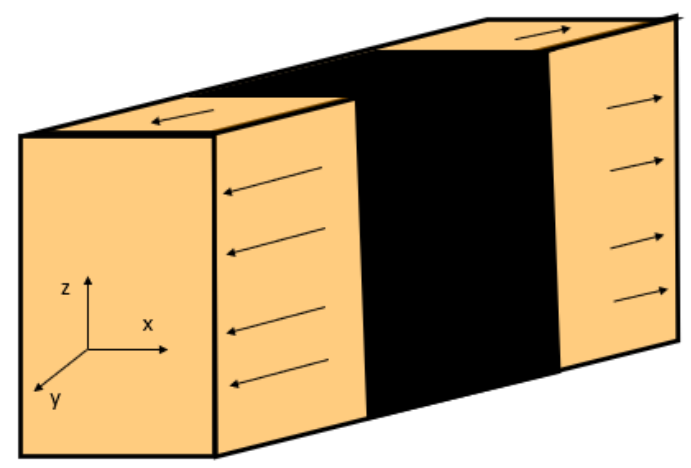

Figure B.1: Schematics of the one-dimensional flow in $y$-direction 


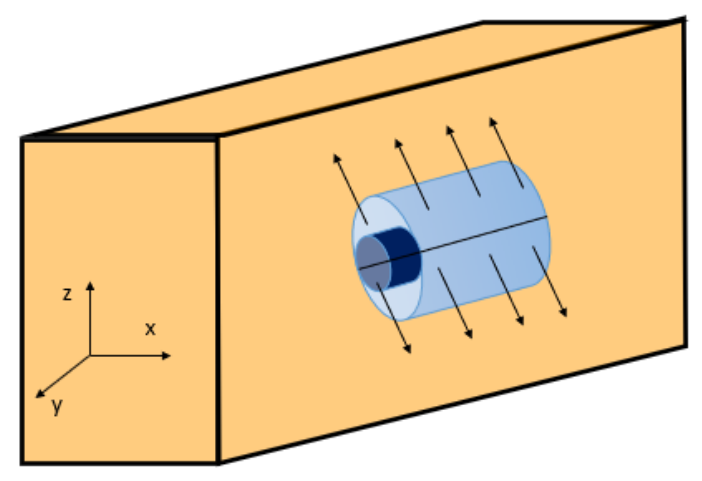

Figure B.2: Schematics of the one-dimensional flow in $r$-direction

construction, the heterogeneities in $r$-direction are already accounted for in the $G_{w}$ function.

Now, define $\Delta p(y, r, t)=\Delta p_{y}(y, t) G_{w}(r, t)$. Then, taking the second derivative of $\Delta p(y, r, t)$ with respect to $y$ :

$$
\frac{\partial^{2} \Delta p(y, r, t)}{\partial y^{2}}=\frac{\partial^{2} \Delta p_{y}(y, t)}{\partial y^{2}} G_{w}(r, t)
$$

Taking the second derivative of $\Delta p(y, r, t)$ with respect to $r$ :

$$
\frac{\partial^{2} \Delta p(y, r, t)}{\partial r^{2}}=\Delta p_{y}(y, t) \frac{\partial^{2} G_{w}(r, t)}{\partial r^{2}}
$$

Taking the derivative of $\Delta p(y, r, t)$ with respect to $t$ :

$$
\frac{\partial \Delta p(y, r, t)}{\partial t}=\frac{\partial \Delta p_{y}(y, t)}{\partial t} G_{w}(r, t)+\Delta p_{y}(y, t) \frac{\partial G_{w}(r, t)}{\partial t}
$$

Then, replacing Eqs. (B-4) to (B-6) into Eq. (B-1):

$$
\frac{\partial^{2} \Delta p_{y}}{\partial y^{2}} G_{w}+\Delta p_{y} \frac{\partial^{2} G_{w}}{\partial r^{2}}-\frac{\partial \Delta p_{y}}{\partial t} G_{w}-\Delta p_{y} \frac{\partial G_{w}}{\partial t}=0
$$

Rearranging Eq. (B-7):

$$
G_{w} \underbrace{\left[\frac{\partial^{2} \Delta p_{y}}{\partial y^{2}}-\frac{\partial \Delta p_{y}}{\partial t}\right]}_{=0 \text {, from Eq. (B-2) }}+\Delta p_{y} \underbrace{\left[\frac{\partial^{2} G_{w}}{\partial r^{2}}-\frac{\partial G_{w}}{\partial t}\right]}_{=0, \text { from Eq. (B-3) }}=0
$$

Therefore, $\Delta p(y, r, t)=\Delta p_{y}(y, t) G_{w}(r, t)$ satisfies the PDE given by Eq. (B-1). The computations made in this Appendix are analogous to the proof presented by Newman [54] and Carslaw and Jaeger [55] to justify the application of Newman's [54] product in homogeneous domains. This Appendix shows that the formulation presented in Section 3.2.3 is valid. However, a more sound proof would still be an interesting topic for further research, specially to verify if Newman's [54] product may also be applied for a general nonhomogeneous domain (or, alternatively, establish the conditions required for Newman's [54] product to be applied in non-homogeneous domains). 